\title{
Predicting the Strength of Stacking Interactions between Hetero-cycles and Aromatic Amino Acid Side Chains
}

\author{
Andrea N. Bootsma, ${ }^{\dagger \S}$ Analise C. Doney, ${ }^{\dagger}$ and Steven E. Wheeler ${ }^{\S *}$ \\ ${ }^{\dagger}$ Department of Chemistry, Texas A\&M University, College Station, TX 77842 \\ ${ }^{\S}$ Computational Quantum Chemistry, Department of Chemistry, University of Georgia, Athens, GA 30602 \\ E-mail: : swheele2@uga.edu
}

Contents

SAPT Analysis of all stacked dimers

$\begin{array}{ll}\text { Figure S1. SAPT0 Energies plot } & \text { S2 }\end{array}$

Other predictive models

$\begin{array}{ll}\text { Figure S2. Fit using equation (4) which includes dipole moment } & \text { S3 }\end{array}$

$\begin{array}{ll}\text { Figure S3. Fit using equation (5) which includes ESP } \text { max }_{\sigma} & \text { S4 }\end{array}$

Figure S4. Fit using equation (6) which include $\mathrm{ESP}_{\sigma} \quad$ S4

Figure S5. Fit using equation (7) which includes $F_{\text {mean }} \quad$ S5

Model Evalutation

Table S1. Correlation coefficients for all fits $\quad$ S6

Figure S6. Predictions from equation (1) split across heterocycle sub-categories S7

Figure S7. Predictions from equation (2) split across heterocycle sub-categories S8

Solution-phase data

Figure S8. Comparison of gas phase vs solution phase geometries $\quad$ S9

Table S2. Solution-phase stacking interaction energies $\quad$ S9

$\begin{array}{ll}\text { Figure S9. Fit for solution phase data } & \text { S10 }\end{array}$

Figure S10. Gas phase vs. solution phase stacking interaction energies $\quad$ S10

Assessment of B97-D

Figure S11. Level of Theory Comparison $\quad$ S11

Other computational data

Table S3. Incremental Focal Point Table for Selected Dimers $\quad$ S12

Table S4. Comparison of Default and Tight PNO cutoffs $\quad$ S13

Table S5. Absolute energies of heterocycles and amino acid side chains $\quad$ S14

Table S6. Absolute Energies, Binding Energies \& SAPT0 Energies Phe $\quad$ S16

Table S7. Absolute Energies, Binding Energies \& SAPT0 Energies Tyr $\quad$ S26

Table S8. Absolute Energies, Binding Energies \& SAPT0 Energies Trp $\quad$ S34

Table S9. Heterocycle Descriptors $\quad$ S47

Table S10. Cross Validation of eqation (1) S51

Table S11. Cross Validation of equation (2) $\quad$ S54

Table S12. Test Set Absolute Energies \& Binding Energies Phe $\quad$ S57

Table S13. Test Set Absolute Energies \& Binding Energies Tyr $\quad$ S60

Table S14. Test Set Absolute Energies \& Binding Energies Trp $\quad$ S62 


\section{SAPT Analysis of all stacked dimers}

SAPT enables the decomposition of intermolecular interaction energies into electrostatic $\left(\mathrm{E}_{\text {elec }}\right)$, dispersion $\left(E_{\text {disp }}\right)$, exchange-repulsion $\left(E_{\text {exch }}\right)$, and induction ( $\left.E_{\text {ind }}\right)$ components, and can be used to identify the effects that drive the trend in interaction energies. Considering all stacked local energy minima for Phe, Tyr, and Trp, the lack of correlation between the total SAPT0 interaction energy $\left(E_{\mathrm{SAPT} 0}\right)$ and both the non-electrostatic component $\left(E_{\text {non-elec }}=E_{\text {disp }}+E_{\text {ind }}+E_{\text {exch }}, R^{2}=\right.$ $0.08)$ and the non-dispersion component $\left(E_{\text {non-disp }}=E_{\text {elec }}+E_{\text {ind }}+E_{\text {exch }}, R^{2}=0.03\right)$ indicates that electrostatic and dispersion interactions are vital to capture the overall trends. Moreover, they are of similar importance in determining how strongly a given heterocycle will stack with these aromatic amino acid side chains.

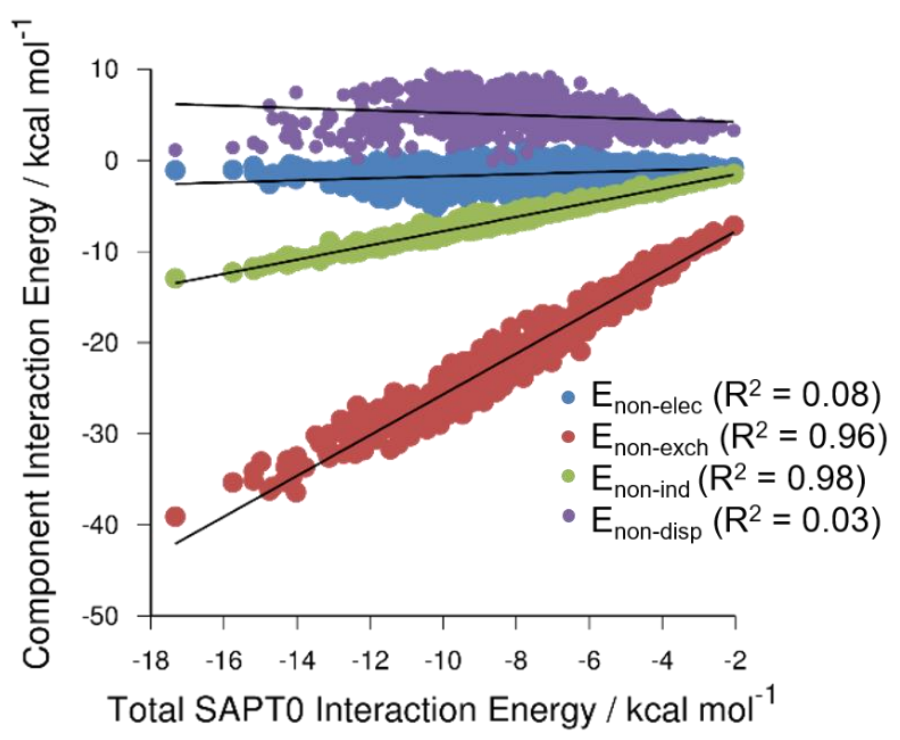

Figure S1. Non-electrostatic, non-exchange, non-induction, and non-dipersion components versus the total SAPT0 energies for all energy minima. 


\section{Other Predictive Models}

In addition to equations (1) and (2) presented in the main text, we also considered other combinations of molecular descriptors that gave rise to fits of similar quality and robustness. Below are plots of four of these fits (equations 3-6 below). These primarily vary in the way that the electrostatic inhomogeneity is captured, which is described by $\mathrm{ESP}_{\text {range }}$ in equation (1). These alternate modes of describing electrostatic variation in the heterocycle include dipole moment $(\mu)$, maximum electrostatic potential $\left(\mathrm{ESP}_{\max }\right)$, standard deviation of the electrostatic potential $\left(\mathrm{ESP}_{\sigma}\right)$, and average electric field $\left(\mathrm{F}_{\text {mean }}\right)$.

$\Delta \mathrm{E}_{\text {pred }}=\mathrm{N}_{\mathrm{HA}}^{\mathrm{AA}}\left(-0.03 \mu-0.033 \mathrm{ESP}_{\text {mean }}^{\text {Het }}-0.099 \mathrm{~N}_{\mathrm{HA}}^{\mathrm{Het}}\right)-1.75$
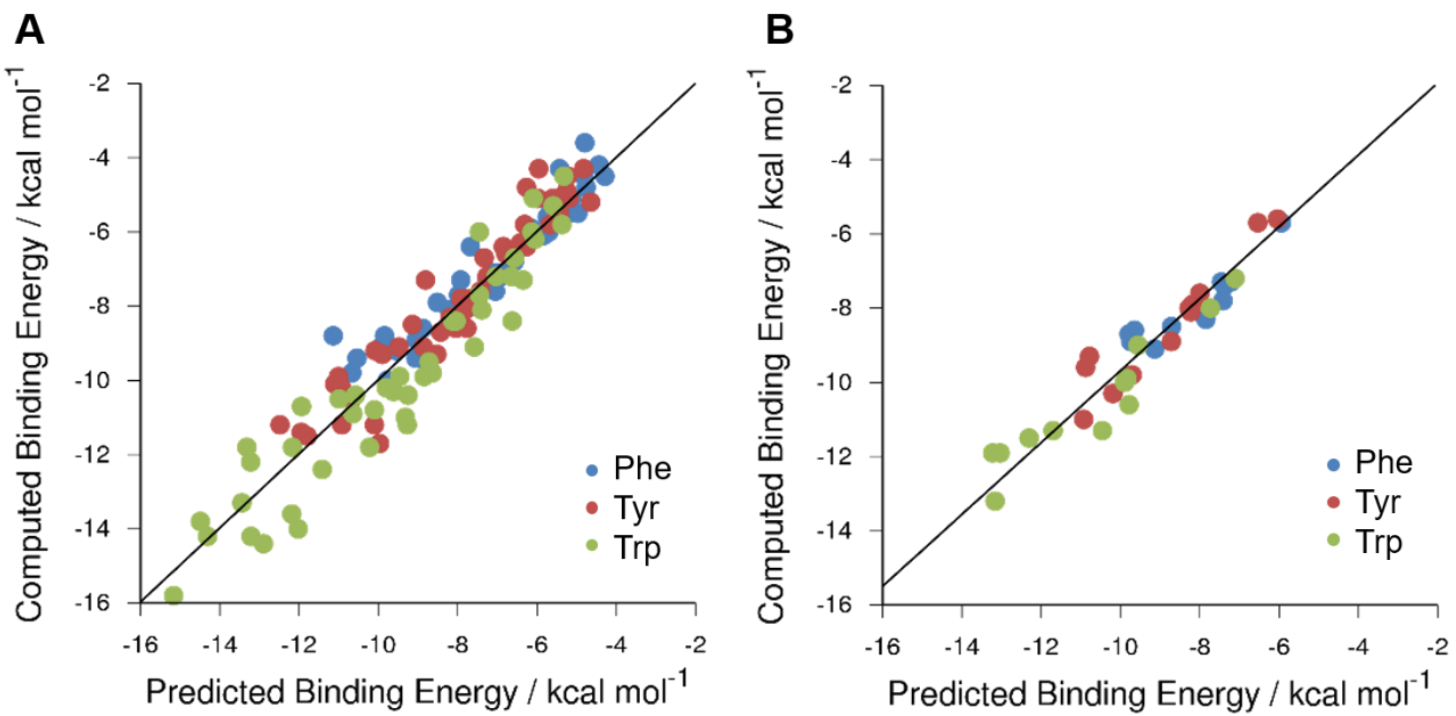

Figure S2. Scatter plot of computed DLPNO-CCSD(T)/cc-pVQZ interaction energies vs predicted interaction energies from equation (4) for global minimum energy stacked dimers of $\mathrm{A}$. training set heterocycles 1-46 and B. test set heterocycles 47-58 with Phe, Tyr, and Trp side chains. 


$$
\Delta \mathrm{E}_{\text {pred }}=\mathrm{N}_{\mathrm{HA}}^{\mathrm{AA}}\left(-0.022 \mathrm{ESP}_{\max }^{\mathrm{Het}}-0.022 \mathrm{ESP}_{\text {mean }}^{\mathrm{Het}}-0.095 \mathrm{~N}_{\mathrm{HA}}^{\mathrm{Het}}\right)-1.67
$$
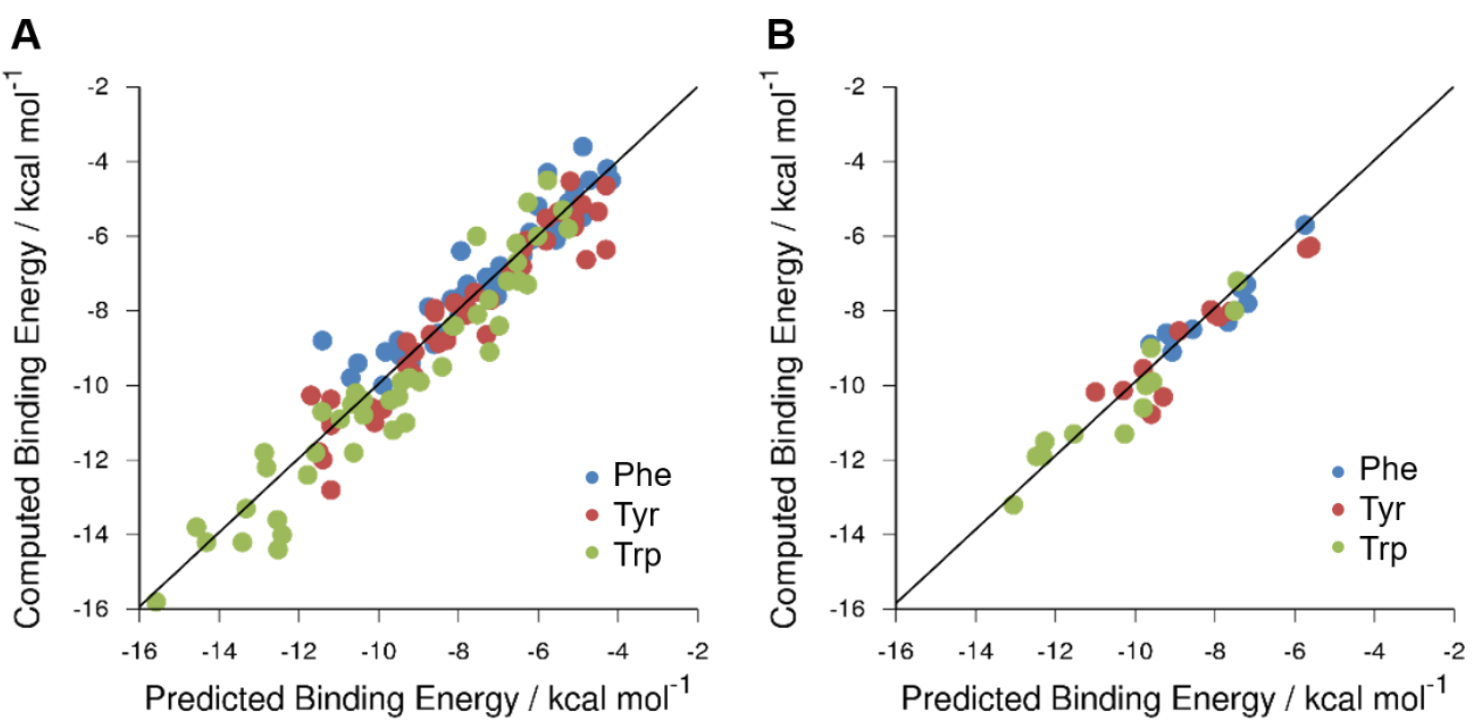

Figure S3. Scatter plot of computed DLPNO-CCSD(T)/cc-pVQZ interaction energies vs predicted interaction energies from equation (5) for global minimum energy stacked dimers of A. training set heterocycles 1-46 and B. test set heterocycles 47-58 with Phe, Tyr, and Trp side chains.

$\Delta \mathrm{E}_{\text {pred }}=\mathrm{N}_{\mathrm{HA}}^{\mathrm{AA}}\left(-0.047 \mathrm{ESP}_{\sigma}^{\mathrm{Het}}-0.034 \mathrm{ESP}_{\text {mean }}^{\mathrm{Het}}-0.096 \mathrm{~N}_{\mathrm{HA}}^{\mathrm{Het}}\right)-1.28$
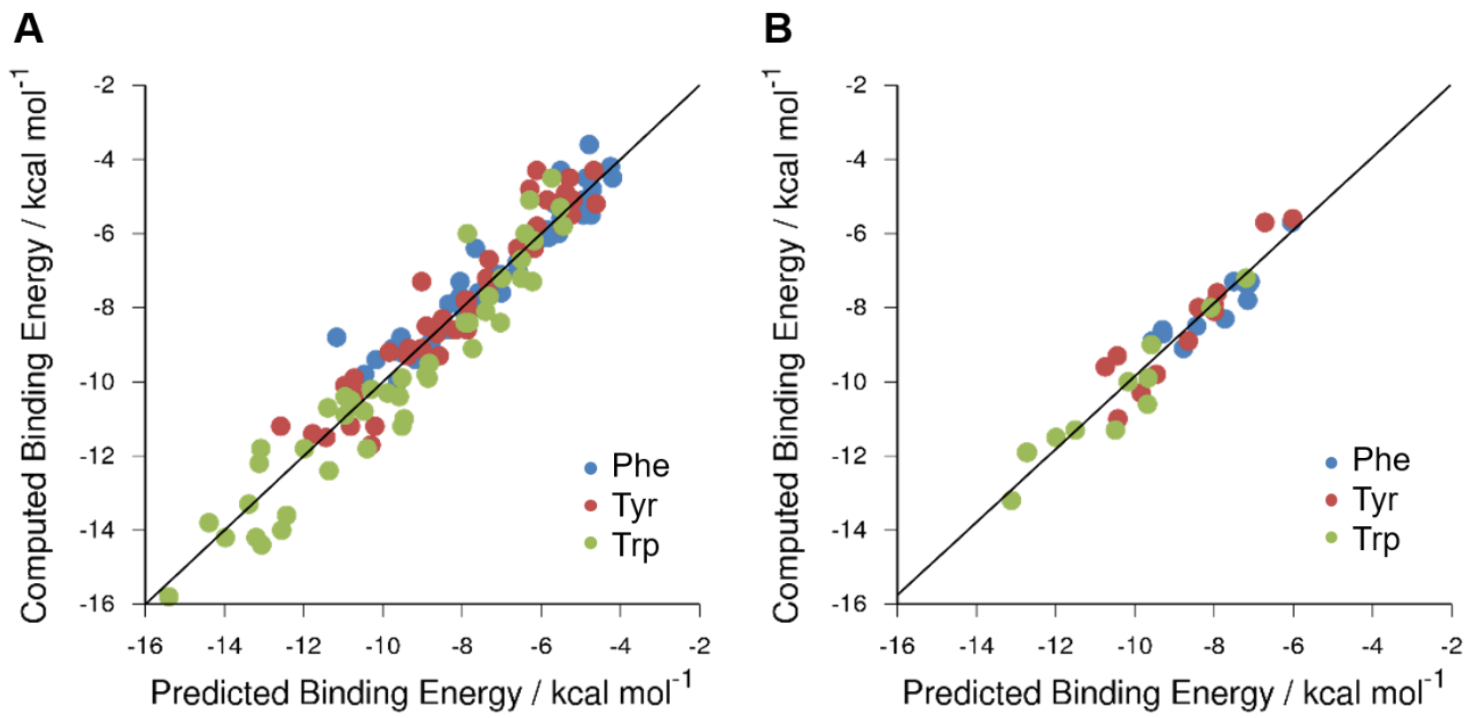

Figure S4. Scatter plot of computed DLPNO-CCSD(T)/cc-pVQZ interaction energies vs predicted interaction energies from equation (6) for global minimum energy stacked dimers of A. training set heterocycles 1-46 and B. test set heterocycles 47-58 with Phe, Tyr, and Trp side chains. 


$$
\Delta \mathrm{E}_{\text {pred }}=\mathrm{N}_{\mathrm{HA}}^{\mathrm{AA}}\left(-0.46 \mathrm{~F}_{\text {mean }}^{\mathrm{Het}}-0.042 \mathrm{ESP}_{\text {mean }}^{\mathrm{Het}}-0.098 \mathrm{~N}_{\mathrm{HA}}^{\mathrm{Het}}\right)-0.67
$$
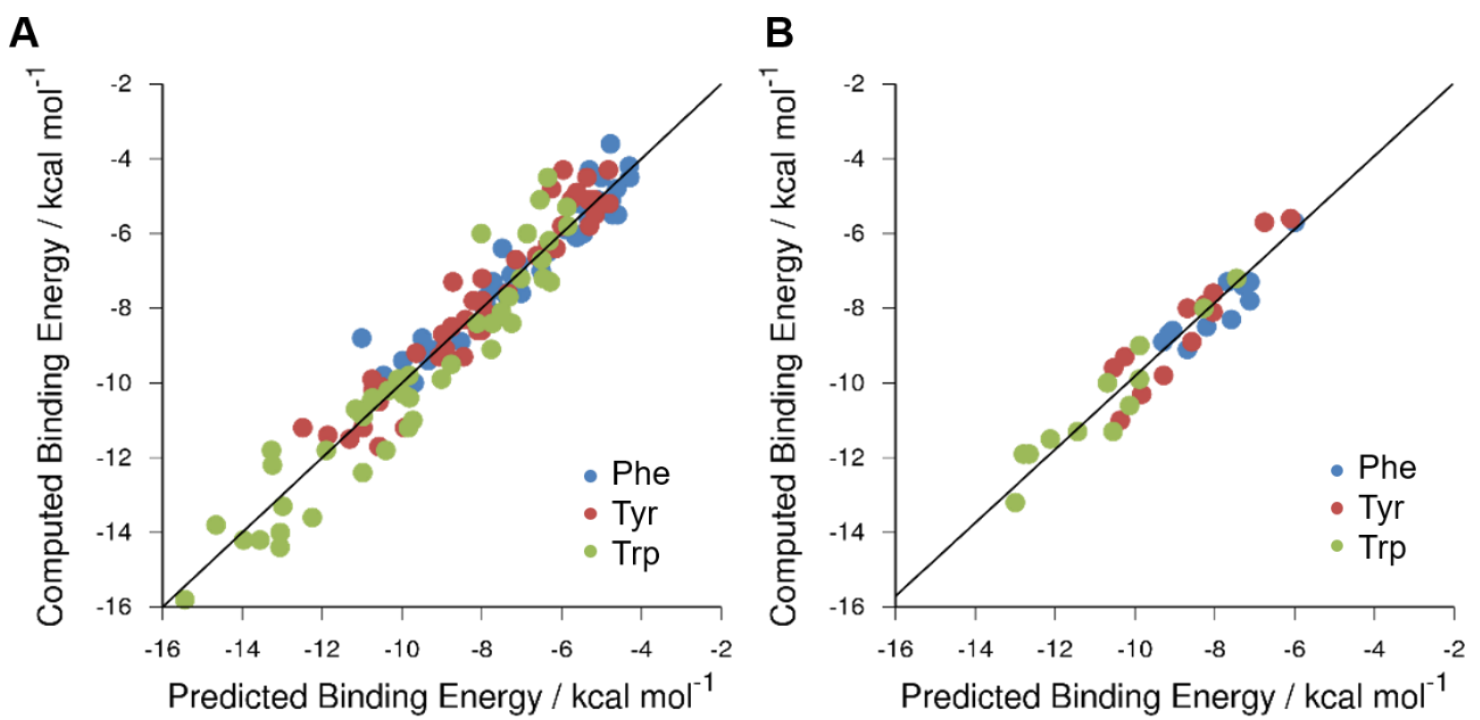

Figure S5. Scatter plot of computed DLPNO-CCSD(T)/cc-pVQZ interaction energies vs predicted interaction energies from equation (7) for global minimum energy stacked dimers of A. training set heterocycles 1-46 and B. test set heterocycles 47-58 with Phe, Tyr, and Trp side chains. 
Model Evaluation

Table S1. Correlations between computed and predicted interaction energies for equations (1)(6)

\begin{tabular}{|c|c|c|c|c|c|c|c|}
\hline & & $\begin{array}{c}\text { Equation } \\
\text { (1) }\end{array}$ & $\begin{array}{c}\text { Equation } \\
\text { (2) }\end{array}$ & $\begin{array}{c}\text { Equation } \\
\text { (3) }\end{array}$ & $\begin{array}{c}\text { Equation } \\
\text { (4) }\end{array}$ & $\begin{array}{c}\text { Equation } \\
(5)\end{array}$ & $\begin{array}{c}\text { Equation } \\
\text { (6) }\end{array}$ \\
\hline \multirow{8}{*}{$\begin{array}{c}\text { Training Set } \\
(1-46)\end{array}$} & $\mathbf{R}^{2}$ & 0.92 & 0.90 & 0.91 & 0.91 & 0.92 & 0.92 \\
\hline & $\mathbf{Q}^{2}$ & 0.92 & 0.89 & 0.9 & 0.91 & 0.91 & 0.92 \\
\hline & $\mathbf{R}^{2}{ }_{10 \text {-fold }}$ & 0.91 & 0.89 & 0.89 & 0.9 & 0.91 & 0.91 \\
\hline & $\mathbf{R}^{2}$ 5-fold & 0.90 & 0.88 & 0.89 & 0.89 & 0.90 & 0.9 \\
\hline & $\mathbf{R}^{2}{ }_{\text {3-fold }}$ & 0.89 & 0.87 & 0.88 & 0.88 & 0.89 & 0.90 \\
\hline & $\rho^{2}$ & 0.92 & 0.90 & 0.92 & 0.91 & 0.92 & 0.92 \\
\hline & $\tau$ & 0.84 & 0.81 & 0.83 & 0.82 & 0.83 & 0.84 \\
\hline & RMSE & 0.7 & 0.8 & 0.8 & 0.8 & 0.7 & 0.7 \\
\hline \multirow{4}{*}{$\begin{array}{c}\text { Test Set (47- } \\
58)\end{array}$} & $\mathbf{R}^{2}$ & 0.91 & 0.92 & 0.91 & 0.92 & 0.91 & 0.91 \\
\hline & $\rho^{2}$ & 0.91 & 0.90 & 0.93 & 0.91 & 0.93 & 0.91 \\
\hline & $\tau$ & 0.84 & 0.83 & 0.86 & 0.84 & 0.86 & 0.84 \\
\hline & RMSE & 0.6 & 0.5 & 0.6 & 0.5 & 0.6 & 0.6 \\
\hline
\end{tabular}


Figure S6. Scatter plot of computed DLPNO-CCSD(T)/cc-pVQZ interaction energies vs predicted interaction energies from equation (1) for global minimum energy stacked dimers of the training set (1-46) split into sub categories, and with each amino acid shown separately

Phe
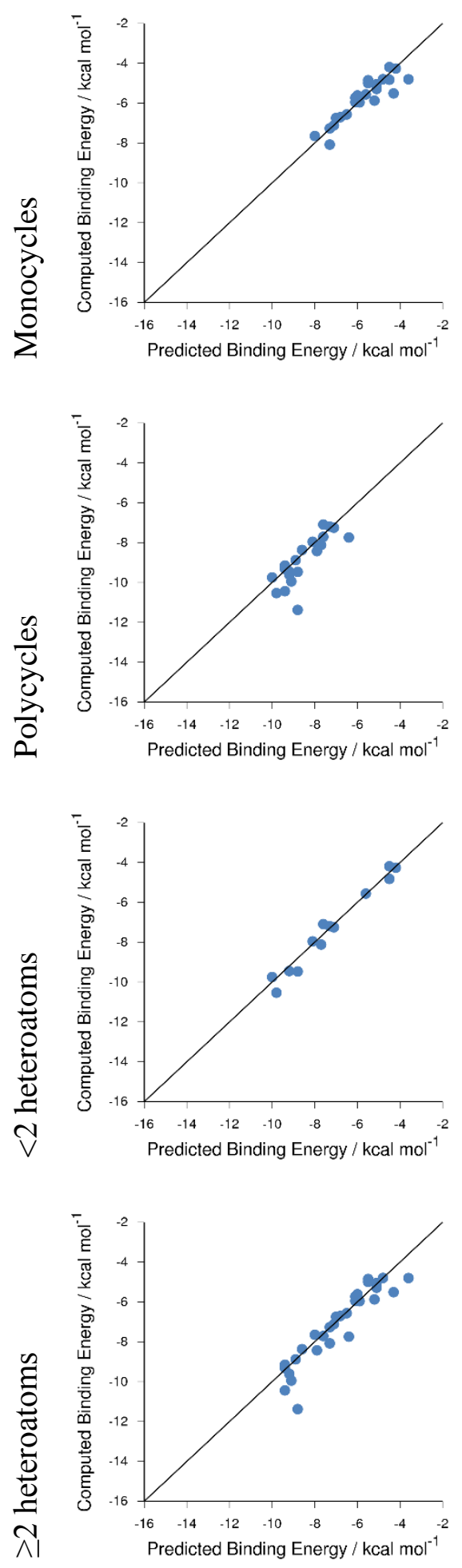

Tyr
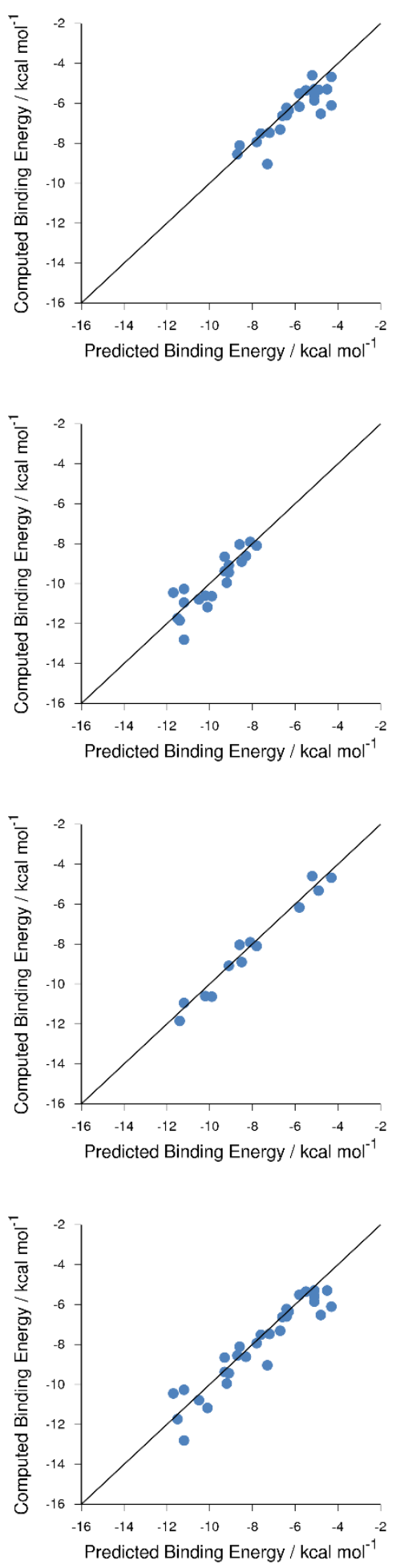

$\operatorname{Trp}$
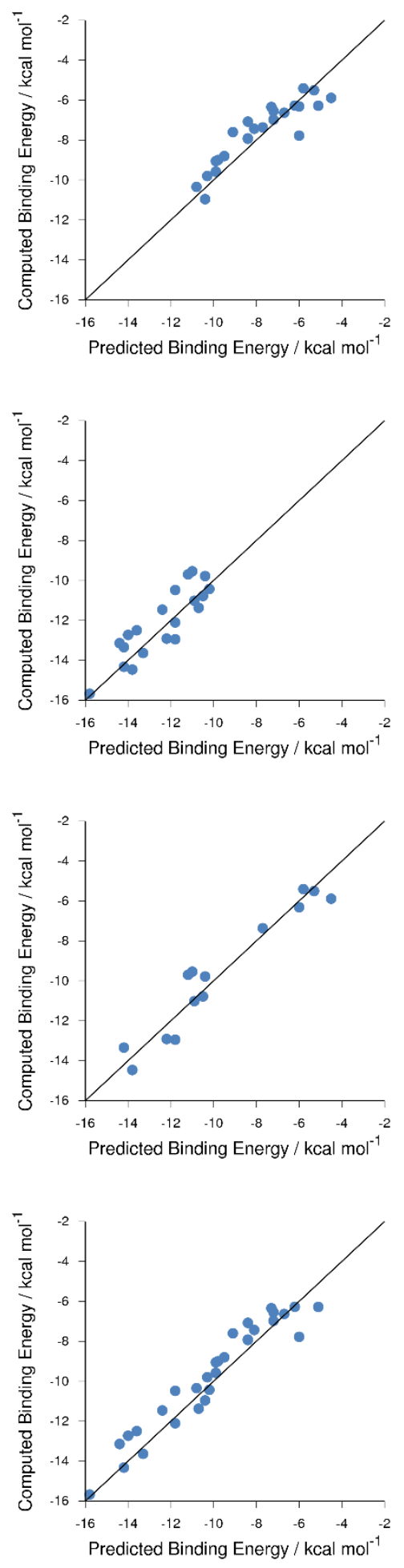
Figure S7. Scatter plot of computed DLPNO-CCSD(T)/cc-pVQZ interaction energies vs predicted interaction energies from equation (2) for global minimum energy stacked dimers of the training set (1-46) split into sub categories, and with each amino acid shown separately

Phe
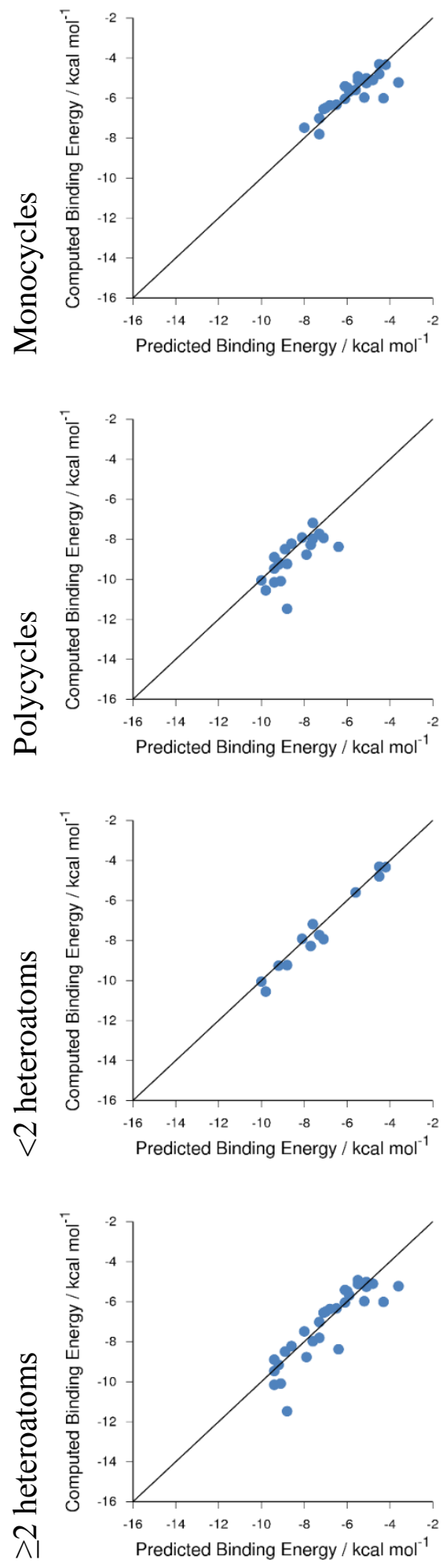

Tyr
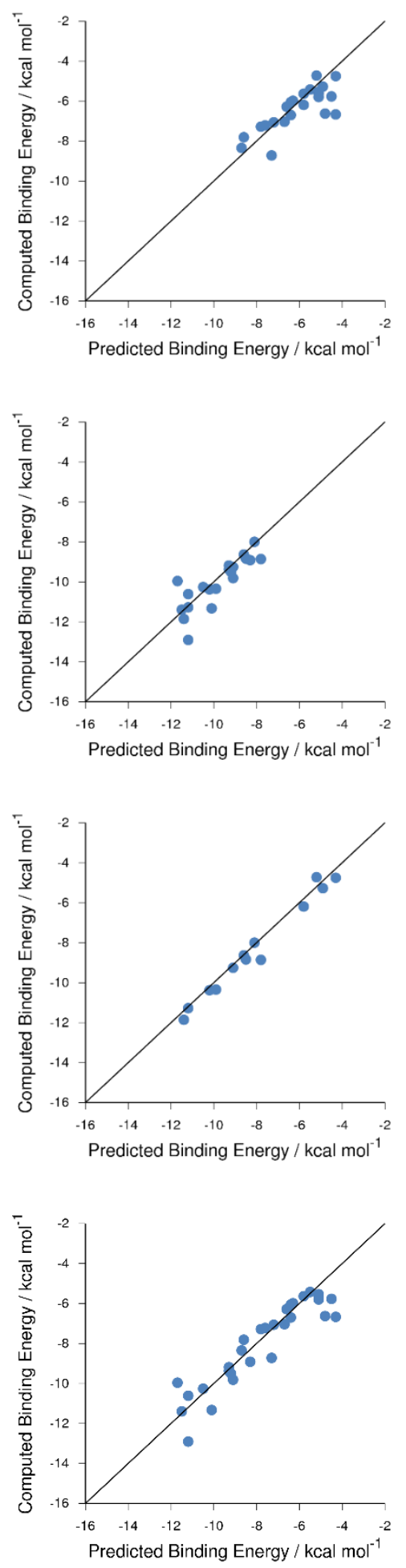

$\operatorname{Trp}$
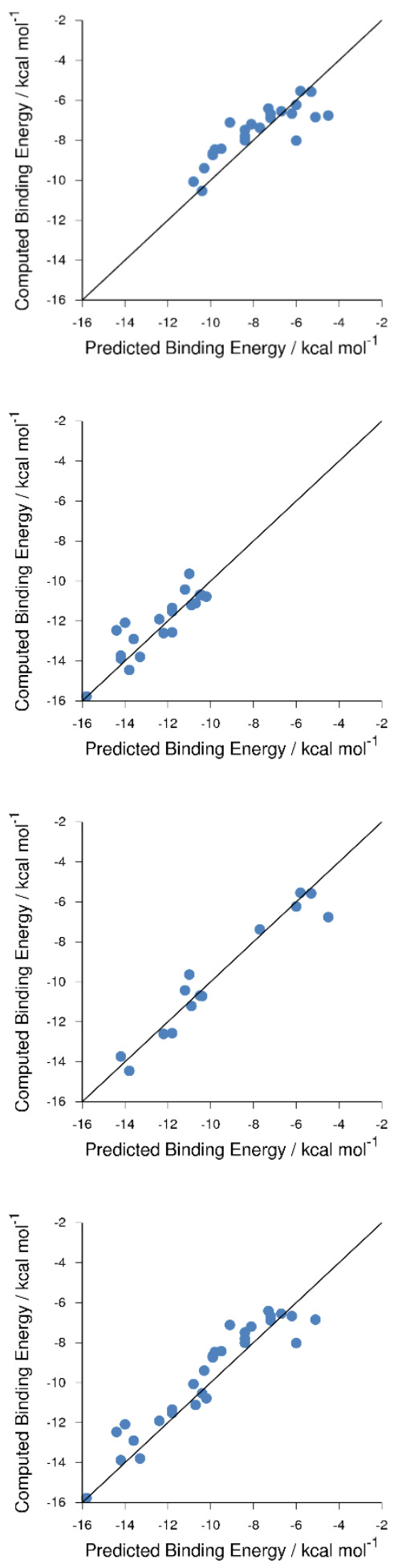


\section{Solution-Phase Data}
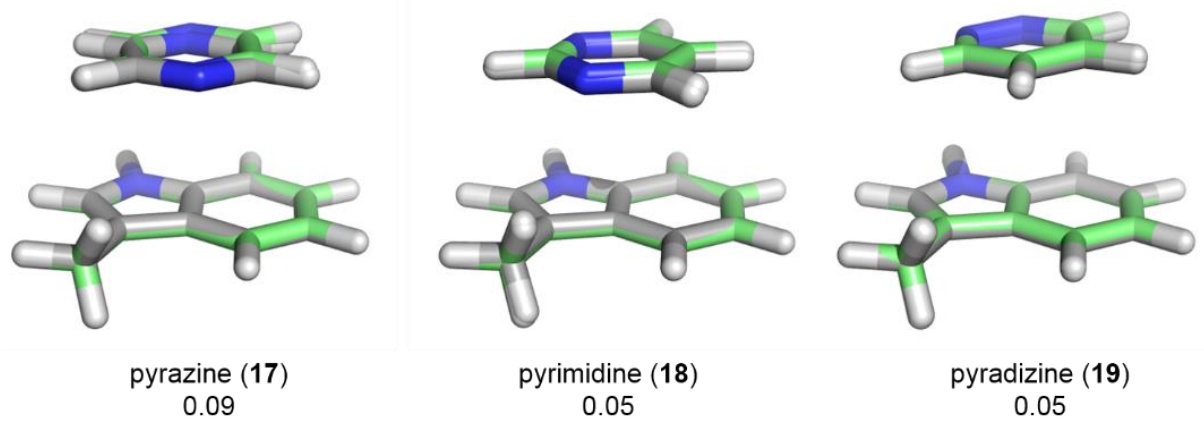

Figure S8. Superimposed gas-phase (grey) and solution-phase (green) structures for pyrazine (17), pyrimidine (18), and pyridazine (19) stacked with Trp, along with heavy-atom RMSD values in Angstroms.

Table S2. Solution-phase (diethyl ether) interaction energies of the global minimum stacked dimers of 1-46 with Phe, Tyr, and Trp.

\begin{tabular}{cccccccc}
\hline Het & $\boldsymbol{E}_{\text {int }}^{\boldsymbol{P h} \boldsymbol{e}}$ & $\boldsymbol{E}_{\boldsymbol{i n t}}^{\boldsymbol{T y r}}$ & $\boldsymbol{E}_{\text {int }}^{\boldsymbol{T r p}}$ & $\mathbf{H e t}$ & $\boldsymbol{E}_{\text {int }}^{\boldsymbol{P h e}}$ & $\boldsymbol{E}_{\text {int }}^{\boldsymbol{T y r}}$ & $\boldsymbol{E}_{\boldsymbol{i n t}}^{\boldsymbol{T r p}}$ \\
\hline $\mathbf{1}$ & - & - & -1.7 & $\mathbf{2 4}$ & -3.0 & -2.8 & -4.5 \\
$\mathbf{2}$ & -2.2 & -2.0 & -2.9 & $\mathbf{2 5}$ & -4.6 & -4.5 & -6.1 \\
$\mathbf{3}$ & -2.9 & -2.9 & -3.5 & $\mathbf{2 6}$ & -2.9 & -2.8 & -4.1 \\
$\mathbf{4}$ & -2.5 & -2.4 & -3.4 & $\mathbf{2 7}$ & -3.8 & -4.0 & -5.0 \\
$\mathbf{5}$ & -2.7 & -2.5 & -3.6 & $\mathbf{2 8}$ & -3.6 & -3.7 & -4.8 \\
$\mathbf{6}$ & -3.2 & -3.2 & -3.8 & $\mathbf{2 9}$ & -4.2 & -4.1 & -5.5 \\
$\mathbf{7}$ & -3.2 & -3.0 & -4.2 & $\mathbf{3 0}$ & -3.2 & -4.4 & -4.8 \\
$\mathbf{8}$ & -1.8 & -2.3 & -2.3 & $\mathbf{3 1}$ & -3.9 & -4.4 & -5.3 \\
$\mathbf{9}$ & - & - & -3.0 & $\mathbf{3 2}$ & -4.5 & -4.6 & -5.7 \\
$\mathbf{1 0}$ & -1.1 & -0.8 & - & $\mathbf{3 3}$ & -4.0 & -4.1 & -5.0 \\
$\mathbf{1 1}$ & - & - & -2.7 & $\mathbf{3 4}$ & -4.1 & -4.6 & -5.7 \\
$\mathbf{1 2}$ & -2.5 & -2.5 & -3.0 & $\mathbf{3 5}$ & -4.8 & -4.8 & -6.8 \\
$\mathbf{1 3}$ & -2.1 & -1.3 & - & $\mathbf{3 6}$ & -4.1 & -4.1 & -5.3 \\
$\mathbf{1 4}$ & -3.5 & -3.7 & -5.4 & $\mathbf{3 7}$ & -4.6 & -4.9 & -6.7 \\
$\mathbf{1 5}$ & -2.5 & -2.4 & -3.0 & $\mathbf{3 8}$ & -4.8 & -4.7 & -5.8 \\
$\mathbf{1 6}$ & -3.0 & -3.0 & -4.0 & $\mathbf{3 9}$ & -4.7 & -4.7 & -5.9 \\
$\mathbf{1 7}$ & -3.5 & -3.5 & -4.7 & $\mathbf{4 0}$ & -4.7 & -4.7 & -5.6 \\
$\mathbf{1 8}$ & -3.0 & -3.1 & -4.0 & $\mathbf{4 1}$ & -4.8 & -5.3 & -6.2 \\
$\mathbf{1 9}$ & -3.8 & -3.4 & -5.0 & $\mathbf{4 2}$ & -5.0 & -5.2 & -6.6 \\
$\mathbf{2 0}$ & -1.7 & -1.9 & -2.9 & $\mathbf{4 3}$ & -4.9 & -5.6 & -7.1 \\
$\mathbf{2 1}$ & -3.8 & -3.8 & -4.9 & $\mathbf{4 4}$ & -5.1 & -5.4 & -6.3 \\
$\mathbf{2 2}$ & -4.0 & -4.4 & -5.6 & $\mathbf{4 5}$ & -5.5 & -5.9 & -6.6 \\
$\mathbf{2 3}$ & -2.8 & -2.8 & -4.4 & $\mathbf{4 6}$ & -6.6 & -7.4 & -8.6 \\
\hline & & & & & & &
\end{tabular}




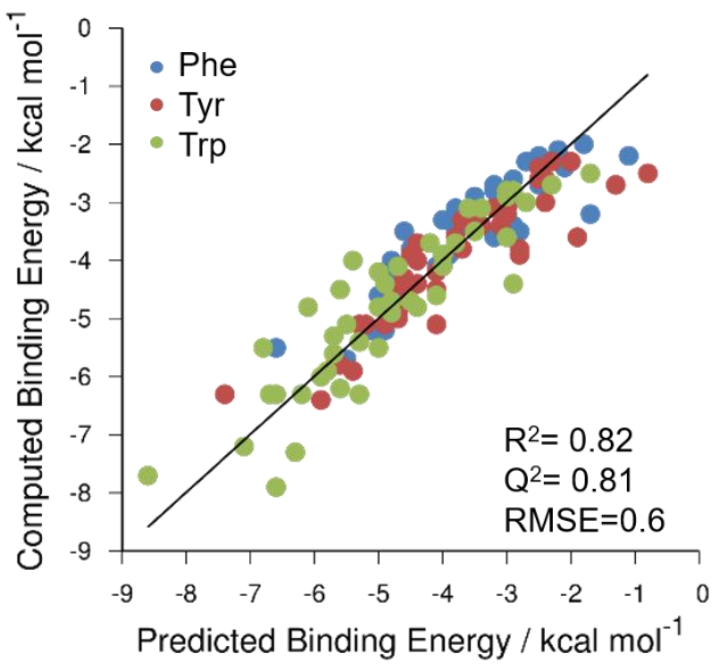

Figure S9. Correlation of computed interaction energies in solution $(\varepsilon=4.24)$ with predicted interaction energies from equation (3) from the main text for the global minimum energy stacked dimers for heterocycles 1-46.

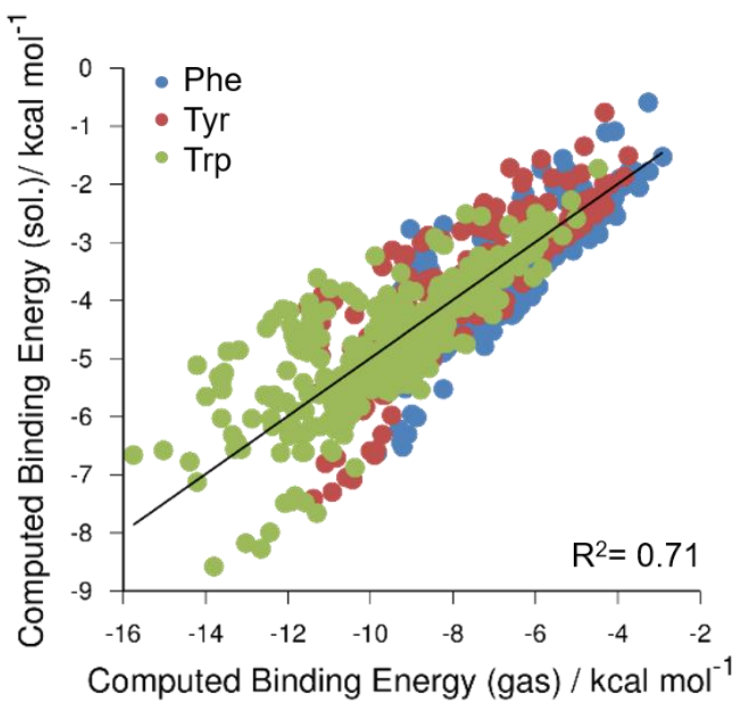

Figure S10. Comparison of interaction energies computed for all stacked local minima in both the gas phase and the solution phase $(\varepsilon=4.24)$. 


\section{Assessment of B97-D}

To assess the performance of B97-D/def2TZVPP (which was used to optimize dimer geometries) for these stacking interactions, we have compared the B97-D interaction energies with the DLPNO-CCSD(T)/cc-pVQZ data. While the DFT-computed interaction energies are strongly correlated with the DLPNO-CCSD $(\mathrm{T})$ data, there are systematic errors when considering stacking with different amino acid side chains (see Figure S2).

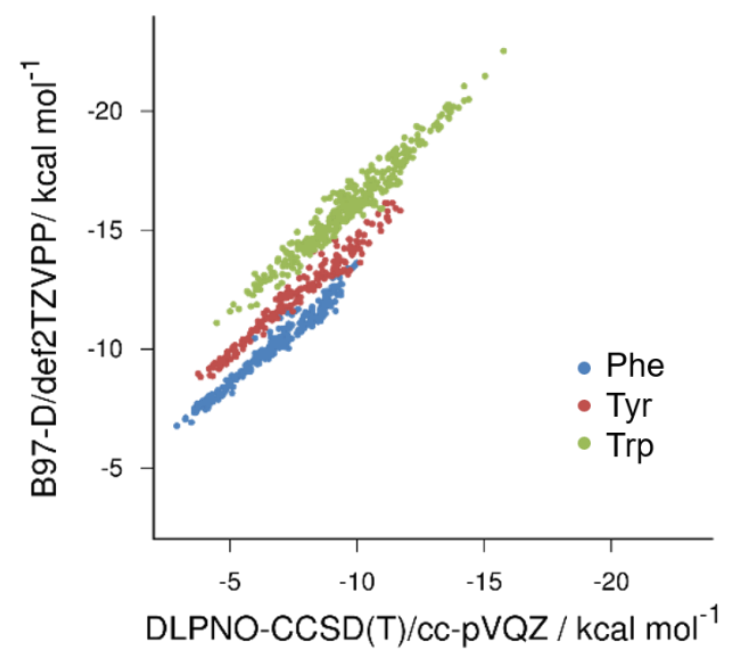

Figure S11. Comparison of interaction energies computed using B97-D/def2TZVPP and DLPNO-CCSD(T)/cc-pVQZ for all stacked minima 
Other Computational Data

Table S3. Incremental focal point tables for selected stacked dimers (relative energies in kcal $\mathrm{mol}^{-1}$ ). Extrapolated complete basis set (CBS) limit values are in brackets.

\begin{tabular}{|c|c|c|c|c|c|}
\hline & HF & $+\delta S L M P 2$ & $\begin{array}{l}+\delta \text { DLPNO- } \\
\text { CCSD }\end{array}$ & $\begin{array}{l}+\delta \text { DLPNO- } \\
\text { CCSD(T) }\end{array}$ & $\begin{array}{l}=\text { DLPNO- } \\
\text { CCSD }(T)\end{array}$ \\
\hline & \multicolumn{5}{|c|}{ Phe'*benzene } \\
\hline cc-pVDZ & 4.18 & -10.16 & -0.05 & -1.1 & {$[-7.13]$} \\
\hline cc-pVTZ & 6.33 & -10.28 & 0.36 & -1.4 & {$[-5.00]$} \\
\hline cc-pVQZ & 6.7 & -10.18 & 0.49 & -1.5 & {$[-4.48]$} \\
\hline \multirow[t]{2}{*}{ CBS LIMIT } & {$[+6.76]$} & {$[-10.10]$} & {$[+0.58]$} & {$[-1.56]$} & {$[-4.33]$} \\
\hline & \multicolumn{5}{|c|}{ Phe $\cdots$ pyrazole } \\
\hline cc-pVDZ & 4.38 & -9.35 & 0 & -1.05 & {$[-6.02]$} \\
\hline cc-pVTZ & 6.22 & -9.41 & 0.37 & -1.31 & {$[-4.13]$} \\
\hline cc-pVQZ & 6.64 & -9.32 & 0.52 & -1.42 & {$[-3.59]$} \\
\hline \multirow[t]{2}{*}{ CBS LIMIT } & {$[+6.75]$} & {$[-9.26]$} & {$[+0.63]$} & {$[-1.50]$} & {$[-3.38]$} \\
\hline & \multicolumn{5}{|c|}{ Phe $\cdots 3,9-d i h y d r o-p u r i n e-2,6-d i o n e$} \\
\hline cc-pVDZ & 2.76 & -12.82 & 0.1 & -0.84 & {$[-10.80]$} \\
\hline cc-pVTZ & 5.06 & -14.13 & 0.2 & -1.14 & {$[-10.00]$} \\
\hline cc-pVQZ & 5.74 & -14.27 & 0.37 & -1.23 & {$[-9.40]$} \\
\hline \multirow[t]{2}{*}{ CBS LIMIT } & {$[+6.02]$} & {$[-14.38]$} & {$[+0.49]$} & {$[-1.31]$} & {$[-9.18]$} \\
\hline & \multicolumn{5}{|c|}{ Phe $\cdots$ indolizine } \\
\hline cc-pVDZ & 3.77 & -11.65 & 0.28 & -0.83 & {$[-8.43]$} \\
\hline cc-pVTZ & 5.46 & -12.88 & 0.62 & -1.1 & {$[-7.90]$} \\
\hline cc-pVQZ & 5.91 & -12.88 & 0.55 & -1.23 & {$[-7.65]$} \\
\hline \multirow[t]{2}{*}{ CBS LIMIT } & {$[+6.07]$} & {$[-12.88]$} & {$[+0.49]$} & {$[-1.33]$} & {$[-7.64]$} \\
\hline & \multicolumn{5}{|c|}{ Tyr'benzene } \\
\hline cc-pVDZ & 3.99 & -10.85 & 0.07 & -1.15 & {$[-7.95]$} \\
\hline cc-pVTZ & 6.37 & -11.09 & 0.49 & -1.46 & {$[-5.69]$} \\
\hline cc-pVQZ & 6.88 & -10.91 & 0.66 & -1.55 & {$[-4.92]$} \\
\hline \multirow[t]{2}{*}{ CBS LIMIT } & {$[+7.02]$} & {$[-10.78]$} & {$[+0.77]$} & {$[-1.61]$} & {$[-4.61]$} \\
\hline & \multicolumn{5}{|c|}{ Tyr'pyrazole } \\
\hline cc-pVDZ & 4.27 & -10.48 & 0.09 & -1.16 & {$[-7.28]$} \\
\hline cc-pVTZ & 6.45 & -10.65 & 0.46 & -1.44 & {$[-5.18]$} \\
\hline cc-pVQZ & 6.96 & -10.54 & 0.59 & -1.56 & {$[-4.54]$} \\
\hline \multirow[t]{2}{*}{ CBS LIMIT } & {$[+7.11]$} & {$[-10.45]$} & {$[+0.69]$} & {$[-1.64]$} & {$[-4.29]$} \\
\hline & \multicolumn{5}{|c|}{ Tyr'*3,9-dihydro-purine-2,6-dione } \\
\hline cc-pVDZ & 0.78 & -13.24 & -0.12 & -0.92 & {$[-13.50]$} \\
\hline cc-pVTZ & 3.48 & -14.45 & -0.21 & -1.17 & {$[-12.34]$} \\
\hline cc-pVQZ & 4.36 & -14.52 & -0.1 & -1.25 & {$[-11.51]$} \\
\hline \multirow[t]{2}{*}{ CBS LIMIT } & {$[+4.75]$} & {$[-14.56]$} & {$[-0.03]$} & {$[-1.31]$} & {$[-11.16]$} \\
\hline & \multicolumn{5}{|c|}{ Tyr $\cdots$ indolizine } \\
\hline cc-pVDZ & 4.05 & -13.24 & 0.72 & -0.99 & {$[-9.45]$} \\
\hline cc-pVTZ & 6.06 & -14.44 & 0.98 & -1.34 & {$[-8.72]$} \\
\hline cc-pVQZ & 6.69 & -14.43 & 1.09 & -1.45 & {$[-8.10]$} \\
\hline \multirow[t]{2}{*}{ CBS LIMIT } & [+6.95] & {$[-14.43]$} & [+1.17] & {$[-1.54]$} & {$[-7.85]$} \\
\hline & \multicolumn{5}{|c|}{ Trp*benzene } \\
\hline cc-pVDZ & 5.37 & -13.42 & 0.18 & -1.43 & {$[-9.30]$} \\
\hline cc-pVTZ & 7.9 & -13.48 & 0.62 & -1.73 & {$[-6.70]$} \\
\hline cc-pVQZ & 8.34 & -13.3 & 0.8 & -1.86 & {$[-6.03]$} \\
\hline CBS LIMIT & {$[+8.40]$} & {$[-13.17]$} & {$[+0.93]$} & {$[-1.96]$} & {$[-5.80]$} \\
\hline
\end{tabular}




\begin{tabular}{|c|c|c|c|c|c|}
\hline & \multicolumn{5}{|c|}{ Trp $\cdots$ pyrazole } \\
\hline cc-pVDZ & 4.17 & -11.16 & -0.14 & -1.34 & {$[-8.47]$} \\
\hline cc-pVTZ & 6.48 & -11.14 & 0.22 & -1.56 & {$[-6.00]$} \\
\hline cc-pVQZ & 6.98 & -10.9 & 0.26 & -1.65 & {$[-5.31]$} \\
\hline \multirow[t]{2}{*}{ CBS LIMIT } & {$[+7.12]$} & {$[-10.73]$} & {$[+0.29]$} & {$[-1.72]$} & {$[-5.04]$} \\
\hline & \multicolumn{5}{|c|}{ Trp $\cdots 3,9-d i h y d r o-p u r i n e-2,6-d i o n e$} \\
\hline cc-pVDZ & 0.6 & -16.52 & 0.81 & -1.34 & {$[-16.46]$} \\
\hline cc-pVTZ & 3.71 & -18.11 & 0.95 & -1.67 & {$[-15.12]$} \\
\hline cc-pVQZ & 4.7 & -18.19 & 1.08 & -1.8 & {$[-14.21]$} \\
\hline \multirow[t]{2}{*}{ CBS LIMIT } & {$[+5.13]$} & {$[-18.24]$} & {$[+1.17]$} & {$[-1.89]$} & {$[-13.83]$} \\
\hline & \multicolumn{5}{|c|}{ Trp $\cdots$ indolizine } \\
\hline cc-pVDZ & 3.1 & -14.88 & 0.25 & -1.21 & {$[-12.74]$} \\
\hline cc-pVTZ & 5.51 & -16.15 & 0.51 & -1.52 & {$[-11.66]$} \\
\hline cc-pVQZ & 7.8 & -17.02 & 1.47 & -1.87 & {$[-9.62]$} \\
\hline CBS LIMIT & {$[+9.29]$} & {$[-17.66]$} & {$[+2.17]$} & {$[-2.12]$} & {$[-8.31]$} \\
\hline
\end{tabular}

Table S4. Comparison of DLPNO-CCSD(T)/cc-pVQZ interaction energies using both the default and tight PNO cutoffs, while the Default PNO cutoff (which is used in the analysis presented in the text) does lead to systematic overbinding, the differences in relative binding energies are much smaller.

\begin{tabular}{|c|c|c|}
\hline & Default PNO cutoff & Tight PNO cutoff \\
\hline Phe $\cdots$ benzene & -4.5 & -3.5 \\
\hline Phe $\cdots$ pyrazole & -3.6 & -2.8 \\
\hline Phe $\cdots 3$,9-dihydro-purine-2,6-dione & -9.4 & -8.0 \\
\hline Phe $\cdots$ indolizine & -7.6 & -6.1 \\
\hline Tyr*benzene & -4.9 & -4.1 \\
\hline 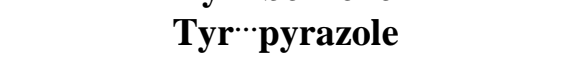 & -4.5 & -3.7 \\
\hline 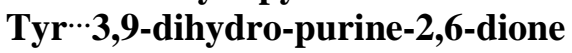 & -11.5 & -10.4 \\
\hline Tyr indolizine & -8.1 & -6.8 \\
\hline Trp $\cdots$ benzene & -6.0 & -4.8 \\
\hline Trp $\cdots$ pyrazole & -5.3 & -4.5 \\
\hline 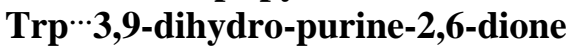 & -14.2 & -12.7 \\
\hline Trp $\cdots$ indolizine & -11.0 & -9.3 \\
\hline
\end{tabular}

Table S5. Absolute energies of 1-58 and Phe, Tyr, and Trp side chains.

\begin{tabular}{ccccc}
\hline & B97D & \multicolumn{2}{c}{ WB97X-D } & DLPNO-CCSD(T) \\
& /def2-TZVPP & \multicolumn{2}{c}{ /def2-TZVPP } & /cc-pVQZ \\
& $\mathbf{E}$ & E (gas) & E (sol) & E \\
\hline $\mathbf{1}$ & -210.096668 & -210.178808 & -210.189604 & -209.852122 \\
$\mathbf{2}$ & -229.952072 & -230.032778 & -230.039509 & -229.699244 \\
$\mathbf{3}$ & -552.975018 & -553.020578 & -553.027779 & -552.322774 \\
$\mathbf{4}$ & -245.967790 & -246.045646 & -246.054237 & -245.706910 \\
$\mathbf{5}$ & -246.001208 & -246.083197 & -246.090690 & -245.744619 \\
$\mathbf{6}$ & -569.016722 & -569.061386 & -569.069597 & -568.357792 \\
$\mathbf{7}$ & -569.019290 & -569.064473 & -569.072071 & -568.361250 \\
$\mathbf{8}$ & -226.129348 & -226.211019 & -226.221475 & -225.878342 \\
\hline
\end{tabular}




\begin{tabular}{|c|c|c|c|c|}
\hline 9 & -226.144658 & -226.228024 & -226.244085 & -225.895822 \\
\hline 10 & -242.179421 & -242.262983 & -242.280949 & -241.925516 \\
\hline 11 & -242.155267 & -242.235126 & -242.247139 & -241.897821 \\
\hline 12 & -242.161498 & -242.242387 & -242.250641 & -241.904530 \\
\hline 13 & -258.189284 & -258.269321 & -258.281161 & -257.926621 \\
\hline 14 & -585.039911 & -585.082622 & -585.090986 & -584.374312 \\
\hline 15 & -232.155881 & -232.248363 & -232.257305 & -231.868309 \\
\hline 16 & -248.191060 & -248.284681 & -248.294485 & -247.898992 \\
\hline 17 & -264.222973 & -264.317279 & -264.326362 & -263.925922 \\
\hline 18 & -264.229148 & -264.324513 & -264.337042 & -263.934249 \\
\hline 19 & -264.195619 & -264.287728 & -264.299436 & -263.896892 \\
\hline 20 & -280.269865 & -280.367792 & -280.384659 & -279.972016 \\
\hline 21 & -280.230019 & -280.323491 & -280.335895 & -279.927210 \\
\hline 22 & -280.206550 & -280.297175 & -280.308735 & -279.901442 \\
\hline 23 & -296.231998 & -296.323955 & -296.336626 & -295.923351 \\
\hline 24 & -296.242932 & -296.335728 & -296.349183 & -295.936179 \\
\hline 25 & -296.213135 & -296.302087 & -296.311640 & -295.901929 \\
\hline 26 & -339.461587 & -339.574456 & -339.595759 & -339.097856 \\
\hline 27 & -363.677947 & -363.824352 & -363.839574 & -363.248480 \\
\hline 28 & -363.665001 & -363.808491 & -363.824608 & -363.233322 \\
\hline 29 & -363.659401 & -363.802413 & -363.816364 & -363.227049 \\
\hline 30 & -379.726569 & -379.874591 & -379.894804 & -379.293152 \\
\hline 31 & -411.800039 & -411.950114 & -411.977100 & -411.356624 \\
\hline 32 & -411.805467 & -411.956257 & -411.978359 & -411.363059 \\
\hline 33 & -420.645877 & -420.796718 & -420.808346 & -420.193577 \\
\hline 34 & -487.045358 & -487.214920 & -487.245387 & -486.537222 \\
\hline 35 & -487.044030 & -487.213515 & -487.242915 & -486.536044 \\
\hline 36 & -562.294002 & -562.486072 & -562.516035 & -561.723336 \\
\hline 37 & -562.279578 & -562.471475 & -562.505255 & -561.709489 \\
\hline 38 & -401.769267 & -401.926265 & -401.940541 & -401.292596 \\
\hline 39 & -417.803970 & -417.962273 & -417.980660 & -417.322430 \\
\hline 40 & -417.807961 & -417.966717 & -417.983648 & -417.327163 \\
\hline 41 & -422.822300 & -422.984430 & -422.996871 & -422.336640 \\
\hline 42 & -476.994797 & -477.171269 & -477.193254 & -476.452180 \\
\hline 43 & -517.262978 & -517.474532 & -517.493431 & -516.648883 \\
\hline 44 & -537.121530 & -537.333086 & -537.349314 & -536.500353 \\
\hline 45 & -860.140754 & -860.316621 & -860.333380 & -859.121839 \\
\hline 46 & -576.413736 & -576.644243 & -576.659581 & -575.745551 \\
\hline 47 & -262.019293 & & -261.755878 & \\
\hline 48 & -262.026969 & & -261.765893 & \\
\hline 49 & -379.720961 & & -379.286460 & \\
\hline 50 & -379.709421 & & -379.272745 & \\
\hline 51 & -379.705139 & & -379.268745 & \\
\hline 52 & -722.602111 & & -721.760232 & \\
\hline
\end{tabular}




\begin{tabular}{|c|c|c|c|c|}
\hline 53 & -385.732484 & & -385.260449 & \\
\hline 54 & -417.803669 & & -417.322329 & \\
\hline 55 & -433.838895 & & -433.353688 & \\
\hline 56 & -477.009374 & & -476.466929 & \\
\hline 57 & -496.846352 & & -496.297408 & \\
\hline 58 & -493.039039 & & -492.491931 & \\
\hline Phe & -271.452382 & -271.568656 & -271.578322 & -271.122443 \\
\hline Trp & -402.970207 & -403.145561 & -403.161022 & -402.503267 \\
\hline Tyr & -346.665273 & -346.802641 & -346.815229 & -346.275561 \\
\hline
\end{tabular}


Table S6. Absolute energies, binding energies, and SAPT0 energy decomposition for all unique dimers of 1-46 with the model toluene (Phe) (n.b. no stacked dimers of $\mathbf{1}$ with Phe were located).

\begin{tabular}{|c|c|c|c|c|c|c|c|c|c|c|}
\hline \multirow[t]{2}{*}{ Het. } & \multirow{2}{*}{$\begin{array}{c}\begin{array}{c}\text { B97D } \\
\text { /def2-TZVPP }\end{array} \\
E \\
\end{array}$} & \multicolumn{2}{|c|}{$\begin{array}{c}\omega B 97 X-D \\
\text { /def2-TZVPP }\end{array}$} & \multicolumn{2}{|c|}{$\begin{array}{c}\text { DLPNO-CCSD(T) } \\
\text { /cc-pVQZ } \\
\end{array}$} & \multicolumn{5}{|c|}{$\begin{array}{c}\text { SAPT0 } \\
\text { /jun-cc-pVDZ } \\
\end{array}$} \\
\hline & & $\mathbf{E}$ (gas) & E (sol) & $\mathbf{E}$ & $\mathbf{E}_{\text {int }}$ & $\mathbf{E}_{\text {total }}$ & $\mathbf{E}_{\text {elec }}$ & $\mathbf{E}_{\text {exch }}$ & $\mathbf{E}_{\text {ind }}$ & $\mathbf{E}_{\text {disp }}$ \\
\hline 2 & -501.416986 & -501.607636 & -501.620867 & -500.828348 & -4.2 & -3.4 & -2.4 & 6.4 & -0.8 & -6.6 \\
\hline 2 & -501.416812 & -501.607472 & -501.620946 & -500.828186 & -4.1 & -3.4 & -2.4 & 6.7 & -0.8 & -6.8 \\
\hline 2 & -501.416424 & -501.607266 & -501.6 & -500.827679 & -3.8 & -2.8 & -1.8 & 6.3 & -0.7 & -6.6 \\
\hline 2 & -501.416744 & -501.607214 & -501.620513 & -500.827621 & -3.7 & -2.8 & -1.7 & 6.1 & -0.8 & -6.3 \\
\hline 2 & -501.416393 & -501.607102 & -501.6 & & -3.6 & -2.6 & -1.9 & 6.3 & -0.7 & -6.2 \\
\hline 2 & -501.415667 & & & & & -2.6 & -1.9 & 5.9 & -0.7 & -6.0 \\
\hline 2 & -501.415236 & -501.605703 & -501.619896 & -500.826325 & -2.9 & -2.0 & -1.3 & 5.1 & -0.6 & -5.3 \\
\hline 3 & -824.4 & -824.595740 & -824. & -823 & -4.5 & -3.9 & -2.5 & 7.1 & -0.9 & -7.6 \\
\hline 3 & -824.439 & -824.5 & -824.6 & -823.4 & -4.5 & -3.8 & -2.7 & 7.4 & -0.9 & -7.7 \\
\hline 3 & & & & & & -3.8 & & 6.8 & -0.8 & -7.4 \\
\hline 3 & -824.43 & -824.595079 & -824.6 & -823.4 & -4.1 & -3.2 & -2.0 & 6.7 & -0.9 & -7.0 \\
\hline 3 & -824.4 & -824.595049 & & & -4.0 & -3.2 & -1.9 & 6.5 & -0.7 & -7.0 \\
\hline 3 & -824.438418 & -824.594094 & -824.608685 & -823.450768 & -3.5 & -2.6 & -1.3 & 5.3 & -0.6 & -6.0 \\
\hline 4 & -517.4 & $-51 \% .02 \angle 003$ & & & & -4.8 & -4.0 & 1.3 & -1.1 & -7.0 \\
\hline 4 & -517.4 & -517.6 & -517. & -516 & -4.1 & -3.7 & -3.1 & 6.6 & -0.9 & -6.3 \\
\hline 4 & -517.4 & -517.620469 & -517.635473 & -516.835631 & -3.9 & -3.7 & -3.0 & 6.5 & -0.9 & -6.2 \\
\hline 5 & -517.4 & -517. & & & -5.1 & -4.3 & -4.2 & 8.4 & -1.1 & -7.5 \\
\hline 5 & -517.466769 & -517.658769 & -517.672878 & -516.874288 & -4.5 & -3.7 & -3.0 & 6.9 & -0.7 & -6.9 \\
\hline 5 & -517.466718 & -517.658602 & -517.672208 & -516.874271 & -4.5 & -3.8 & -3.3 & 7.0 & -0.8 & -6.8 \\
\hline 5 & -517.466372 & -517.658154 & & & -4.2 & -3.5 & -2.8 & 6.1 & -0.7 & -6.0 \\
\hline 5 & -517.465783 & -517.657704 & -517.671926 & -516.873245 & -3.9 & -3.2 & -2.8 & 6.8 & -0.8 & -6.4 \\
\hline 5 & -517.465790 & -517.657735 & -517.671949 & -516.873229 & -3.9 & -3.2 & -2.7 & 6.3 & -0.7 & -6.0 \\
\hline
\end{tabular}




\begin{tabular}{|c|c|c|c|c|c|c|c|c|c|c|}
\hline 5 & -517.465561 & -517.657378 & -517.671773 & -516.872813 & -3.6 & -2.9 & -2.5 & 5.7 & -0.7 & -5.4 \\
\hline 6 & -840.483366 & -840.638003 & -840.652105 & -839.489046 & -5.5 & -5.1 & -4.2 & 8.4 & -1.2 & -8.1 \\
\hline 6 & -840.482514 & -840.637366 & -840.651940 & -839.488404 & -5.1 & -4.7 & -3.5 & 7.7 & -1.0 & -7.9 \\
\hline 6 & -840.482562 & -840.637172 & -840.651473 & -839.488266 & -5.0 & -4.4 & -3.5 & 7.7 & -1.0 & -7.6 \\
\hline 6 & -840.481577 & -840.636310 & -840.651318 & -839.487149 & -4.3 & -4.0 & -2.8 & 6.6 & -0.8 & -7.0 \\
\hline 6 & -840.481495 & -840.636397 & -840.651354 & -839.487110 & -4.3 & -3.9 & -2.9 & 6.8 & -0.9 & -7.0 \\
\hline 7 & -840.485759 & -840.641098 & -840.654757 & -839.492457 & -5.5 & -4.8 & -4.1 & 8.6 & -1.1 & -8.1 \\
\hline 7 & -840.485629 & -840.640704 & -840.654111 & -839.492081 & -5.3 & -4.8 & -4.3 & 8.2 & -1.2 & -7.5 \\
\hline 7 & -840.484666 & -840.639883 & -840.653919 & -839.491303 & -4.8 & -4.1 & -3.2 & 7.6 & -0.9 & -7.7 \\
\hline 7 & -840.484489 & -840.639808 & -840.654127 & -839.491189 & -4.7 & -3.9 & -2.7 & 6.8 & -0.8 & -7.2 \\
\hline 7 & -840.484514 & -840.639707 & -840.654186 & -839.491175 & -4.7 & -4.1 & -3.0 & 6.8 & -0.8 & -7.1 \\
\hline 7 & -840.483847 & -840.639020 & -840.653472 & -839.490345 & -4.2 & -3.5 & -2.5 & 6.5 & -0.8 & -6.7 \\
\hline 7 & -840.483625 & -840.638805 & -840.653393 & -839.490263 & -4.1 & -3.5 & -2.7 & 6.3 & -0.8 & -6.4 \\
\hline 7 & -840.483703 & -840.638958 & -840.653498 & -839.490261 & -4.1 & -3.6 & -2.6 & 6.3 & -0.8 & -6.4 \\
\hline 8 & -497.593404 & -497.784932 & -497.802169 & -497.006507 & -3.6 & -2.7 & -1.7 & 6.2 & -0.7 & -6.5 \\
\hline 10 & -513.644691 & -513.838105 & -513.860673 & -513.054791 & -4.3 & -3.4 & -2.6 & 6.7 & -0.9 & -6.7 \\
\hline 10 & -513.644208 & -513.837584 & -513.860465 & -513.054443 & -4.1 & -3.2 & -2.3 & 6.3 & -0.7 & -6.6 \\
\hline 10 & -513.643108 & -513.836404 & -513.859786 & -513.053151 & -3.3 & -2.4 & -1.5 & 5.9 & -0.6 & -6.1 \\
\hline 12 & -513.627260 & -513.817945 & -513.832180 & -513.034673 & -4.8 & -4.0 & -3.5 & 7.3 & -0.9 & -6.9 \\
\hline 12 & -513.625690 & -513.816384 & -513.831328 & -513.032861 & -3.7 & -3.0 & -2.3 & 5.9 & -0.7 & -5.9 \\
\hline 13 & -529.655538 & -529.845271 & -529.861203 & -529.057421 & -5.2 & -4.5 & -3.8 & 6.9 & -0.9 & -6.7 \\
\hline 13 & -529.654874 & -529.844567 & -529.861645 & -529.056783 & -4.8 & -4.0 & -3.1 & 6.7 & -0.7 & -6.9 \\
\hline 14 & -856.507220 & -856.659818 & -856.673738 & -855.506438 & -6.1 & -5.5 & -4.9 & 8.4 & -1.4 & -7.6 \\
\hline 14 & -856.505269 & -856.658175 & -856.673113 & -855.504854 & -5.1 & -4.5 & -3.5 & 6.5 & -0.8 & -6.6 \\
\hline
\end{tabular}




\begin{tabular}{|c|c|c|c|c|c|c|c|c|c|c|}
\hline 15 & -503.621201 & -503.823858 & -503.839188 & -502.997988 & -4.5 & -3.7 & -2.6 & 7.9 & -0.9 & -8.2 \\
\hline 15 & -503.621249 & -503.823860 & -503.839028 & -502.997898 & -4.5 & -3.7 & -2.5 & 7.8 & -0.9 & -8.1 \\
\hline 15 & -503.619940 & -503.822621 & -503.838567 & -502.996577 & -3.7 & -2.9 & -1.6 & 6.4 & -0.7 & -7.0 \\
\hline 16 & -519.658271 & -519.861920 & -519.877279 & -519.030377 & -5.6 & -5.0 & -4.3 & 9.3 & -1.3 & -8.8 \\
\hline 16 & -519.657383 & -519.861187 & -519.877043 & -519.029642 & -5.2 & -4.4 & -3.3 & 8.3 & -0.9 & -8.5 \\
\hline 16 & -519.657220 & -519.861143 & -519.877182 & -519.029445 & -5.0 & -4.4 & -3.3 & 8.4 & -1.0 & -8.5 \\
\hline 16 & -519.657137 & -519.860866 & -519.876789 & -519.029323 & -4.9 & -4.4 & -3.4 & 8.6 & -1.0 & -8.6 \\
\hline 16 & -519.656143 & -519.859994 & -519.876285 & -519.028223 & -4.3 & -3.6 & -2.7 & 7.5 & -0.9 & -7.5 \\
\hline 16 & -519.656081 & -519.859751 & -519.876018 & -519.028145 & -4.2 & -3.6 & -2.6 & 7.3 & -0.9 & -7.4 \\
\hline 17 & -535.690597 & -535.894821 & -535.909480 & -535.057993 & -6.0 & -5.3 & -5.0 & 10.0 & -1.3 & -9.0 \\
\hline 17 & -535.689788 & -535.893991 & -535.908906 & -535.057021 & -5.4 & -4.7 & -3.9 & 8.3 & -0.9 & -8.1 \\
\hline 17 & -535.688719 & -535.893068 & -535.908494 & -535.055991 & -4.8 & -4.1 & -3.4 & 7.6 & -0.9 & -7.4 \\
\hline 17 & -535.688737 & -535.892954 & -535.908401 & -535.055899 & -4.7 & -4.1 & -3.3 & 7.4 & -0.9 & -7.3 \\
\hline 18 & -535.697233 & -535.902610 & -535.919848 & -535.066471 & -6.1 & -5.6 & -5.3 & 10.3 & -1.4 & -9.2 \\
\hline 18 & -535.695431 & -535.900712 & -535.918221 & -535.064799 & -5.1 & -4.2 & -3.0 & 7.6 & -0.9 & -7.9 \\
\hline 18 & -535.695343 & -535.900679 & -535.918532 & -535.064648 & -5.0 & -4.1 & -2.7 & 7.4 & -0.9 & -7.9 \\
\hline 18 & -535.695364 & -535.900874 & -535.919152 & -535.064427 & -4.9 & -4.5 & -4.1 & 8.6 & -1.0 & -8.0 \\
\hline 19 & -535.664098 & -535.866201 & -535.882772 & -535.029763 & -6.5 & -5.9 & -5.4 & 10.1 & -1.5 & -9.1 \\
\hline 19 & -535.663863 & -535.866090 & -535.883061 & -535.029732 & -6.5 & -5.8 & -5.1 & 10.4 & -1.4 & -9.6 \\
\hline 19 & -535.662053 & -535.864316 & -535.881851 & -535.027581 & -5.2 & -4.6 & -4.1 & 8.6 & -1.1 & -8.0 \\
\hline 20 & -551.737283 & -551.945028 & -551.964925 & -551.103798 & -5.9 & -5.0 & -4.6 & 10.1 & -1.2 & -9.3 \\
\hline 20 & -551.737245 & -551.944916 & -551.964914 & -551.103770 & -5.8 & -5.0 & -4.6 & 9.7 & -1.2 & -8.8 \\
\hline 20 & -551.736449 & -551.944325 & -551.964857 & -551.102950 & -5.3 & -4.5 & -3.8 & 7.6 & -0.8 & -7.5 \\
\hline 21 & -551.699175 & -551.902436 & -551.919221 & -551.060844 & -7.0 & -6.4 & -6.3 & 10.7 & -1.6 & -9.2 \\
\hline 21 & -551.698828 & -551.902077 & -551.919170 & -551.060695 & -6.9 & -6.3 & -5.9 & 10.3 & -1.4 & -9.2 \\
\hline
\end{tabular}




\begin{tabular}{|c|c|c|c|c|c|c|c|c|c|c|}
\hline 21 & -551.698846 & -551.902118 & -551.918996 & -551.060458 & -6.8 & -6.2 & -6.1 & 10.7 & -1.6 & -9.2 \\
\hline 21 & -551.696828 & -551.900066 & -551.917128 & -551.058891 & -5.8 & -4.8 & -4.0 & 8.5 & -1.0 & -8.3 \\
\hline 21 & -551.697146 & -551.900456 & -551.918127 & -551.058613 & -5.6 & -5.1 & -4.8 & 8.8 & -1.3 & -7.8 \\
\hline- & -551.676259 & -551.876658 & -551.892512 & -551.0 & -7.3 & -6.8 & -6.9 & 11.7 & -1.9 & -9.8 \\
\hline 22 & -551.676238 & -551.876624 & -551.892471 & -551.035587 & -7.3 & -6.7 & -6.4 & 11.1 & -1.8 & -9.5 \\
\hline 22 & -551.674302 & -551.874942 & -551.891531 & -551.033608 & -6.1 & -5.6 & -5.4 & 9.5 & -1.4 & -8.3 \\
\hline 23 & -567.701039 & -567.901934 & -567.917901 & -567.056566 & -6.8 & -6.4 & -6.3 & 11.1 & -1.7 & -9.5 \\
\hline 23 & -567.699809 & -567.900778 & -567.917466 & -567.055348 & -6.0 & -5.4 & -4.8 & 10.1 & -1.4 & -9.3 \\
\hline 24 & -567.712467 & -567.914417 & -567.931018 & -567.069990 & -7.1 & -6.5 & -6.4 & 11.8 & -1.9 & -9.9 \\
\hline 24 & -567.712116 & -567.914234 & -567.930492 & -567.069861 & -7.1 & -6.3 & -6.4 & 11.4 & -1.7 & -9.6 \\
\hline 24 & -567.711140 & -567.913343 & -567.929931 & -567.068715 & -6.3 & -5.7 & -5.7 & 9.9 & -1.5 & -8.5 \\
\hline 24 & -567.711174 & -567.913418 & -567.930010 & -567.068598 & -6.3 & -5.7 & -5.7 & 10.2 & -1.5 & -8.8 \\
\hline 25 & -567.683749 & -567.882385 & -567.896186 & -567. & -8.0 & -7.4 & -7.5 & 11.8 & -2.0 & -9.8 \\
\hline 25 & -567.683711 & -567.882200 & -567.896038 & -567.037127 & -8.0 & -7.3 & -7.3 & 11.8 & -2.0 & -9.8 \\
\hline 25 & -567.683907 & -567.882269 & -567.895751 & -567.037077 & -8.0 & -7.5 & -7.6 & 12.0 & -2.2 & -9.7 \\
\hline 25 & -567.682087 & -567.880594 & -567.894620 & -567.035059 & -6.7 & -6.2 & -6.5 & 10.8 & -1.9 & -8.6 \\
\hline 26 & -610.931586 & -611.154854 & -611.178881 & -610 & -7.3 & -6.9 & -6.4 & 10.7 & -1.7 & -9.5 \\
\hline 26 & -610.928977 & -611.152167 & -611.177433 & -610.229898 & -6.0 & -5.3 & -4.0 & 9.5 & -1.5 & -9.2 \\
\hline 26 & -610.928412 & -611.151339 & -611.176725 & -610.229132 & -5.5 & -4.8 & -3.8 & 9.7 & -1.6 & -9.1 \\
\hline 27 & -635.147220 & -635.403994 & -635.423364 & -634.382487 & -7.3 & -6.6 & -4.6 & 11.8 & -1.5 & -12.4 \\
\hline 27 & -635.146461 & -635.403272 & -635.422925 & -634.381652 & -6.7 & -6.2 & -4.6 & 11.9 & -1.4 & -12.0 \\
\hline 27 & -635.146547 & -635.403117 & -635.422693 & -634.381578 & -6.7 & -6.0 & -4.4 & 10.9 & -1.3 & -11.2 \\
\hline 27 & -635.146112 & -635.402921 & -635.422796 & -634.381398 & -6.6 & -5.8 & -3.8 & 10.7 & -1.3 & -11.4 \\
\hline 27 & -635.145605 & -635.402469 & -635.423004 & -634.381092 & -6.4 & -5.4 & -2.8 & 11.4 & -1.4 & -12.6 \\
\hline 27 & -635.145811 & -635.402510 & -635.422715 & -634.380840 & -6.2 & -5.6 & -3.6 & 10.6 & -1.3 & -11.3 \\
\hline
\end{tabular}




\begin{tabular}{|c|c|c|c|c|c|c|c|c|c|c|}
\hline 27 & -635.145884 & -635.402444 & -635.422534 & -634.380727 & -6.2 & -5.6 & -3.9 & 10.7 & -1.4 & -11.0 \\
\hline 27 & -635.144895 & -635.401689 & -635.422071 & -634.380212 & -5.8 & -4.9 & -2.5 & 9.9 & -1.1 & -11.2 \\
\hline 27 & -635.144757 & -635.401601 & -635.422040 & -634.380023 & -5.7 & -4.8 & -2.4 & 9.7 & -1.0 & -11.0 \\
\hline 28 & -635.134696 & -635.388553 & -635.408356 & -634.367158 & -7.1 & -6.9 & -5.5 & 12.1 & -1.7 & -11.8 \\
\hline 28 & -635.133097 & -635.386951 & -635.407793 & -634.366379 & -6.7 & -6.0 & -3.6 & 12.0 & -1.5 & -12.9 \\
\hline 28 & -635.133015 & -635.387082 & -635.408005 & -634.366308 & -6.6 & -6.0 & -3.4 & 11.7 & -1.4 & -12.8 \\
\hline 28 & -635.132776 & -635.386615 & -635.407578 & -634.365816 & -6.3 & -5.7 & -3.5 & 10.5 & -1.4 & -11.3 \\
\hline 28 & -635.132728 & -635.386455 & -635.407075 & -634.365727 & -6.3 & -5.6 & -3.5 & 11.4 & -1.5 & -12.0 \\
\hline 28 & -635.132345 & -635.386081 & -635.407317 & -634.365550 & -6.1 & -5.4 & -3.2 & 11.8 & -1.5 & -12.4 \\
\hline 28 & -635.132427 & -635.386268 & -635.407088 & -634.365327 & -6.0 & -5.5 & -3.5 & 9.9 & -1.3 & -10.7 \\
\hline 28 & -635.131572 & -635.385125 & -635.406130 & -634.364191 & -5.3 & -4.7 & -3.2 & 10.1 & -1.4 & -10.1 \\
\hline 29 & -635.128765 & -635.382867 & -635.400968 & -634.361658 & -7.6 & -7.1 & -5.0 & 11.3 & -1.3 & -12.1 \\
\hline 29 & -635.127945 & -635.381579 & -635.400105 & -634.360757 & -7.1 & -6.3 & -4.4 & 11.7 & -1.4 & -12.3 \\
\hline 29 & -635.128210 & -635.381974 & -635.400234 & -634.360746 & -7.1 & -6.7 & -4.9 & 10.7 & -1.2 & -11.3 \\
\hline 29 & -635.127862 & -635.381688 & -635.400030 & -634.360623 & -7.0 & -6.5 & -4.9 & 10.8 & -1.2 & -11.2 \\
\hline 29 & -635.127798 & -635.381689 & -635.400227 & -634.360554 & -6.9 & -6.2 & -4.5 & 11.6 & -1.3 & -12.0 \\
\hline 29 & -635.127847 & -635.381415 & -635.399775 & -634.360367 & -6.8 & -6.2 & -4.5 & 11.0 & -1.2 & -11.4 \\
\hline 29 & -635.128122 & -635.381597 & -635.400219 & -634.360270 & -6.8 & -6.4 & -4.4 & 10.9 & -1.4 & -11.5 \\
\hline 29 & -635.127410 & -635.381172 & -635.399927 & -634.360224 & -6.7 & -6.1 & -4.4 & 12.7 & -1.5 & -12.9 \\
\hline 29 & -635.127562 & -635.381487 & -635.400122 & -634.360218 & -6.7 & -6.2 & -4.0 & 11.1 & -1.2 & -12.0 \\
\hline 29 & -635.127059 & -635.380882 & -635.399677 & -634.359883 & -6.5 & -6.2 & -4.3 & 9.7 & -1.1 & -10.5 \\
\hline 29 & -635.127133 & -635.380962 & -635.400098 & -634.359837 & -6.5 & -5.7 & -3.2 & 11.0 & -1.2 & -12.3 \\
\hline 30 & -651.194321 & -651.452649 & -651.477336 & -650.425847 & -6.4 & -5.4 & -2.7 & 10.8 & -1.3 & -12.2 \\
\hline 30 & -651.194334 & -651.452762 & -651.477306 & -650.425501 & -6.2 & -5.4 & -3.5 & 10.4 & -1.2 & -11.1 \\
\hline 30 & -651.194240 & -651.452612 & -651.477253 & -650.425390 & -6.1 & -5.4 & -3.9 & 10.3 & -1.4 & -10.5 \\
\hline (1) & -683.272195 & -683.532643 & -683.560668 & -682.493983 & -9.4 & -8.9 & -7.0 & 13.0 & -1.9 & -13.0 \\
\hline 31 & -683.271630 & -683.532055 & -683.560446 & -682.493110 & -8.8 & -8.3 & -7.4 & 13.1 & -1.8 & -12.2 \\
\hline
\end{tabular}




\begin{tabular}{|c|c|c|c|c|c|c|c|c|c|c|}
\hline 31 & -683.271352 & -683.531804 & -683.559851 & -682.492992 & -8.7 & -8.0 & -6.6 & 12.1 & -1.5 & -12.0 \\
\hline 31 & -683.271193 & -683.531693 & -683.559908 & -682.492784 & -8.6 & -7.9 & -6.6 & 12.3 & -1.6 & -12.0 \\
\hline 31 & -683.270901 & -683.531377 & -683.560004 & -682.492668 & -8.5 & -8.1 & -7.0 & 13.2 & -1.7 & -12.6 \\
\hline 2 & -683.275325 & -683.536356 & -683.562708 & -682.498051 & -7.9 & -7.1 & -5.4 & 12.4 & -1.4 & -12.7 \\
\hline 32 & -683.275052 & -683.536188 & -683.562316 & -682.497779 & -7.7 & -7.0 & -5.7 & 12.3 & -1.3 & -12.2 \\
\hline 32 & -683.274338 & -683.535607 & -683.561790 & -682.497120 & -7.3 & -6.4 & -4.6 & 11.2 & -1.1 & -11.9 \\
\hline 32 & -683.274163 & -683.535099 & -683.561653 & -682.496814 & -7.1 & -6.3 & -4.2 & 10.9 & -1.2 & -11.8 \\
\hline 33 & -692.116711 & -692.377552 & -692.393111 & -691.328052 & -7.6 & -6.9 & -7.0 & 12.6 & -1.6 & 10.9 \\
\hline 33 & -692.116695 & -692.377224 & -692.393000 & -691.327457 & -7.2 & -6.4 & -6.1 & 11.4 & -1.6 & -10.1 \\
\hline 33 & -692.116269 & -692.377133 & -692.392895 & -691.327123 & -7.0 & -6.3 & -6.2 & 11.3 & -1.5 & -10.0 \\
\hline 33 & -692.115333 & -692.376033 & -692.392009 & -691.326508 & -6.6 & -6.1 & -6.3 & 11.5 & -1.5 & -9.8 \\
\hline 33 & -692.113966 & -692 & $-692 . ?$ & -691.326230 & -6.4 & -5.4 & -3.6 & 9.7 & -1.0 & -10.5 \\
\hline 33 & -692.113802 & -692.374831 & -692.391703 & -691.325924 & -6.2 & -5.0 & -3.7 & 10.3 & -1.0 & -10.6 \\
\hline 33 & -692.113903 & -692.374933 & -692.391703 & -691.325558 & -6.0 & -5.0 & -3.9 & 10.9 & -1.1 & -10.9 \\
\hline 34 & -758.517274 & -758. & -758.828830 & -757 & -9.4 & -8.5 & -6.9 & 13.3 & -1.5 & -13.4 \\
\hline 34 & -758.516851 & -758.796804 & -758.828590 & -757 & -9.1 & -8.2 & -6.7 & 13.3 & -1.4 & -13.3 \\
\hline 34 & -758.518422 & -758.797221 & -758.827370 & -757.674064 & -9.0 & -9.1 & -9.0 & 14.2 & -2.6 & -11.7 \\
\hline 34 & -758.516612 & -758.796592 & -758.828468 & -757.673820 & -8.9 & -8.0 & -6.5 & 12.7 & -1.4 & -12.8 \\
\hline 34 & -758.516660 & -758.796572 & -758.828470 & -757.6 & -8.8 & -8.0 & -6.7 & 13.4 & -1.4 & -13.2 \\
\hline 34 & -758.517378 & -758.797323 & $-758 . \varepsilon$ & -757.673671 & -8.8 & -8.5 & -7.8 & 13.1 & -1.9 & -12.0 \\
\hline 34 & -758.517334 & -758.796964 & -758.828601 & -757 & -8.7 & -8.6 & -8.0 & 12.7 & -2.0 & -11.3 \\
\hline 34 & -758.517514 & -758.797062 & -758.828025 & -757.673552 & -8.7 & -8.8 & -8.1 & 13.0 & -2.2 & -11.5 \\
\hline 34 & -758.516313 & -758.796218 & -758.827829 & -757.673399 & -8.6 & -7.9 & -6.5 & 12.2 & -1.4 & -12.2 \\
\hline 34 & -758.516371 & -758.796165 & -758.828181 & -757.673297 & -8.6 & -7.8 & -6.4 & 12.9 & -1.4 & -12.9 \\
\hline 35 & -758.516006 & -758.795937 & -758.827979 & -757.673217 & -9.2 & -8.5 & -6.7 & 12.9 & -1.7 & 13.0 \\
\hline 35 & -758.516549 & -758.796172 & -758.827911 & -757.672964 & -9.1 & -9.2 & -8.2 & 12.8 & -2.5 & -11.3 \\
\hline 35 & -758.516201 & -758.796283 & -758.827938 & -757.672963 & -9.1 & -9.0 & -8.1 & 13.5 & -2.1 & -12.3 \\
\hline
\end{tabular}




\begin{tabular}{|c|c|c|c|c|c|c|c|c|c|c|}
\hline 35 & -758.516222 & -758.795833 & -758.827654 & -757.672521 & -8.8 & -8.9 & -8.1 & 12.8 & -2.3 & -11.3 \\
\hline 35 & -758.515203 & -758.795088 & -758.827591 & -757.672311 & -8.7 & -7.8 & -6.5 & 13.1 & -1.7 & -12.8 \\
\hline 35 & -758.514516 & -758.794395 & -758.827498 & -757.671755 & -8.3 & -7.6 & -5.8 & 12.8 & -1.5 & -13.1 \\
\hline 5 & -758.514282 & -758.794133 & -758.827015 & -757.671524 & -8.2 & -7.4 & -5.7 & 12.2 & -1.4 & -12.5 \\
\hline 35 & -758.514210 & -758.794056 & -758.826672 & -757.671446 & -8.1 & -7.3 & -6.0 & 12.5 & -1.4 & -12.5 \\
\hline 35 & -758.514023 & -758.793820 & -758.826632 & -757.671029 & -7.9 & -7.1 & -5.4 & 11.7 & -1.3 & -12.1 \\
\hline 35 & -758.513858 & -758.793711 & -758.826643 & -757.670970 & -7.8 & -7.1 & -5.4 & 12.1 & -1.4 & -12.4 \\
\hline 36 & -833.766656 & -834.069086 & 100266 & -832.8 & -9.4 & -8.7 & -7.6 & 13.9 & -1.4 & -13.6 \\
\hline 36 & -833.766674 & -834.069194 & -834.100126 & -832.860699 & -9.4 & -8.7 & -7.6 & 13.4 & -1.4 & -13.2 \\
\hline 36 & -833.766502 & -834.068951 & -834.099933 & -832.860501 & -9.2 & -8.6 & -7.4 & 13.4 & -1.4 & -13.2 \\
\hline 36 & -833.766448 & -834.068787 & -834.099513 & -832.860202 & -9.1 & -8.5 & -7.6 & 13.9 & -1.5 & -13.3 \\
\hline 36 & -833.766110 & -834.0 & -834.0 & -832.8 & -9.0 & -8.6 & -7.5 & 13.2 & -1.4 & -12.8 \\
\hline 36 & -833.766024 & -834.068344 & -834.098911 & -832.860030 & -8.9 & -8.5 & -7.5 & 12.9 & -1.5 & -12.4 \\
\hline 36 & -833.765603 & -834.068051 & -834.099135 & -832.859947 & -8.9 & -7.8 & -6.4 & 12.2 & -1.2 & -12.5 \\
\hline 36 & -833.765739 & -834.068372 & -834.099285 & -832.859856 & -8.8 & -8.2 & -7.1 & 12.8 & -1.2 & -12.7 \\
\hline 36 & -833.765699 & -834.068058 & -834.099467 & -832.859731 & -8.8 & -7.7 & -6.9 & 13.0 & -1.4 & -12.3 \\
\hline 36 & -833.765290 & -834.067953 & -834.099301 & -832.859520 & -8.6 & -7.4 & -6.7 & 13.2 & -1.4 & -12.4 \\
\hline 36 & -833.764976 & -834.067357 & -834.098188 & -832.858886 & -8.2 & -7.5 & -6.7 & 11.7 & -1.3 & -11.1 \\
\hline 37 & -833 & -834.053534 & 990321 & -83 & -0.0 & -7.9 & -7.0 & 13.5 & -1.7 & -12.7 \\
\hline 37 & -833.751121 & -834.053002 & -834.089854 & -832.8 & -8.5 & -7.6 & -6.5 & 12.9 & -1.8 & -12.3 \\
\hline 50 & -673. & -673 & -673 & -672 & -8.1 & -7.7 & -5.1 & 12.7 & -1.5 & -15.1 \\
\hline 38 & -673.238265 & -673.506030 & -673.524897 & -672.426870 & -7.4 & -6.9 & -4.4 & 11.4 & -1.2 & -12.7 \\
\hline 38 & -673.238108 & -673.505710 & -673.524635 & -672.426650 & -7.3 & -6.8 & -4.5 & 11.8 & -1.2 & -12.8 \\
\hline 38 & -673.238109 & -673.505976 & -673.524857 & -672.426592 & -7.2 & -6.7 & -4.5 & 11.9 & -1.2 & -12.8 \\
\hline 38 & -673.237975 & -673.505778 & -673.524783 & -672.426499 & -7.2 & -6.6 & -4.0 & 11.0 & -1.1 & -12.4 \\
\hline 38 & -673.237697 & -673.505374 & -673.524581 & -672.426474 & -7.2 & -6.3 & -3.4 & 11.5 & -1.2 & -13.2 \\
\hline 38 & -673.237978 & -673.505674 & -673.524340 & -672.426437 & -7.2 & -6.7 & -4.8 & 12.2 & -1.3 & -12.8 \\
\hline 38 & -673.237840 & -673.505618 & -673.524502 & -672.426286 & -7.1 & -6.7 & -4.5 & 12.1 & -1.3 & -13.0 \\
\hline
\end{tabular}




\begin{tabular}{|c|c|c|c|c|c|c|c|c|c|c|}
\hline 38 & -673.237835 & -673.505626 & -673.524576 & -672.425908 & -6.8 & -6.2 & -4.0 & 11.1 & -1.3 & -11.9 \\
\hline 38 & -673.237867 & -673.505417 & -673.524137 & -672.425802 & -6.8 & -6.3 & -4.9 & 11.4 & -1.4 & -11.4 \\
\hline 38 & -673.237209 & -673.504844 & -673.523982 & -672.425676 & -6.7 & -6.1 & -3.5 & 10.1 & -1.0 & -11.7 \\
\hline 38 & -673.237600 & -673.505101 & -673.524191 & -672.425409 & -6.5 & -5.8 & -3.7 & 10.1 & -1.2 & -11.1 \\
\hline 39 & -689.275273 & -689.544114 & -689.565476 & -688.459085 & -8.9 & -8.6 & -5.9 & 12.6 & -1.7 & -13.6 \\
\hline 39 & -689.274737 & -689.543749 & -689.565366 & -688.458779 & -8.7 & -8.3 & -5.8 & 12.8 & -1.6 & -13.7 \\
\hline 39 & -689.273964 & -689.542853 & -689.564801 & -688.457651 & -8.0 & -7.6 & -5.5 & 12.4 & -1.4 & -13.1 \\
\hline 39 & -689.273640 & -689.542482 & -689.564575 & -688.457353 & -7.8 & -7.4 & -5.3 & 12.1 & -1.3 & -12.9 \\
\hline 39 & -689.273440 & -689.542239 & -689.564436 & -688 & -7.8 & -7.4 & -5.4 & 12.4 & -1.4 & -13.0 \\
\hline 39 & -689.274021 & -689.543111 & -689.564860 & -688.457220 & -7.7 & -7.3 & -5.4 & 12.0 & -1.4 & -12.4 \\
\hline 39 & -689.272333 & -689.541210 & -689.563903 & -688.456498 & -7.3 & -6.3 & -3.4 & 11.3 & -1.2 & -13.0 \\
\hline 39 & -689.272510 & -689.541117 & -689.563334 & -688.456121 & -7.1 & -6.8 & -4.8 & 11.4 & -1.2 & -12.1 \\
\hline 39 & -689.272873 & -689.541540 & -689.563787 & -688.455789 & -6.9 & -6.5 & -5.1 & 11.5 & -1.4 & -11.5 \\
\hline 40 & -68 & 48401 & -689 & -688 & -8.6 & -8.5 & -6.6 & 14.2 & -1.6 & 14.5 \\
\hline 40 & -689.278297 & -689.547648 & -689.567667 & -688.462648 & -8.2 & -7.9 & -6.0 & 12.8 & -1.4 & -13.3 \\
\hline 40 & -689.278535 & -689.547872 & -689.568115 & -688.462640 & -8.2 & -7.9 & -6.7 & 13.9 & -1.7 & -13.4 \\
\hline 40 & -689.277652 & -689.547182 & -689.567953 & -688.462202 & -7.9 & -7.6 & -5.9 & 13.3 & -1.4 & -13.5 \\
\hline 40 & -689.277936 & -689.547519 & -689.568199 & -688 & -7.9 & -7.6 & -5.9 & 13.2 & -1.5 & -13.5 \\
\hline 40 & -689 . & 47244 & -689.568114 & -688 & -7.8 & -7.4 & -5.3 & 11.9 & -1.3 & -12.8 \\
\hline 40 & -689.277211 & -689.546602 & -689.567623 & -688 & -7.7 & -6.8 & -4.2 & 11.7 & -1.2 & -13.1 \\
\hline 40 & -689.276867 & -689.545920 & -689.566814 & -688.461212 & -7.3 & -7.0 & -5.1 & 12.3 & -1.3 & -12.9 \\
\hline 40 & -689.276925 & -689.545852 & -689.567003 & -688.460567 & -6.9 & -6.4 & -5.2 & 11.7 & -1.5 & -11.5 \\
\hline 41 & -694.293276 & -694.565692 & -694.583109 & -693.471313 & -7.7 & -7.4 & -6.8 & 13.7 & -1.8 & -12.5 \\
\hline 41 & -694.292916 & -694.565140 & -694.582599 & -693.470930 & -7.4 & -7.1 & -6.7 & 13.3 & -1.7 & -12.0 \\
\hline 41 & -694.293017 & -694.565280 & -694.582839 & -693.470643 & -7.3 & -6.8 & -5.9 & 11.8 & -1.6 & -11.1 \\
\hline 41 & -694.291221 & -694.563994 & -694.582205 & -693.470609 & -7.2 & -6.5 & -4.2 & 11.2 & -1.3 & -12.2 \\
\hline 41 & -694.291978 & -694.564633 & -694.582406 & -693.470352 & -7.1 & -6.6 & -5.5 & 12.9 & -1.6 & -12.5 \\
\hline 41 & -694.291266 & -694.563818 & -694.581937 & -693.470291 & -7.0 & -6.4 & -4.6 & 11.4 & -1.4 & -11.9 \\
\hline
\end{tabular}




\begin{tabular}{|c|c|c|c|c|c|c|c|c|c|c|}
\hline 41 & -694.291970 & -694.564272 & -694.581972 & -693.470115 & -6.9 & -6.7 & -6.2 & 12.6 & -1.6 & -11.4 \\
\hline 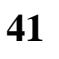 & -694.291383 & -694.564004 & -694.581768 & -693.470088 & -6.9 & -6.4 & -4.9 & 11.4 & -1.3 & -11.6 \\
\hline 1 & -694.291245 & -694.563920 & -694.581904 & -693.470004 & -6.9 & -6.3 & -4.2 & 10.6 & -1.3 & -11.4 \\
\hline 41 & -694.291058 & -694.563427 & -694.581245 & -693.469613 & -6.6 & -6.3 & -5.0 & 11.3 & -1.4 & -11.3 \\
\hline 41 & -694.291388 & -694.563893 & -694.581684 & -693.469601 & -6.6 & -6.2 & -5.2 & 12.1 & -1.5 & -11.6 \\
\hline 41 & -694.290416 & -694.562863 & -694.581279 & -693. & -6.6 & -5.7 & -3.2 & 10.4 & -1.1 & -11.7 \\
\hline 41 & -694.290655 & -694.563343 & -694 & -693 & -6.5 & -5.7 & -3.8 & 10.6 & -1.2 & -11.3 \\
\hline 41 & -694.290314 & -694.562812 & -694 & -693 & -6.5 & -5.8 & -3.9 & 11.0 & -1.2 & -11.7 \\
\hline 41 & -694.290366 & -694.562898 & -694.581316 & 9308 & -6.4 & -5.7 & -3.9 & 10.8 & -1.2 & -11.4 \\
\hline 41 & -694.290234 & -694.562870 & -694.581278 & -693.469266 & -6.4 & -5.6 & -3.6 & 10.3 & -1.0 & -11.3 \\
\hline 41 & -694.2 & -694.5 & -694.5 & -693 & -6.3 & -5.5 & -3.5 & 10.6 & -1.1 & -11.4 \\
\hline 41 & -694.290456 & -694.562870 & -694.581023 & -693 & -6.3 & -5.6 & -4.2 & 11.5 & -1.3 & -11.6 \\
\hline 41 & -694.290058 & -694.562138 & -694.5 & -693 & -6.3 & -5.6 & -3.1 & 9.2 & -1.0 & -10.7 \\
\hline 41 & -694 & -694 & -69 & -69 & -6.1 & -5.1 & -2.6 & 9.8 & -0.9 & 1.4 \\
\hline 41 & -694.289854 & -694.562311 & -694.580783 & -693.4 & -6.1 & -5.2 & -3.1 & 9.8 & -1.0 & -10.9 \\
\hline 41 & -694.291343 & -694.5 & -694. & -693 & -6.0 & -6.0 & -4.2 & 11.0 & -1.4 & -11.4 \\
\hline 41 & -694.289854 & -694 & -694 & -693 & -5.9 & -4.9 & -2.5 & 10.1 & -1.1 & -11.5 \\
\hline 41 & -694.2 & -694 & -694 & -693 & -5.7 & -5.5 & -4.2 & 10.4 & -1.2 & -10.4 \\
\hline 42 & -748.466984 & -748.7 & -748. & -747 & -9.1 & -8.6 & -6.8 & 14.4 & -1.8 & -14.4 \\
\hline 42 & -748.466240 & -748.753113 & -748.778432 & -747.588325 & -8.6 & -8.1 & -5.9 & 12.7 & -1.6 & -13.2 \\
\hline 42 & -748.464693 & -748.7 & -748.7 & -747.5 & -8.0 & -7.2 & -4.7 & 12.9 & -1.5 & -14.0 \\
\hline 42 & -748.465342 & -748 & -748 & -747 & -7.9 & -7.4 & -5.8 & 12.5 & -1.4 & -12.7 \\
\hline 42 & -748.465117 & -748. & -748 & -747 & -7.9 & -7.2 & -5.5 & 12.9 & -1.5 & -13.1 \\
\hline 42 & -748 & -748. & 77788 & -747 & -7.8 & -7.0 & -4.6 & 13.0 & -1.5 & -13.8 \\
\hline 42 & -748.464717 & -748.751791 & -748.777469 & -747.586488 & -7.4 & -7.1 & -5.9 & 13.2 & -1.8 & -12.6 \\
\hline 43 & -788.737083 & -789.058351 & -789. & -787.787250 & -10.0 & -10.0 & -8.9 & 16.2 & -2.5 & -14.9 \\
\hline 43 & -788.736932 & -789.058167 & -789.078701 & -787.787147 & -9.9 & -10.0 & -8.8 & 16.3 & -2.5 & -15.0 \\
\hline 43 & -788.736683 & -789.057946 & -789.078510 & -787.786684 & -9.6 & -9.7 & -8.6 & 14.7 & -2.2 & -13.7 \\
\hline 43 & -788.733610 & -789.055802 & -789.078561 & -787.784916 & -8.5 & -7.9 & -4.7 & 14.0 & -1.4 & -15.9 \\
\hline
\end{tabular}




\begin{tabular}{|c|c|c|c|c|c|c|c|c|c|c|}
\hline 43 & -788.733601 & -789.055648 & -789.078468 & -787.784915 & -8.5 & -7.9 & -4.7 & 14.1 & -1.4 & -15.8 \\
\hline 43 & -788.733464 & -789.055709 & -789.078247 & -787.784624 & -8.3 & -7.7 & -4.8 & 13.4 & -1.4 & -14.9 \\
\hline 43 & -788.733764 & -789.055431 & -789.077300 & -787.784442 & -8.2 & -7.9 & -6.0 & 13.6 & -1.6 & -13.9 \\
\hline 44 & -808.592210 & -808.914326 & -808.934367 & -807.636742 & -8.8 & -8.2 & -5.1 & 13.0 & -1.2 & -14.9 \\
\hline 44 & -808.592317 & -808.914500 & -808.934564 & -807.636468 & -8.6 & -7.9 & -5.1 & 13.4 & -1.3 & -14.8 \\
\hline 44 & -808.592022 & -808.914147 & -808.934125 & -807.636193 & -8.4 & -7.7 & -5.0 & 13.1 & -1.3 & -14.4 \\
\hline 44 & -808.592092 & -808.914412 & -808.934491 & -807.635993 & -8.3 & -7.7 & -5.1 & 13.1 & -1.4 & -14.4 \\
\hline 44 & -808.591855 & -808.914197 & -808.934454 & -807.635942 & -8.2 & -7.4 & -4.4 & 12.3 & -1.2 & -14.2 \\
\hline 44 & -808.591552 & -808.913682 & -808.933911 & -807.635893 & -8.2 & -7.6 & -4.8 & 12.4 & -1.1 & -14.1 \\
\hline 44 & -808.591502 & -808.913796 & -808.934019 & -807.635869 & -8.2 & -7.7 & -4.9 & 12.8 & -1.1 & -14.4 \\
\hline 44 & -808.591556 & -808.913782 & -808.933955 & -807.635753 & -8.1 & -7.6 & -5.0 & 13.1 & -1.2 & -14.5 \\
\hline 44 & -808.591426 & -808.913503 & -808.933690 & -807.635699 & -8.1 & -7.6 & -4.8 & 12.7 & -1.1 & -14.5 \\
\hline 45 & -1131.611610 & -1131.898331 & -1131.918956 & -1130.258872 & -9.2 & -8.8 & -5.3 & 13.6 & -1.3 & -15.8 \\
\hline 45 & -1131.611923 & -1131.898882 & -1131.919489 & -1130.258558 & -9.0 & -8.5 & -5.4 & 13.6 & -1.4 & -15.2 \\
\hline 45 & -1131.611677 & -1131.898247 & -1131.918924 & -1130.258195 & -8.7 & -8.3 & -5.3 & 13.2 & -1.4 & -14.9 \\
\hline 45 & -1131.611201 & -1131.897611 & -1131.918385 & -1130.258042 & -8.6 & -8.0 & -4.5 & 13.1 & -1.4 & -15.2 \\
\hline 45 & -1131.610951 & -1131.897613 & -1131.918482 & -1130.257928 & -8.6 & -8.1 & -4.8 & 12.5 & -1.2 & -14.7 \\
\hline 45 & -1131.610959 & -1131.897497 & -1131.918268 & -1130.257898 & -8.5 & -8.1 & -5.0 & 13.3 & -1.3 & -15.1 \\
\hline 45 & -1131.610903 & -1131.897667 & -1131.918562 & -1130.257758 & -8.5 & -8.0 & -4.9 & 13.0 & -1.2 & -14.9 \\
\hline 45 & -1131.610594 & -1131.897199 & -1131.918307 & -1130.257399 & -8.2 & -7.8 & -4.3 & 12.3 & -1.1 & -14.8 \\
\hline 46 & -847.887500 & -848.228257 & -848.248141 & -846.883657 & -9.8 & -9.5 & -7.8 & 15.3 & -1.9 & -15.2 \\
\hline 46 & -847.886835 & -848.227689 & -848.247749 & -846.882832 & -9.3 & -9.0 & -7.0 & 14.4 & -1.7 & -14.8 \\
\hline 46 & -847.885682 & -848.226756 & -848.247254 & -846.882760 & -9.3 & -8.4 & -6.6 & 15.6 & -1.5 & -15.9 \\
\hline 46 & -847.885675 & -848.227097 & -848.247806 & -846.882682 & -9.2 & -8.0 & -5.8 & 15.6 & -1.5 & -16.3 \\
\hline 46 & -847.885941 & -848.226899 & -848.247449 & -846.882487 & -9.1 & -8.4 & -6.7 & 16.4 & -1.7 & -16.4 \\
\hline 46 & -847.886208 & -848.226892 & -848.247058 & -846.882326 & -9.0 & -8.6 & -7.1 & 14.2 & -1.7 & -14.0 \\
\hline 46 & -847.885753 & -848.226543 & -848.246972 & -846.882147 & -8.9 & -8.2 & -6.4 & 14.6 & -1.5 & -14.9 \\
\hline 46 & -847.884874 & -848.225420 & -848.246106 & -846.881113 & -8.2 & -8.1 & -6.6 & 13.3 & -1.5 & -13.3 \\
\hline
\end{tabular}


Table S7. Absolute energies, binding energies, and SAPT0 energy decomposition for all unique dimers of 1-46 with the model pmethylphenol (Tyr). (n.b. no stacked dimers of 1 with Tyr were located).

\begin{tabular}{|c|c|c|c|c|c|c|c|c|c|c|}
\hline \multirow{2}{*}{ Het. } & \multirow{2}{*}{$\begin{array}{c}\begin{array}{c}\text { B97D } \\
\text { /def2-TZVPP }\end{array} \\
E\end{array}$} & \multicolumn{2}{|c|}{$\begin{array}{c}\omega B 97 X-D \\
/ \text { def2-TZVPP }\end{array}$} & \multicolumn{2}{|c|}{$\begin{array}{c}\text { DLPNO-CCSD(T) } \\
\text { /cc-pVQZ }\end{array}$} & \multicolumn{5}{|c|}{$\begin{array}{c}\text { SAPT0 } \\
\text { /jun-cc-pVDZ }\end{array}$} \\
\hline & & E (gas) & E (sol) & $\mathbf{E}$ & $\mathbf{E}_{\text {int }}$ & $\mathbf{E}_{\text {total }}$ & $\mathbf{E}_{\text {elec }}$ & $\mathbf{E}_{\text {exch }}$ & $\mathbf{E}_{\text {ind }}$ & $\mathbf{E}_{\text {disp }}$ \\
\hline 2 & -576.632020 & -576.841988 & -576.857578 & -575.981715 & -4.3 & -3.7 & -2.9 & 6.9 & -0.8 & -6.9 \\
\hline 2 & -576.631956 & -576.842033 & -576.857885 & -575.981453 & -4.2 & -3.5 & -2.9 & 7.7 & -0.9 & -7.4 \\
\hline 2 & -576.631409 & -576.841169 & -576.857334 & -575.980938 & -3.8 & -3.0 & -2.1 & 6.3 & -0.7 & -6.4 \\
\hline 2 & -576.631611 & -576.841425 & -576.857182 & -575.980774 & -3.7 & -3.0 & -2.0 & 6.4 & -0.8 & -6.6 \\
\hline 3 & -899.655715 & -899.830894 & -899.846973 & -898.606667 & -5.2 & -4.9 & -4.0 & 8.3 & -1.1 & -8.1 \\
\hline 3 & -899.655523 & -899.830677 & -899.846914 & -898.606327 & -5.0 & -4.5 & -3.6 & 7.9 & -1.0 & -7.8 \\
\hline 3 & -899.655225 & -899.830225 & -899.846588 & -898.606059 & -4.8 & -4.3 & -3.2 & 8.1 & -1.0 & -8.2 \\
\hline 3 & -899.655036 & -899.830133 & -899.846516 & -898.605708 & -4.6 & -4.0 & -2.9 & 7.7 & -0.9 & -7.9 \\
\hline 3 & -899.654407 & -899.829395 & -899.846107 & -898.605207 & -4.3 & -3.6 & -2.2 & 6.9 & -0.8 & -7.5 \\
\hline 3 & -899.654469 & -899.829351 & -899.845619 & -898.605050 & -4.2 & -3.5 & -2.2 & 7.1 & -0.9 & -7.5 \\
\hline 4 & -592.649010 & -592.856153 & -592.873033 & -591.990622 & -5.1 & -4.8 & -4.1 & 7.8 & -1.1 & -7.4 \\
\hline 4 & -592.648967 & -592.856042 & -592.872966 & -591.990577 & -5.1 & -4.9 & -4.3 & 7.8 & -1.1 & -7.3 \\
\hline 4 & -592.648823 & -592.855968 & -592.873032 & -591.990280 & -4.9 & -4.7 & -4.1 & 7.8 & -1.0 & -7.4 \\
\hline 5 & -592.682573 & -592.893844 & -592.909808 & -592.028354 & -5.1 & -4.5 & -4.5 & 9.1 & -1.1 & -7.9 \\
\hline 5 & -592.681785 & -592.893012 & -592.909491 & -592.027808 & -4.8 & -4.0 & -3.3 & 7.0 & -0.8 & -7.0 \\
\hline 5 & -592.681398 & -592.892768 & -592.909293 & -592.027551 & -4.6 & -4.1 & -3.2 & 6.3 & -0.6 & -6.6 \\
\hline 5 & -592.681766 & -592.892873 & -592.909468 & -592.027506 & -4.6 & -3.9 & -3.6 & 6.4 & -0.7 & -6.0 \\
\hline 5 & -592.681464 & -592.892382 & -592.908946 & -592.027191 & -4.4 & -3.7 & -3.3 & 7.0 & -0.8 & -6.6 \\
\hline
\end{tabular}




\begin{tabular}{|c|c|c|c|c|c|c|c|c|c|c|}
\hline 6 & -915.698442 & -915.872585 & -915.889260 & -914.642577 & -5.8 & -5.7 & -5.0 & 9.2 & -1.2 & -8.7 \\
\hline 6 & -915.698216 & -915.872208 & -915.888848 & -914.642041 & -5.5 & -5.1 & -4.3 & 8.7 & -1.2 & -8.3 \\
\hline 6 & -915.697629 & -915.871591 & -915.888637 & -914.641752 & -5.3 & -4.9 & -4.0 & 8.6 & -1.1 & -8.4 \\
\hline 6 & -915.697509 & -915.871507 & -915.888352 & -914.641624 & -5.2 & -4.7 & -3.8 & 8.0 & -1.1 & -7.8 \\
\hline 7 & -915.700746 & -915.875293 & -915.891418 & -914.645649 & -5.5 & -5.1 & -4.4 & 8.9 & -1.2 & -8.5 \\
\hline 7 & -915.700501 & -915.874865 & -915.891120 & -914.645592 & -5.5 & -4.9 & -4.3 & 8.7 & -1.2 & -8.1 \\
\hline 7 & -915.699578 & -915.873957 & -915.890435 & -914.644468 & -4.8 & -4.2 & -3.5 & 8.3 & -1.0 & -8.0 \\
\hline 7 & -915.699147 & -915.873724 & -915.890659 & -914.644088 & -4.6 & -3.9 & -2.7 & 7.0 & -0.8 & -7.4 \\
\hline 8 & -572.809556 & -573.020579 & -573.039996 & -572.161142 & -4.5 & -3.8 & -2.9 & 7.4 & -1.0 & -7.3 \\
\hline 10 & -588.859520 & -589.072255 & -589.097143 & -588.207959 & -4.3 & -3.6 & -2.8 & 6.9 & -0.8 & -6.8 \\
\hline 12 & -588.842575 & -589.052535 & -589.069237 & -588.188293 & -5.1 & -4.6 & -4.1 & 7.9 & -0.9 & -7.4 \\
\hline 12 & -588.842029 & -589.052072 & -589.068926 & -588.187714 & -4.8 & -4.0 & -3.6 & 7.8 & -0.9 & -7.2 \\
\hline 13 & -604.869924 & -605.078872 & -605.097777 & -604.209853 & -4.8 & -4.1 & -3.3 & 6.8 & -0.8 & -6.8 \\
\hline 14 & -931.722086 & -931.894006 & -931.910789 & -930.659935 & -6.3 & -5.8 & -5.2 & 8.9 & -1.3 & -8.2 \\
\hline 15 & -578.836664 & -579.058693 & -579.076281 & -578.151716 & -4.9 & -4.4 & -3.6 & 8.6 & -1.1 & -8.3 \\
\hline 15 & -578.836332 & -579.058270 & -579.076012 & -578.151199 & -4.6 & -3.9 & -2.9 & 8.6 & -1.0 & -8.6 \\
\hline 16 & -594.873268 & -595.096300 & -595.114251 & -594.183779 & -5.8 & -5.2 & -4.7 & 10.2 & -1.3 & -9.4 \\
\hline 16 & -594.872914 & -595.096068 & -595.114228 & -594.183338 & -5.5 & -5.0 & -4.6 & 10.3 & -1.2 & -9.5 \\
\hline 16 & -594.872791 & -595.095868 & -595.114023 & -594.183276 & -5.5 & -5.1 & -4.4 & 9.4 & -1.1 & -9.0 \\
\hline 16 & -594.872521 & -595.095523 & -595.113770 & -594.182836 & -5.2 & -4.6 & -3.8 & 9.3 & -1.1 & -8.9 \\
\hline 16 & -594.872215 & -595.095119 & -595.113544 & -594.182754 & -5.1 & -4.5 & -3.8 & 9.6 & -1.1 & -9.2 \\
\hline 17 & -610.906213 & -611.129662 & -611.146627 & -610.211745 & -6.4 & -5.9 & -5.9 & 11.1 & -1.4 & -9.7 \\
\hline
\end{tabular}




\begin{tabular}{|c|c|c|c|c|c|c|c|c|c|c|}
\hline 17 & -610.905398 & -611.128973 & -611.145980 & -610.211171 & -6.1 & -5.5 & -5.3 & 9.8 & -1.1 & -9.0 \\
\hline 17 & -610.905306 & -611.128781 & -611.146151 & -610.210804 & -5.8 & -5.1 & -4.8 & 10.1 & -1.3 & -9.1 \\
\hline 18 & -610.912575 & -611.137072 & -611.156969 & -610.219991 & -6.4 & -6.0 & -5.9 & 11.2 & -1.4 & -9.9 \\
\hline 18 & -610.912080 & -611.136655 & -611.156474 & -610.219546 & -6.1 & -5.6 & -5.3 & 10.4 & -1.3 & -9.4 \\
\hline 18 & -610.912212 & -611.136667 & -611.155801 & -610.219535 & -6.1 & -6.1 & -6.8 & 11.0 & -1.4 & -8.9 \\
\hline 18 & -610.911075 & -611.135553 & -611.155275 & -610.218892 & -5.7 & -5.0 & -4.2 & 9.3 & -1.1 & -9.0 \\
\hline 18 & -610.910272 & -611.134962 & -611.154886 & -610.218096 & -5.2 & -4.4 & -3.3 & 7.8 & -0.8 & -8.0 \\
\hline 18 & -610.910005 & -611.134491 & -611.154720 & -610.217594 & -4.9 & -4.0 & -3.1 & 8.7 & -1.1 & -8.5 \\
\hline 19 & -610.879175 & -611.100455 & -611.119576 & -610.183066 & -6.7 & -6.1 & -6.2 & 11.8 & -1.6 & -10.2 \\
\hline 19 & -610.878850 & -611.100087 & -611.119329 & -610.182887 & -6.5 & -6.0 & -5.3 & 10.2 & -1.5 & -9.4 \\
\hline 19 & -610.878278 & -611.099646 & -611.119253 & -610.182136 & -6.1 & -5.6 & -5.2 & 10.7 & -1.4 & -9.6 \\
\hline 20 & -626.953247 & -627.180050 & -627.201699 & -626.258120 & -6.6 & -6.0 & -6.4 & 11.1 & -1.4 & -9.4 \\
\hline 20 & -626.952953 & -627.179859 & -627.202286 & -626.257606 & -6.3 & -5.5 & -5.4 & 10.6 & -1.4 & -9.4 \\
\hline 20 & -626.952344 & -627.179143 & -627.201769 & -626.256911 & -5.9 & -5.0 & -4.8 & 10.7 & -1.4 & -9.5 \\
\hline 21 & -626.915043 & -627 & -627 & -62 & -7.6 & -7.2 & -7.5 & 11.8 & -1.6 & -9.9 \\
\hline 21 & -626.914161 & -627.136438 & -627.155943 & -626.213986 & -7.0 & -6.5 & -6.5 & 11.6 & -1.8 & -10.0 \\
\hline 21 & -626.913636 & -627.136148 & -627.155342 & -626.213560 & -6.8 & -6.2 & -6.2 & 10.8 & -1.4 & -9.4 \\
\hline 21 & -626.912722 & -627.135018 & -627.154236 & -626.212751 & -6.3 & -5.4 & -5.1 & 10.2 & -1.4 & -9.3 \\
\hline 22 & -626.892786 & -627.1 & -627.129940 & -626.190714 & -8.6 & -8.4 & -9.0 & 11.7 & -1.9 & -9.2 \\
\hline 22 & -626.891774 & -627.111400 & -627.129912 & -626.189406 & -7.8 & -7.3 & -7.6 & 12.7 & -1.9 & -10.5 \\
\hline 22 & -626.891332 & -627.110725 & -627.129213 & -626.188784 & -7.4 & -6.8 & -6.8 & 12.3 & -2.0 & -10.3 \\
\hline 22 & -626.891401 & -627.110987 & -627.129443 & -626.188781 & -7.4 & -6.9 & -7.3 & 12.8 & -2.0 & -10.4 \\
\hline 23 & -642.916648 & -643.136620 & -643.154356 & -642.210452 & -7.2 & -6.7 & -7.4 & 12.0 & -1.6 & -9.7 \\
\hline 23 & -642.916933 & -643.136689 & -643.154861 & -642.210410 & -7.2 & -6.7 & -7.3 & 12.7 & -1.8 & -10.3 \\
\hline 23 & -642.915779 & -643.135388 & -643.154264 & -642.209181 & -6.4 & -5.9 & -6.0 & 11.8 & -1.8 & -9.9 \\
\hline
\end{tabular}




\begin{tabular}{|c|c|c|c|c|c|c|c|c|c|c|}
\hline 23 & -642.915580 & -643.135105 & -643.153448 & -642.208994 & -6.3 & -6.1 & -6.1 & 11.5 & -1.7 & -9.8 \\
\hline 24 & -642.928198 & -643.149628 & -643.167722 & -642.224161 & -7.8 & -7.3 & -7.8 & 12.1 & -1.8 & -9.8 \\
\hline 24 & -642.927929 & -643.149213 & -643.167788 & -642.223567 & -7.4 & -6.8 & -7.2 & 12.3 & -1.9 & -10.0 \\
\hline 24 & -642.927477 & -643.148163 & -643.166959 & -642.222813 & -6.9 & -6.3 & -6.8 & 12.5 & -2.0 & -10.0 \\
\hline 24 & -642.926711 & -643.147966 & -643.167512 & -642.222726 & -6.9 & -6.1 & -5.7 & 10.5 & -1.7 & -9.3 \\
\hline 25 & -642.900107 & -643.117444 & -643.132935 & -642.191376 & -8.7 & -8.4 & -9.0 & 13.3 & -2.3 & -10.4 \\
\hline 25 & -642.899095 & -643.116363 & -643.132479 & -642.190259 & -8.0 & -7.6 & -8.0 & 13.2 & -2.3 & -10.4 \\
\hline 25 & -642.897825 & -643.115251 & -643.131662 & -642.188857 & -7.1 & -6.3 & -6.5 & 11.5 & -1.9 & -9.4 \\
\hline 25 & -642.897623 & -643.114925 & -643.131645 & -642.188482 & -6.9 & -6.2 & -6.6 & 12.2 & -2.1 & -9.7 \\
\hline 26 & -686.145623 & -686.387786 & -686.413899 & -685.385005 & -7.3 & -6.9 & -6.1 & 11.7 & -2.3 & -10.2 \\
\hline 26 & -686.146432 & -686.388252 & -686.414309 & -685.384949 & -7.2 & -8.0 & -8.0 & 13.0 & -2.5 & -10.4 \\
\hline 26 & -686.146146 & -686.388456 & -686.415600 & -685.384636 & -7.0 & -6.9 & -6.6 & 11.5 & -1.8 & -10.0 \\
\hline 26 & -686.145275 & -686.387556 & -686.414297 & -685.384619 & -7.0 & -6.8 & -6.1 & 11.4 & -1.9 & -10.2 \\
\hline 26 & -686.143399 & -686.385523 & -686.413507 & -685.382337 & -5.6 & -4.6 & -3.5 & 10.8 & -1.8 & -10.0 \\
\hline 27 & -710.365509 & -710.640572 & -710.660481 & -709.537743 & -8.6 & -8.9 & -9.5 & 14.9 & -2.6 & -11.8 \\
\hline 27 & -710.364143 & -710.640075 & -710.660733 & -709.537494 & -8.4 & -8.3 & -7.3 & 13.7 & -1.8 & -12.8 \\
\hline 27 & -710.361667 & -710.637850 & -710.660086 & -709.535321 & -7.1 & -6.5 & -4.2 & 11.7 & -1.4 & -12.6 \\
\hline 27 & -710.361212 & -710.637372 & -710.659617 & -709.534980 & -6.9 & -5.9 & -4.0 & 11.6 & -1.4 & -12.1 \\
\hline 28 & -710.349682 & -710.622833 & -710.644780 & -709.521274 & -7.8 & -7.6 & -5.9 & 12.6 & -1.7 & -12.6 \\
\hline 28 & -710.349563 & -710.622430 & -710.644201 & -709.521080 & -7.7 & -7.4 & -6.0 & 13.6 & -1.8 & -13.2 \\
\hline 28 & -710.349130 & -710.622100 & -710.644864 & -709.520681 & -7.4 & -7.0 & -5.2 & 15.1 & -2.1 & -14.9 \\
\hline 28 & -710.349615 & -710.622722 & -710.645050 & -709.520316 & -7.2 & -7.0 & -5.5 & 11.8 & -1.6 & -11.6 \\
\hline 28 & -710.348140 & -710.621126 & -710.644327 & -709.519491 & -6.7 & -6.2 & -4.3 & 14.7 & -1.9 & -14.7 \\
\hline 28 & -710.347610 & -710.621029 & -710.644576 & -709.519043 & -6.4 & -5.9 & -3.9 & 11.3 & -1.4 & -11.8 \\
\hline 29 & -710.344554 & -710.617605 & -710.637696 & -709.515524 & -8.1 & -7.8 & -6.4 & 13.0 & -1.6 & -12.8 \\
\hline
\end{tabular}




\begin{tabular}{|c|c|c|c|c|c|c|c|c|c|c|}
\hline 29 & -710.343898 & -710.616612 & -710.637277 & -709.514949 & -7.7 & -7.3 & -5.3 & 14.0 & -1.8 & -14.2 \\
\hline 29 & -710.343513 & -710.616248 & -710.636847 & -709.514365 & -7.4 & -7.0 & -5.5 & 11.9 & -1.4 & -12.1 \\
\hline 30 & -726.413459 & -726.691084 & -726.716072 & -725.583460 & -9.3 & -10.0 & -9.4 & 14.8 & -2.6 & -12.9 \\
\hline 0 & -726.414679 & -726.691495 & -726.714861 & -725.583380 & -9.2 & -9.7 & -10.1 & 15.0 & -2.6 & -12.0 \\
\hline 30 & -726.412436 & -726.690136 & -726.715521 & -725.582166 & -8.4 & -8.1 & -6.1 & 12.8 & -1.7 & -13.0 \\
\hline 30 & -726.412149 & -726.689381 & -726.713765 & -725.581860 & -8.3 & -8.4 & -6.9 & 12.8 & -1.8 & -12.5 \\
\hline 31 & -758.490101 & -758.769660 & -758.798377 & -757.650022 & -11.2 & -11.2 & -10.7 & 15.9 & -2.3 & -14.1 \\
\hline 31 & -758.486981 & -758.766321 & -758.796431 & -757.646763 & -9.1 & -9.5 & -8.6 & 14.2 & -2.3 & -12.8 \\
\hline 31 & -758.486530 & -758.766199 & -758. & -757.646618 & -9.1 & -8.6 & -6.6 & 12.7 & -1.8 & -12.9 \\
\hline 31 & -758.486343 & -758.766003 & -758.796408 & -757.6 & -8.8 & -8.3 & -7.3 & 13.9 & -1.7 & -13.1 \\
\hline 32 & -758.492139 & -758.772145 & -758. & -757 & -9.1 & -9.5 & -9.2 & 14.1 & -1.9 & -12.5 \\
\hline 32 & -758.492187 & -758.772147 & -758 & -757 & -9.1 & -9.1 & -7.9 & 13.2 & -1.6 & -12.8 \\
\hline 32 & -758.491533 & -758.7 & -758.7 & -757.652095 & -8.5 & -8.2 & -7.7 & 13.6 & -1.6 & -12.5 \\
\hline 33 & -767.333672 & -767.612981 & -767.630478 & -766.482391 & -8.3 & -7.7 & -9.1 & 15.2 & -2.5 & -11.4 \\
\hline 33 & -767.331188 & -767.611513 & -767.629601 & -766.481808 & -8.0 & -7.1 & -5.9 & 11.4 & -1.2 & -11.4 \\
\hline 33 & -767.331822 & -767.611624 & -767.6 & -766. & -7.7 & -7.2 & -7.2 & 12.8 & -1.7 & -11.1 \\
\hline 33 & -767.330826 & -767.611244 & -767.629256 & -766.481217 & -7.6 & -6.8 & -5.4 & 11.2 & -1.2 & -11.3 \\
\hline 33 & -767.331218 & -767.611176 & -767.629409 & -766.480730 & -7.3 & -6.7 & -6.6 & 12.3 & -1.6 & -10.8 \\
\hline 33 & -767.3 & -767.6 & -767.6 & -766. & -7.1 & -6.4 & -6.4 & 12.1 & -1.5 & -10.5 \\
\hline 33 & -767.329894 & -767.610104 & -767.628834 & -766.480193 & -6.9 & -6.3 & -4.8 & 11.2 & -1.1 & -11.6 \\
\hline 33 & -767.330407 & -767.610371 & -767.6 & -766.479989 & -6.8 & -6.1 & -5.3 & 11.8 & -1.4 & -11.2 \\
\hline 33 & -767.329186 & -767.609730 & -767.629004 & -766.479760 & -6.7 & -5.5 & -4.1 & 11.1 & -1.1 & -11.4 \\
\hline 34 & -833.735840 & -834.034938 & -834.066752 & -832.831427 & -11.7 & -12.3 & -11.9 & 15.8 & -2.2 & -13.9 \\
\hline 34 & -833.734865 & -834.033911 & -834.065989 & -832.830149 & -10.9 & -10.6 & -10.6 & 15.8 & -1.8 & -14.1 \\
\hline 34 & -833.733228 & -834.032143 & -834.065157 & -832.828265 & -9.7 & -9.2 & -8.8 & 13.0 & -1.7 & -11.7 \\
\hline 34 & -833.732456 & -834.031290 & -834.064254 & -832.827872 & -9.5 & -9.8 & -9.1 & 15.5 & -2.0 & -14.2 \\
\hline
\end{tabular}




\begin{tabular}{|c|c|c|c|c|c|c|c|c|c|c|}
\hline 34 & -833.732147 & -834.030960 & -834.064903 & -832.826485 & -8.6 & -8.6 & -7.9 & 12.9 & -2.0 & -11.5 \\
\hline 35 & -833.732335 & -834.031260 & -834.064234 & -832.828278 & -10.5 & -10.2 & -9.0 & 15.0 & -2.0 & -14.2 \\
\hline 35 & -833.731535 & -834.030417 & -834.064580 & -832.826244 & -9.2 & -9.5 & -8.4 & 13.1 & -2.5 & -11.7 \\
\hline 35 & -833.731105 & -834.029965 & -834.063785 & -832.826082 & -9.1 & -9.0 & -8.6 & 14.1 & -2.1 & -12.3 \\
\hline 35 & -833.731181 & -834.029705 & -834.063890 & -832.825531 & -8.7 & -8.7 & -7.6 & 12.7 & -2.1 & -11.8 \\
\hline 36 & -908.984638 & -909.306188 & -909.336940 & -908.017247 & -11.5 & -11.8 & -11.7 & 15.9 & -2.2 & -13.8 \\
\hline 36 & -908.984022 & -909.305158 & -909.336140 & -908.016682 & -11.2 & -11.4 & -11.9 & 17.5 & -2.1 & -14.9 \\
\hline 37 & -908.969383 & -909.290615 & -909.326627 & -908.002958 & -11.2 & -11.7 & -10.3 & 15.5 & -2.6 & -14.3 \\
\hline 37 & -908.970541 & -909.291427 & -909.327834 & -908.002897 & -11.2 & -12.5 & -11.8 & 16.1 & -3.0 & -13.8 \\
\hline 37 & -908.969286 & -909.290300 & -909.326876 & -908.001602 & -10.4 & -10.3 & -9.3 & 15.2 & -2.4 & -13.8 \\
\hline 38 & -748.455179 & -748.741841 & -748.762137 & -747.581664 & -8.5 & -8.1 & -6.8 & 14.8 & -1.7 & -14.5 \\
\hline 38 & -748.454414 & -748.741388 & -748.762483 & -747.581364 & -8.3 & -7.9 & -5.3 & 13.1 & -1.5 & -14.1 \\
\hline 38 & -748.454485 & -748.740830 & -748.761382 & -747.580578 & -7.8 & -7.6 & -6.7 & 13.7 & -1.8 & -12.8 \\
\hline 38 & -748.453598 & -748.740520 & -748.761544 & -747.580533 & -7.8 & -6.9 & -4.9 & 13.1 & -1.4 & -13.7 \\
\hline 38 & -748.453597 & -748.740228 & -748.761383 & -747.580243 & -7.6 & -6.9 & -4.8 & 13.8 & -1.5 & -14.4 \\
\hline 38 & -748.452991 & -748.739889 & -748.761230 & -747.580051 & -7.5 & -6.7 & -3.9 & 11.9 & -1.2 & -13.5 \\
\hline 39 & -764.490338 & -764.778564 & -764 & -763. & -9.2 & -9.0 & -6.3 & 13.4 & -1.8 & -14.3 \\
\hline 39 & -764.490400 & -764.778224 & -764.802134 & -763.611767 & -8.6 & -8.3 & -7.1 & 14.8 & -1.8 & -14.2 \\
\hline 39 & -764.490244 & -764.777697 & -764.801363 & -763.611375 & -8.4 & -8.4 & -7.9 & 15.0 & -2.1 & -13.4 \\
\hline 39 & -764.489297 & -764.777442 & -764.801707 & -763.611179 & -8.3 & -7.9 & -5.9 & 13.3 & -1.5 & -13.9 \\
\hline 39 & -764.488065 & -764.775362 & -764.800182 & -763.609237 & -7.1 & -6.7 & -5.8 & 13.2 & -1.7 & -12.5 \\
\hline 40 & -764.495085 & -764.783174 & -764.804918 & -763.617611 & -9.3 & -9.9 & -8.8 & 14.8 & -2.0 & -13.9 \\
\hline 40 & -764.495285 & -764.783466 & -764.805743 & -763.617400 & -9.2 & -8.9 & -8.1 & 16.3 & -2.2 & -14.9 \\
\hline 40 & -764.494919 & -764.783429 & -764.805694 & -763.617344 & -9.2 & -8.9 & -7.7 & 15.3 & -1.9 & -14.6 \\
\hline 40 & -764.494981 & -764.782690 & -764.804278 & -763.617229 & -9.1 & -9.3 & -9.2 & 17.3 & -2.2 & -15.2 \\
\hline
\end{tabular}




\begin{tabular}{|c|c|c|c|c|c|c|c|c|c|c|}
\hline 40 & -764.494084 & -764.782566 & -764.805305 & -763.616945 & -8.9 & -8.7 & -6.9 & 14.8 & -1.7 & -14.8 \\
\hline 41 & -769.510796 & -769.801866 & -769.820832 & -768.626765 & -9.1 & -8.9 & -9.2 & 16.2 & -2.8 & -13.0 \\
\hline 41 & -769.508950 & -769.799820 & -769.819287 & -768.625138 & -8.1 & -8.2 & -8.2 & 15.0 & -2.3 & -12.7 \\
\hline 41 & -769.508361 & -769.799991 & -769.819829 & -768.624806 & -7.9 & -7.7 & -7.2 & 14.0 & -1.8 & -12.6 \\
\hline 41 & -769.507512 & -769.799321 & -769.819339 & -768.624543 & -7.7 & -7.3 & -6.1 & 13.9 & -1.7 & -13.4 \\
\hline 41 & -769.507056 & -769.798961 & -769.818979 & -768 & -7.4 & -7.0 & -6.0 & 13.4 & -1.6 & -12.8 \\
\hline 41 & -769.506355 & -769.797857 & -769.817897 & -768 & -7.2 & -6.4 & -4.9 & 12.8 & -1.3 & -13.0 \\
\hline 41 & -769.506274 & -769.798091 & -769.818461 & -768.623428 & -7.0 & -6.7 & -4.6 & 10.9 & -1.2 & -11.7 \\
\hline 41 & -769.506914 & -769.798354 & -769.818089 & -768.623408 & -7.0 & -7.0 & -6.3 & 13.8 & -1.7 & -12.8 \\
\hline 41 & -769.507149 & -769.797989 & -769.8 & -768 & -7.0 & -6.6 & -5.5 & 12.2 & -1.8 & -11.5 \\
\hline 41 & -769.505837 & -769.797338 & -769.817906 & -768.623010 & -6.8 & -6.1 & -4.2 & 11.5 & -1.4 & -11.9 \\
\hline 41 & -769.505720 & -769.797657 & -769.818533 & -768.622982 & -6.8 & -5.9 & -3.7 & 11.1 & -1.2 & -12.1 \\
\hline 41 & -769.50 & -769 & -769.8 & -768.6 & -6.7 & -6.0 & -3.5 & 11.5 & -1.2 & -12.8 \\
\hline 42 & -823 & -823 & -824 & -822 & -10.1 & -9.9 & -8.9 & 15.6 & -2.1 & -14.4 \\
\hline 42 & -823.682446 & -823.988767 & -824.015528 & -822.743322 & -9.8 & -9.7 & -8.2 & 16.3 & -2.1 & -15.8 \\
\hline 42 & -823.681805 & -823.987634 & -824.013704 & -822.742350 & -9.2 & -9.6 & -8.7 & 14.5 & -2.2 & -13.2 \\
\hline 42 & -823 & -823 & -824.015110 & -822.742104 & -9.0 & -8.2 & -5.9 & 14.3 & -1.7 & -15.0 \\
\hline 42 & -823.6 & -823 & -824. & -822. & -8.5 & -7.7 & -5.7 & 14.7 & -1.7 & -15.0 \\
\hline 42 & -823.680334 & -823.986833 & -824.015150 & -822.740660 & -8.1 & -7.3 & -5.5 & 13.0 & -1.5 & -13.3 \\
\hline 42 & -823.679823 & -823.985338 & -824.013804 & -822.739526 & -7.4 & -6.9 & -5.6 & 13.5 & -2.1 & -12.8 \\
\hline 43 & 407 & -864 & -864 & -862 & -11.2 & -11.2 & -9.5 & 17.1 & -2.5 & -16.3 \\
\hline 43 & -863.951816 & -864.292546 & -864.316440 & -862.940816 & -10.3 & -10.3 & -8.0 & 16.6 & -2.4 & -16.5 \\
\hline 43 & -863.950883 & -864.291964 & -864.315474 & -862.940554 & -10.1 & -10.0 & -7.3 & 16.0 & -1.7 & -17.1 \\
\hline 43 & -863.952005 & -864.292525 & -864.315346 & -862.940479 & -10.1 & -10.0 & -8.4 & 16.2 & -2.4 & -15.4 \\
\hline 43 & -863.951430 & -864.292124 & -864.315933 & -862.940353 & -10.0 & -9.9 & -8.2 & 16.9 & -2.3 & -16.3 \\
\hline 43 & -863.951536 & -864.292034 & -864.314874 & -862.939875 & -9.7 & -9.7 & -8.3 & 15.6 & -2.4 & -14.5 \\
\hline 43 & -863.949969 & -864.291577 & -864.315890 & -862.939645 & -9.5 & -9.0 & -6.7 & 15.7 & -1.7 & -16.3 \\
\hline 43 & -863.948453 & -864.289951 & -864.315838 & -862.937986 & -8.5 & -7.9 & -4.9 & 13.7 & -1.5 & -15.1 \\
\hline
\end{tabular}




\begin{tabular}{|c|c|c|c|c|c|c|c|c|c|c|}
\hline 44 & -883.809385 & -884.150181 & -884.171834 & -882.791698 & -9.9 & -9.6 & -7.6 & 15.7 & -2.0 & -15.8 \\
\hline 44 & -883.809637 & -884.150474 & -884.171817 & -882.791431 & -9.7 & -9.6 & -8.5 & 16.7 & -2.1 & -15.8 \\
\hline 44 & -883.808573 & -884.150227 & -884.172206 & -882.791096 & -9.5 & -9.0 & -6.7 & 15.1 & -1.6 & -15.8 \\
\hline 44 & -883.808045 & -884.149313 & -884.171473 & -882.791004 & -9.5 & -9.1 & -6.1 & 14.7 & -1.4 & -16.2 \\
\hline 44 & -883.808900 & -884.149752 & -884.171363 & -882.790694 & -9.3 & -8.8 & -6.9 & 15.0 & -1.9 & -15.0 \\
\hline 44 & -883.808046 & -884.149539 & -884.171610 & -882.790633 & -9.2 & -8.4 & -5.5 & 13.4 & -1.3 & -14.9 \\
\hline 44 & -883.807770 & -884.149047 & -884.171219 & -882.790626 & -9.2 & -8.7 & -5.8 & 14.0 & -1.3 & -15.6 \\
\hline 44 & -883.808606 & -884.149493 & -884.171016 & -882.790600 & -9.2 & -9.0 & -6.9 & 15.9 & -2.2 & -15.7 \\
\hline 44 & -883.807436 & -884.148746 & -884.171106 & -882.789797 & -8.7 & -8.2 & -5.8 & 14.5 & -1.5 & -15.5 \\
\hline 45 & -1206.829131 & $-120^{\circ}$ & -120 & -120 & -10.2 & -10.2 & -8.3 & 16.5 & -2.1 & -16.2 \\
\hline 45 & -1206.828332 & -1207.134474 & -1207.156975 & -1205.413633 & -10.2 & -9.7 & -7.1 & 16.0 & -1.7 & -16.9 \\
\hline 45 & -1206.827753 & -1207.133734 & -1207.156239 & -1205.413528 & -10.1 & -10.2 & -7.0 & 15.3 & -1.7 & -16.8 \\
\hline 45 & -1206.828274 & -1207.133719 & -1207.155908 & -1205.413019 & -9.8 & -9.5 & -7.0 & 16.5 & -2.3 & -16.7 \\
\hline 45 & -1206.827235 & -1207.133210 & -1207.156032 & -1205.412887 & -9.7 & -9.2 & -5.8 & 14.8 & -1.5 & -16.7 \\
\hline 45 & -1206.826964 & -1207.133003 & -1207.155958 & -1205.412753 & -9.6 & -9.3 & -5.7 & 14.7 & -1.5 & -16.7 \\
\hline 45 & -1206.827157 & -1207.133463 & -1207.156385 & -1205.412391 & -9.4 & -8.9 & -5.5 & 14.3 & -1.4 & -16.2 \\
\hline 46 & -923.104732 & -923.464845 & -923.486443 & -922.039250 & -11.4 & -10.8 & -9.8 & 18.7 & -2.6 & -17.2 \\
\hline 46 & -923.104713 & -923.464321 & -923.485399 & -922.038796 & -11.1 & -11.2 & -11.2 & 19.9 & -2.7 & -17.2 \\
\hline 46 & -923.102860 & -923.463401 & -923.485539 & -922.038529 & -10.9 & -10.2 & -8.1 & 17.3 & -1.7 & -17.7 \\
\hline 46 & -923.103992 & -923.463663 & -923.485021 & -922.038367 & -10.8 & -11.1 & -10.3 & 18.6 & -2.8 & -16.7 \\
\hline 46 & -923.103313 & -923.463344 & -923.485628 & -922.037981 & -10.6 & -9.9 & -8.3 & 18.0 & -2.3 & -17.3 \\
\hline 46 & -923.102003 & -923.462594 & -923.485172 & -922.037742 & -10.4 & -9.7 & -7.5 & 17.1 & -1.7 & -17.6 \\
\hline 46 & -923.101864 & -923.462389 & -923.484878 & -922.037052 & -10.0 & -9.0 & -6.7 & 16.2 & -1.6 & -16.9 \\
\hline 46 & -923.101630 & -923.461990 & -923.484747 & -922.036892 & -9.9 & -9.1 & -7.1 & 17.2 & -1.7 & -17.5 \\
\hline 46 & -923.101407 & -923.461638 & -923.484333 & -922.036818 & -9.9 & -9.1 & -6.8 & 15.9 & -1.6 & -16.7 \\
\hline 46 & -923.102250 & -923.461906 & -923.484397 & -922.036596 & -9.7 & -9.3 & -7.5 & 15.6 & -1.9 & -15.5 \\
\hline 46 & -923.101678 & -923.461070 & -923.483396 & -922.036231 & -9.5 & -9.3 & -7.8 & 14.2 & -1.9 & -13.8 \\
\hline
\end{tabular}


Table S8. Absolute energies, binding energies, and SAPT0 energy decomposition for all unique dimers of 1-46 with the model 3methylindole (Trp).

\begin{tabular}{|c|c|c|c|c|c|c|c|c|c|c|}
\hline \multirow{2}{*}{ Het. } & \multirow{2}{*}{$\begin{array}{c}\begin{array}{c}\text { B97D } \\
\text { /def2-TZVPP }\end{array} \\
E\end{array}$} & \multicolumn{2}{|c|}{$\begin{array}{c}\omega B 97 X-D \\
/ \text { def2-TZVPP }\end{array}$} & \multicolumn{2}{|c|}{$\begin{array}{c}\text { DLPNO-CCSD(T) } \\
\text { /cc-pVQZ }\end{array}$} & \multicolumn{5}{|c|}{$\begin{array}{c}\text { SAPT0 } \\
\text { /jun-cc-pVDZ }\end{array}$} \\
\hline & & E (gas) & E (sol) & $\mathbf{E}$ & $\mathbf{E}_{\text {int }}$ & $\mathbf{E}_{\text {total }}$ & $\mathbf{E}_{\text {elec }}$ & $\mathbf{E}_{\text {exch }}$ & $\mathbf{E}_{\text {ind }}$ & $\mathbf{E}_{\text {disp }}$ \\
\hline 1 & -613.084553 & -613.330814 & -613.352689 & -612.362527 & -4.5 & -3.8 & -2.7 & 8.6 & -1.0 & -8.7 \\
\hline 2 & -632.940885 & -633.185941 & -633.204215 & -632.211025 & -5.3 & -4.5 & -3.1 & 9.2 & -1.0 & -9.6 \\
\hline 2 & -632.940733 & -633.185783 & -633.204134 & -632.210487 & -5.0 & -4.3 & -3.0 & 8.5 & -0.9 & -8.9 \\
\hline 3 & -955.964017 & -956.174147 & -956.193030 & -954.835322 & -5.8 & -5.0 & -2.8 & 8.9 & -1.0 & -10.1 \\
\hline 3 & -955.964908 & -956.174601 & -956.192550 & -954.835161 & -5.7 & -5.6 & -4.5 & 8.9 & -1.2 & -8.8 \\
\hline 4 & -648.960470 & -649.201923 & -649.219847 & -648.221655 & -7.2 & -7.7 & -7.3 & 9.8 & -1.6 & -8.6 \\
\hline 4 & -648.959858 & -649.201755 & -649.219643 & -648.221611 & -7.2 & -7.4 & -6.9 & 10.8 & -1.5 & -9.8 \\
\hline 4 & -648.958606 & -649.200409 & -649.219043 & -648.220642 & -6.6 & -6.2 & -5.5 & 9.9 & -1.3 & -9.2 \\
\hline 4 & -648.958864 & -649.200708 & -649.219345 & -648.220289 & -6.3 & -6.4 & -5.7 & 10.4 & -1.5 & -9.6 \\
\hline 4 & -648.958499 & -649.200350 & -649.218942 & -648.220086 & -6.2 & -6.3 & -5.2 & 9.0 & -1.4 & -8.7 \\
\hline 5 & -648.992429 & -649.238644 & -649.256573 & -648.258631 & -6.7 & -6.2 & -5.3 & 10.2 & -1.2 & -9.9 \\
\hline 5 & -648.991917 & -649.238203 & -649.256389 & -648.258091 & -6.4 & -5.7 & -4.7 & 9.7 & -1.1 & -9.5 \\
\hline 5 & -648.992067 & -649.237882 & -649.255708 & -648.257617 & -6.1 & -6.0 & -5.0 & 8.5 & -1.3 & -8.2 \\
\hline 5 & -648.991323 & -649.237618 & -649.256009 & -648.257492 & -6.0 & -5.3 & -4.4 & 9.9 & -1.2 & -9.6 \\
\hline 5 & -648.992387 & -649.237743 & -649.255150 & -648.257417 & -6.0 & -5.7 & -6.0 & 9.0 & -1.3 & -7.5 \\
\hline 5 & -648.991673 & -649.237569 & -649.255253 & -648.257387 & -6.0 & -5.5 & -4.6 & 8.5 & -1.1 & -8.4 \\
\hline 5 & -648.991215 & -649.237096 & -649.255197 & -648.256936 & -5.7 & -5.2 & -4.8 & 9.1 & -1.1 & -8.4 \\
\hline
\end{tabular}




\begin{tabular}{|c|c|c|c|c|c|c|c|c|c|c|}
\hline 6 & -972.008574 & -972.217203 & -972.235400 & -970.872533 & -7.2 & -7.6 & -6.5 & 10.2 & -1.5 & -9.8 \\
\hline 6 & -972.008671 & -972.217074 & -972.235226 & -970.872448 & -7.1 & -7.3 & -6.5 & 10.1 & -1.6 & -9.4 \\
\hline 6 & -972.008421 & -972.216843 & -972.234857 & -970.872343 & -7.1 & -7.3 & -5.7 & 9.6 & -1.4 & -9.8 \\
\hline 6 & -972.007869 & -972.216719 & -972.235164 & -970.872317 & -7.1 & -6.9 & -5.1 & 10.0 & -1.4 & -10.4 \\
\hline 6 & -972.007723 & -972.216581 & -972.235222 & -970.871860 & -6.8 & -6.7 & -5.5 & 11.0 & -1.5 & -10.7 \\
\hline 6 & -972.006842 & -972.215783 & -972.234714 & -970.870912 & -6.2 & -5.9 & -4.6 & 10.0 & -1.3 & -10.0 \\
\hline 7 & -972.010365 & -972.220025 & -972.238054 & -970.876104 & -7.3 & -6.9 & -5.1 & 10.9 & -1.4 & -11.3 \\
\hline 7 & -972.009854 & -972.219574 & -972.238183 & -970.875730 & -7.0 & -6.4 & -4.8 & 11.0 & -1.3 & -11.3 \\
\hline 7 & -972.009599 & -972.219469 & -972.237941 & -970.875460 & -6.9 & -6.2 & -4.6 & 11.2 & -1.4 & -11.4 \\
\hline 7 & -972.010325 & -972.219573 & -972.237518 & -970.875094 & -6.6 & -6.2 & -5.3 & 9.6 & -1.1 & -9.4 \\
\hline 7 & -972.008394 & -972.218056 & -972.237203 & -970.874164 & -6.1 & -5.2 & -2.7 & 8.4 & -1.0 & -9.9 \\
\hline 8 & -629.118475 & -629.364074 & -629.385446 & -628.389788 & -5.1 & -4.7 & -3.5 & 8.6 & -1.1 & -8.7 \\
\hline 9 & -629.139559 & -629.386167 & -629.409137 & -628.412422 & -8.4 & -8.6 & -8.8 & 11.0 & -2.2 & -8.6 \\
\hline 9 & -629.139250 & -629.386183 & -629.409464 & -628.412184 & -8.2 & -8.2 & -8.3 & 10.2 & -1.8 & -8.3 \\
\hline 11 & -645.145436 & -645.389115 & -645.411317 & -644.410665 & -6.0 & -5.5 & -4.6 & 9.0 & -1.3 & -8.6 \\
\hline 12 & -645.152021 & -645.396658 & -645.415222 & -644.417660 & -6.2 & -5.8 & -4.9 & 9.1 & -1.1 & -8.9 \\
\hline 14 & -988.033585 & -988.240488 & -988.258461 & -986.892101 & -9.1 & -9.0 & -7.6 & 11.7 & -1.9 & -11.2 \\
\hline 14 & -988.031610 & -988.238736 & -988.257731 & -986.889953 & -7.8 & -7.4 & -5.9 & 10.4 & -1.6 & -10.3 \\
\hline 14 & -988.031217 & -988.238371 & -988.257537 & -986.889814 & -7.7 & -7.1 & -4.9 & 10.2 & -1.6 & -10.7 \\
\hline 15 & -635.146160 & -635.402989 & -635.422481 & -634.381181 & -6.0 & -5.6 & -4.1 & 10.4 & -1.2 & -10.7 \\
\hline 15 & -635.145630 & -635.402679 & -635.422481 & -634.380986 & -5.9 & -5.1 & -3.4 & 10.4 & -1.2 & -11.0 \\
\hline 16 & -651.183885 & -651.441433 & -651.460668 & -650.414471 & -7.7 & -7.5 & -6.6 & 12.7 & -1.6 & -12.0 \\
\hline 16 & -651.182780 & -651.440680 & -651.460730 & -650.413817 & -7.3 & -6.7 & -5.5 & 13.4 & -1.6 & -13.0 \\
\hline
\end{tabular}




\begin{tabular}{|c|c|c|c|c|c|c|c|c|c|c|}
\hline 16 & -651.183251 & -651.440755 & -651.460162 & -650.413244 & -6.9 & -6.7 & -6.0 & 10.6 & -1.3 & -10.0 \\
\hline 16 & -651.183060 & -651.440570 & -651.460310 & -650.413205 & -6.9 & -6.8 & -5.9 & 10.8 & -1.5 & -10.2 \\
\hline 16 & -651.182639 & -651.440351 & -651.460344 & -650.412739 & -6.6 & -6.4 & -5.0 & 10.6 & -1.3 & -10.6 \\
\hline 16 & -651.181690 & -651.439571 & -651.459878 & -650.412208 & -6.2 & -5.8 & -4.5 & 11.2 & -1.3 & -11.2 \\
\hline 17 & -667.216161 & -667.474398 & -667.493506 & -666.442159 & -8.1 & -7.5 & -6.7 & 14.2 & -1.7 & -13.3 \\
\hline 17 & -667.216086 & -667.473985 & -667.492815 & -666.441468 & -7.7 & -7.4 & -6.8 & 12.0 & -1.5 & -11.1 \\
\hline 17 & -667.215510 & -667.473314 & -667.491997 & -666.441029 & -7.4 & -7.3 & -6.4 & 10.7 & -1.6 & -10.0 \\
\hline 18 & -667.223245 & -667.482338 & -667.503099 & -666.450871 & -8.4 & -8.1 & -7.5 & 14.8 & -2.0 & -13.5 \\
\hline 18 & -667.222648 & -667.482028 & -667.503524 & -666.450377 & -8.1 & -7.8 & -7.0 & 12.3 & -1.6 & -11.6 \\
\hline 18 & -667.222334 & -667.481749 & -667.503536 & -666.449811 & -7.7 & -7.4 & -6.6 & 12.1 & -1.5 & -11.4 \\
\hline 18 & -667.222386 & -667.481564 & -667.503208 & -666.449371 & -7.4 & -7.5 & -7.0 & 12.0 & -1.7 & -10.8 \\
\hline 18 & -667.222346 & -667.481422 & -667.502818 & -666.449254 & -7.4 & -7.2 & -6.9 & 11.5 & -1.5 & -10.3 \\
\hline 18 & -667.221697 & -667.480969 & -667.502910 & -666.449026 & -7.2 & -7.0 & -6.5 & 12.9 & -1.7 & -11.6 \\
\hline 18 & -667.220412 & -667.479748 & -667.501470 & -666.448090 & -6.6 & -6.3 & -4.7 & 10.0 & -1.3 & -10.3 \\
\hline 19 & -667.191167 & -667.446906 & -667.467001 & -666.415262 & -9.5 & -9.4 & -8.9 & 15.2 & -2.3 & -13.4 \\
\hline 19 & -667.189834 & -667.445687 & -667.465866 & -666.413566 & -8.4 & -8.4 & -7.7 & 11.6 & -1.7 & -10.6 \\
\hline 19 & -667.188984 & -667.445088 & -667.466022 & -666.413052 & -8.1 & -7.8 & -6.9 & 13.2 & -1.9 & -12.1 \\
\hline 19 & -667.188963 & -667.445001 & -667.466087 & -666.412834 & -8.0 & -7.8 & -6.9 & 12.6 & -1.9 & -11.7 \\
\hline 20 & -683.264435 & -683.525252 & -683.548777 & -682.488737 & -8.4 & -8.1 & -7.7 & 15.6 & -2.4 & -13.6 \\
\hline 20 & -683.262953 & -683.524450 & -683.548530 & -682.487536 & -7.7 & -7.5 & -6.3 & 11.3 & -1.7 & -10.9 \\
\hline 20 & -683.262394 & -683.523515 & -683.548259 & -682.486933 & -7.3 & -6.4 & -5.1 & 12.5 & -1.9 & -12.1 \\
\hline 21 & -683.226279 & -683.483176 & -683.503133 & -682.446201 & -9.9 & -9.6 & -9.3 & 16.0 & -2.4 & -13.9 \\
\hline 21 & -683.225640 & -683.482243 & -683.502911 & -682.445409 & -9.4 & -9.1 & -8.7 & 15.8 & -2.6 & -13.6 \\
\hline 21 & -683.225608 & -683.482711 & -683.503442 & -682.445386 & -9.4 & -9.3 & -8.9 & 13.4 & -2.0 & -11.7 \\
\hline 21 & -683.225325 & -683.482110 & -683.502522 & -682.445268 & -9.3 & -8.9 & -8.5 & 15.6 & -2.3 & -13.6 \\
\hline 21 & -683.225198 & -683.482046 & -683.502793 & -682.444871 & -9.0 & -8.9 & -8.2 & 13.4 & -2.1 & -11.9 \\
\hline
\end{tabular}




\begin{tabular}{|c|c|c|c|c|c|c|c|c|c|c|}
\hline 21 & -683.223545 & -683.480834 & -683.501384 & -682.443758 & -8.3 & -8.1 & -6.9 & 11.3 & -1.6 & -10.9 \\
\hline 21 & -683.223238 & -683.480091 & -683.501969 & -682.443690 & -8.3 & -7.7 & -6.3 & 13.9 & -2.2 & -13.1 \\
\hline & -683.223569 & -683.480587 & -683.502624 & -682.443623 & -8.2 & -7.8 & -6.4 & 13.0 & -2.2 & -12.2 \\
\hline 21 & -683.223550 & -683.480071 & -683.501676 & -682.443290 & -8.0 & -7.4 & -6.6 & 14.7 & -2.3 & -13.3 \\
\hline 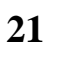 & -683.222843 & -683.479974 & -683.501207 & -682.442925 & -7.8 & -7.4 & -6.1 & 11.7 & -1.7 & -11.3 \\
\hline 21 & -683.223064 & -683.480034 & -683.501772 & -682.442808 & -7.7 & -7.5 & -6.8 & 12.0 & -2.0 & -10.7 \\
\hline 22 & -683.203674 & -683.457440 & -683.476940 & -682. & -10.3 & -10.2 & -9.9 & 16.5 & -2.9 & -13.9 \\
\hline 22 & -683.202313 & -683.456504 & -683.475991 & -682.419817 & -9.5 & -9.4 & -8.7 & 12.7 & -2.1 & -11.3 \\
\hline 22 & -683.201969 & -683.456259 & -683.476322 & -682.419354 & -9.2 & -9.1 & -8.3 & 13.1 & -2.1 & -11.7 \\
\hline 22 & -683.201265 & -683.455657 & -683.476033 & -682.418878 & -8.9 & -8.5 & -7.7 & 13.7 & -2.2 & -12.3 \\
\hline 23 & -699.2 & -699. & -699. & -698. & -9.8 & -9.5 & -10.2 & 18.4 & -3.0 & -14.7 \\
\hline 23 & -699.2 & -699. & -699. & -698. & -9.3 & -9.3 & -8.7 & 13.3 & -2.1 & -11.8 \\
\hline 23 & -699.228262 & -699.481546 & -699.501574 & -698.440853 & -8.9 & -8.9 & -9.0 & 17.6 & -3.2 & -14.3 \\
\hline 24 & -699.240011 & -699.495125 & -699.515437 & -698. & -9.9 & -9.6 & -9.7 & 17.4 & -3.1 & -14.2 \\
\hline 24 & -699.240307 & -699.494874 & -699.515034 & -698.454992 & -9.8 & -9.5 & -9.6 & 17.4 & -3.2 & -14.0 \\
\hline 24 & -699.238753 & 4779 & -699.514633 & & -9.6 & -9.7 & -8.8 & 12.8 & -2.3 & -11.5 \\
\hline 24 & -699.239403 & -699.493909 & -699. & -698. & -9.3 & -9.1 & -8.9 & 17.3 & -3.3 & -14.3 \\
\hline 24 & -699.238926 & -699.493877 & -699.514686 & -698.454081 & -9.2 & -8.8 & -8.7 & 16.9 & -3.1 & -13.9 \\
\hline 24 & -699.238322 & -699.494047 & -699.514562 & -698.453938 & -9.1 & -9.1 & -8.5 & 14.3 & -2.5 & -12.3 \\
\hline 20 & -699.2 & 180 & -699. & -698.422381 & -10.8 & 10.9 & -10.5 & 14.8 & -2.9 & -12.3 \\
\hline 25 & -699.211284 & -699.462303 & -699.480158 & -698.422100 & -10.6 & -10.5 & -10.5 & 18.1 & -3.5 & -14.5 \\
\hline 25 & -699.211181 & -699.462163 & -699.480003 & -698.421866 & -10.5 & -10.6 & -10.2 & 17.6 & -3.7 & -14.4 \\
\hline 26 & -742.458706 & -742.735758 & -742.762181 & -741.617718 & -10.4 & -11.3 & -10.6 & 14.1 & -2.6 & -12.3 \\
\hline 26 & -742.457754 & -742.734745 & -742.762382 & -741.616769 & -9.8 & -9.8 & -8.5 & 14.1 & -2.6 & -12.9 \\
\hline 26 & -742.454722 & -742.731999 & -742.761742 & -741.613872 & -8.0 & -7.7 & -6.0 & 13.4 & -2.3 & -12.8 \\
\hline
\end{tabular}




\begin{tabular}{|c|c|c|c|c|c|c|c|c|c|c|}
\hline 27 & -766.676868 & -766.986419 & -767.007198 & -765.769629 & -11.2 & -11.9 & -10.4 & 17.9 & -3.1 & -16.3 \\
\hline & -766.676876 & -766.986449 & -767.007223 & -765.769621 & -11.2 & -11.9 & -10.4 & 17.9 & -3.1 & -16.3 \\
\hline & -766.675759 & -766.985193 & -767.006942 & -765.768312 & -10.4 & -10.6 & -9.6 & 16.4 & -2.8 & -14.7 \\
\hline 7 & -766.674556 & -766.984301 & -767.006852 & -765.766794 & -9.4 & -9.8 & -8.9 & 12.5 & -2.2 & -11.2 \\
\hline 7 & -766.674099 & -766.983811 & -767.006685 & -765.766791 & -9.4 & -9.5 & -7.6 & 14.5 & -2.5 & -13.8 \\
\hline 27 & -766.671428 & -766.981707 & -767.005320 & -765.764924 & -8.3 & -8.4 & -6.2 & 13.8 & -1.9 & -14.1 \\
\hline 27 & -766.670856 & -766.981888 & -767 & -765.7 & -8.0 & -7.8 & -5.4 & 13.6 & -1.6 & -14.4 \\
\hline 27 & -766.670495 & -766.9 & -767 & -765. & -7.9 & -7.4 & -4.3 & 14.2 & -1.6 & -15.7 \\
\hline 27 & -766.669751 & -766.981026 & -767.0 & -765.763616 & -7.4 & -6.7 & -4.0 & 13.1 & -1.6 & -14.3 \\
\hline 0 & -766. & -766. & -766 & -765 & -10.4 & -11.2 & -10.0 & 18.0 & -3.2 & -16.0 \\
\hline 28 & -766.660983 & -766.968774 & 91647 & -765.752061 & -9.7 & -10.0 & -7.6 & 16.0 & -2.4 & -16.0 \\
\hline 28 & -766.66 & -766.9 & -766 & -765.751934 & -9.6 & -10.1 & -8.6 & 16.0 & -2.7 & -14.8 \\
\hline 28 & -766 . & -766 & -76 & -76 & -9.4 & -9.9 & -8.6 & 16.4 & -2.6 & -15.1 \\
\hline 28 & -766.658134 & -766.966141 & -766.990930 & -765.749613 & -8.2 & -8.2 & -5.9 & 14.2 & -1.8 & -14.8 \\
\hline 28 & -766.6 & -766. & -766 & -765.7 & -7.6 & -7.1 & -3.8 & 14.8 & -1.8 & -16.2 \\
\hline 28 & -766. & -766. & -766 & -765.748729 & -7.6 & -7.6 & -4.8 & 13.9 & -2.1 & -14.6 \\
\hline 28 & -766. & -766. & -766 & -765.748501 & -7.5 & -6.9 & -3.5 & 13.6 & -1.6 & -15.4 \\
\hline 29 & -766.6 & -766 & -766 & -765 & -11.0 & -11.5 & -9.3 & 15.6 & -2.6 & -15.3 \\
\hline 29 & -766.656392 & -766.963408 & -766.984681 & -765.747158 & -10.6 & -11.0 & -8.8 & 17.1 & -2.8 & -16.5 \\
\hline 29 & -766.6 & -766.9 & -766 & -765.745927 & -9.8 & -9.9 & -8.0 & 15.5 & -2.3 & -15.1 \\
\hline 29 & -766.655158 & -766.9 & -766 . & -765.745639 & -9.6 & -9.8 & -8.2 & 16.1 & -2.4 & -15.4 \\
\hline 29 & -766. & -766 & -766 & -765.7 & -8.9 & -8.8 & -7.2 & 14.9 & -2.0 & -14.5 \\
\hline 29 & -766.6 & -766. & -766 & -765.744056 & -8.6 & -8.2 & -5.9 & 14.4 & -2.4 & -14.4 \\
\hline 29 & -766.653158 & -766.960827 & -766.983469 & -765.744000 & -8.6 & -8.6 & -6.5 & 14.6 & -1.9 & -14.8 \\
\hline 29 & -766.653325 & -766.9 & -766.982635 & -765.743798 & -8.5 & -8.2 & -6.0 & 14.1 & -1.9 & -14.3 \\
\hline 29 & -766.652621 & -766.960630 & -766.983519 & -765.743757 & -8.4 & -8.0 & -5.7 & 14.5 & -1.8 & -15.0 \\
\hline 29 & -766.653110 & -766.960682 & -766.983392 & -765.743755 & -8.4 & -8.4 & -6.5 & 14.2 & -1.8 & -14.4 \\
\hline 29 & -766.652290 & -766.960170 & -766.983274 & -765.743504 & -8.3 & -7.6 & -5.0 & 14.5 & -1.7 & -15.5 \\
\hline 29 & -766.652255 & -766.959823 & -766.983091 & -765.743471 & -8.3 & -7.6 & -4.7 & 14.4 & -1.8 & -15.6 \\
\hline
\end{tabular}




\begin{tabular}{|c|c|c|c|c|c|c|c|c|c|c|}
\hline 29 & -766.652179 & -766.959612 & -766.982786 & -765.743101 & -8.0 & -7.4 & -4.4 & 13.6 & -1.4 & -15.2 \\
\hline 29 & -766.651554 & -766.959063 & -766.982409 & -765.742164 & -7.4 & -7.0 & -4.8 & 13.0 & -1.6 & -13.7 \\
\hline 30 & -782.726335 & -783.037735 & -783.062027 & -781.815253 & -11.8 & -12.5 & -10.5 & 16.9 & -3.2 & -15.6 \\
\hline 30 & -782.726336 & -783.037732 & -783.062027 & -781.815250 & -11.8 & -12.5 & -10.5 & 16.9 & -3.2 & -15.6 \\
\hline 30 & -782.725732 & -783.037762 & -783.062559 & -781.814900 & -11.6 & -12.1 & -10.5 & 17.6 & -3.0 & -16.2 \\
\hline 30 & -782.725538 & -783.037304 & -783.062519 & -781.814278 & -11.2 & -11.4 & -9.2 & 15.1 & -2.9 & -14.5 \\
\hline 30 & -782.722448 & -783.034465 & -783.061007 & -781.812149 & -9.9 & -9.8 & -7.5 & 15.1 & -2.0 & -15.3 \\
\hline 30 & -782.721321 & -783.033521 & -783.060855 & -781.811178 & -9.3 & -8.9 & -6.8 & 15.3 & -1.8 & -15.6 \\
\hline 30 & -782.721314 & -783.033436 & -783.059967 & -781.811177 & -9.3 & -9.5 & -7.3 & 15.0 & -2.5 & -14.7 \\
\hline 30 & -782.7 & -783 & -783 & -781 & -8.1 & -7.1 & -4.4 & 13.8 & -1.5 & -15.0 \\
\hline 31 & -814.802172 & -815 & -815 & -813 & -13.6 & -14.3 & -13.2 & 19.3 & -3.6 & -16.9 \\
\hline 31 & -814.80 & -815.1 & -815 . & -813.881478 & -13.5 & -14.3 & -13.0 & 19.4 & -3.8 & -16.9 \\
\hline 31 & -814.800343 & -815.113502 & -815.144424 & -813.878791 & -11.9 & -12.4 & -10.9 & 14.6 & -3.5 & -12.6 \\
\hline 31 & -814.7 & -815.112230 & -815.143684 & -813 & -11.4 & -11.6 & -10.1 & 16.1 & -3.2 & -14.4 \\
\hline 31 & -814.79 & -815 & -815 . & -813 & -11.3 & -11.8 & -10.3 & 17.7 & -3.2 & -16.0 \\
\hline 31 & -814.7 & -815.1 & -815 . & -813 & -10.3 & -10.1 & -6.9 & 13.5 & -2.2 & -14.4 \\
\hline 32 & -814.805416 & -815.1 & -815.1 & -813. & -12.4 & -12.5 & -11.3 & 17.3 & -2.9 & -15.7 \\
\hline 32 & -814.802398 & -815.116802 & -815.145925 & -813.883204 & -10.6 & -10.5 & -9.1 & 17.1 & -2.2 & -16.2 \\
\hline 32 & -814.801109 & -815.115584 & -815.144598 & -813.881987 & -9.8 & -10.0 & -7.7 & 14.5 & -2.3 & -14.4 \\
\hline 32 & -814.800543 & -815.115637 & -815.14 & -813 & -9.8 & -9.4 & -7.4 & 15.7 & -1.8 & -16.0 \\
\hline 32 & -814.799726 & -815.114865 & -815.144846 & -813.880932 & -9.2 & -8.2 & -5.4 & 13.2 & -1.4 & -14.6 \\
\hline 32 & -814.799429 & -815.114228 & -815.145325 & -813.880845 & -9.1 & -8.1 & -5.0 & 14.0 & -1.8 & -15.3 \\
\hline 32 & -814.800087 & -815.114689 & -815.144840 & -813.880831 & -9.1 & -8.5 & -6.5 & 13.7 & -1.7 & -14.0 \\
\hline 32 & -814.799582 & -815.114374 & -815.145055 & -813.880036 & -8.6 & -8.7 & -6.6 & 13.4 & -1.7 & -13.8 \\
\hline 33 & -823.643927 & -823.957667 & -823.976533 & -822.713109 & -10.2 & -9.7 & -9.4 & 16.5 & -2.8 & -14.0 \\
\hline 33 & -823.642403 & -823.957032 & -823.976686 & -822.711552 & -9.2 & -8.7 & -7.5 & 14.0 & -1.9 & -13.4 \\
\hline 33 & -823.642483 & -823.956341 & -823.975790 & -822.711271 & -9.1 & -8.4 & -8.0 & 14.6 & -2.4 & -12 \\
\hline
\end{tabular}




\begin{tabular}{|c|c|c|c|c|c|c|c|c|c|c|}
\hline 33 & -823.642082 & -823.955853 & -823.975707 & -822.710738 & -8.7 & -8.1 & -7.9 & 14.2 & -2.3 & -12.0 \\
\hline 33 & -823.641271 & -823.955911 & -823.976040 & -822.710336 & -8.5 & -7.7 & -6.1 & 12.5 & -1.7 & -12.3 \\
\hline 33 & -823.638971 & -823.953876 & -823.974420 & -822.710189 & -8.4 & -8.0 & -5.6 & 13.5 & -1.6 & -14.2 \\
\hline 33 & -823.638671 & -823.954056 & -823.975454 & -822.710096 & -8.3 & -7.6 & -4.4 & 12.4 & -1.4 & -14.1 \\
\hline 33 & -823.638937 & -823.954392 & -823.975544 & -822.710018 & -8.3 & -7.6 & -5.1 & 13.2 & -1.3 & -14.3 \\
\hline 33 & -823.639007 & -823.953680 & -823.974007 & -822.709775 & -8.1 & -8.0 & -6.4 & 12.5 & -1.5 & -12.6 \\
\hline 3 & -823.638915 & -823.954235 & -823.974952 & -822.709313 & -7.8 & -6.8 & -4.6 & 12.4 & -1.4 & -13.3 \\
\hline 33 & -823.639148 & -823.954254 & -823.975050 & -822.709063 & -7.7 & -6.8 & -5.0 & 13.0 & -1.5 & -13.3 \\
\hline 33 & -823.638059 & -823.953645 & -823.975014 & -822.708647 & -7.4 & -6.8 & -4.1 & 12.4 & -1.3 & -13.9 \\
\hline 34 & -890. & -890 & -89 & 784 & -14.0 & -14.2 & -13.8 & 21.3 & -3.2 & -18.5 \\
\hline 34 & -890.047022 & -890.380773 & -890.413853 & -889.062151 & -13.6 & -13.8 & -12.9 & 19.9 & -3.0 & -17.8 \\
\hline 34 & -890.047676 & -890.380146 & -890.412372 & -889.061963 & -13.5 & -14.0 & -13.3 & 18.5 & -3.3 & -15.8 \\
\hline 34 & -890.045847 & -890.378927 & -890.412028 & -889.060208 & -12.4 & -13.1 & -12.0 & 16.9 & -2.8 & -15.3 \\
\hline 34 & -890.043812 & -890.376934 & -890.411478 & -889.059149 & -11.7 & -11.7 & -10.0 & 16.9 & -3.0 & -15.6 \\
\hline 34 & -890.0 & -890 & -890 & -889 & -11.7 & -11.3 & -9.3 & 16.5 & -2.3 & -16.3 \\
\hline 34 & -890.044234 & -890.377116 & -890.411174 & -889.058780 & -11.5 & -12.0 & -10.5 & 15.7 & -2.7 & -14.4 \\
\hline 34 & -890.043539 & -890.376840 & -890.411191 & -889.058748 & -11.5 & -11.9 & -10.5 & 16.7 & -2.8 & -15.3 \\
\hline 34 & -890.043134 & -890.376898 & -890.411791 & -889.058615 & -11.4 & -11.1 & -8.9 & 16.0 & -2.2 & -16.0 \\
\hline 35 & -890 & -890 & -890 & -885 & -14.4 & -15.2 & -13.8 & & -3.2 & -17.1 \\
\hline 35 & -890.045622 & -890 & -890.412066 & -889.061010 & -13.6 & -14.2 & -13.1 & 19.3 & -2.8 & -17.6 \\
\hline 35 & -890.044801 & -890.377839 & -890.411797 & -889.059829 & -12.9 & -13.5 & -12.2 & 16.7 & -3.0 & -15.0 \\
\hline 35 & -890.043496 & -890.377265 & -890.411822 & -889.058788 & -12.2 & -12.5 & -10.7 & 17.9 & -3.2 & -16.5 \\
\hline 35 & -890.043362 & -890.376356 & -890.410339 & -889.058481 & -12.0 & -12.8 & -10.7 & 15.7 & -2.9 & -14.9 \\
\hline 35 & -890.043429 & -890.3 & -890.4 & -889.057559 & -11.5 & -11.6 & -10.5 & 17.4 & -2.8 & -15.8 \\
\hline 35 & -890.041302 & -890.375078 & -890.410741 & -889.057050 & -11.1 & -11.0 & -8.7 & 16.3 & -2.7 & -15.9 \\
\hline 35 & -890.039942 & -890.373932 & -890.410129 & -889.055680 & -10.3 & -10.2 & -7.7 & 15.3 & -2.0 & -15.8 \\
\hline 36 & -965.297763 & -965.652933 & 83869 & -964.249242 & -14.2 & -15.2 & -14.6 & 19.9 & -3.5 & -17.1 \\
\hline 36 & -965.296046 & -965.651747 & -965.683825 & -964.248406 & -13.7 & -14.3 & -13.3 & 18.7 & -2.8 & -16.8 \\
\hline
\end{tabular}




\begin{tabular}{|c|c|c|c|c|c|c|c|c|c|c|}
\hline 36 & -965.296235 & -965.651957 & -965.684158 & -964.248326 & -13.6 & -14.2 & -13.4 & 20.5 & -3.2 & -18.0 \\
\hline 36 & -965.294871 & -965.651031 & -965.683166 & -964.247627 & -13.2 & -13.4 & -12.2 & 17.6 & -2.4 & -16.4 \\
\hline 36 & -965.294074 & -965.650046 & -965.682648 & -964.246559 & -12.5 & -12.5 & -11.5 & 17.5 & -2.3 & -16.2 \\
\hline 36 & -965.294129 & -965.649362 & -965.682068 & -964.245929 & -12.1 & -12.3 & -11.6 & 17.5 & -2.9 & -15.2 \\
\hline 36 & -965.293204 & -965.649548 & -965.682587 & -964.245658 & -12.0 & -11.7 & -10.3 & 17.2 & -2.0 & -16.6 \\
\hline 36 & -965.293369 & -965.649040 & -965.682523 & -964.245616 & -11.9 & -12.3 & -10.7 & 17.0 & -3.1 & -15.5 \\
\hline 36 & -965.293227 & -965.649363 & -965.683187 & -964.245527 & -11.9 & -11.7 & -10.1 & 16.7 & -2.2 & -16.2 \\
\hline 36 & -965.292402 & -965.648895 & -965.683163 & -964.245408 & -11.8 & -10.9 & -9.2 & 17.2 & -2.1 & -16.9 \\
\hline 36 & -965.292130 & -965.648552 & -965.682679 & -964.244870 & -11.5 & -10.5 & -9.0 & 16.4 & -1.7 & -16.1 \\
\hline 36 & -965.293175 & -965.648574 & -965.6 & -964.244587 & -11.3 & -11.3 & -10.1 & 14.5 & -2.3 & -13.5 \\
\hline 36 & -965.291988 & -965.648030 & -965.682478 & -964.244225 & -11.1 & -10.9 & -9.2 & 16.6 & -2.2 & -16.1 \\
\hline 36 & -965.291999 & -965.648195 & -965.682155 & -964.244109 & -11.0 & -10.7 & -9.3 & 17.1 & -2.1 & -16.4 \\
\hline 36 & -965 & -965 & -96 & -964 & -10.6 & -10.1 & -8.9 & 14.0 & -2.0 & -13.2 \\
\hline 36 & -965.290883 & -965.646577 & -965.681407 & -964.242354 & -9.9 & -9.8 & -8.9 & 14.3 & -2.0 & -13.2 \\
\hline 37 & -965.285682 & -965.641135 & -965.675878 & -964.237861 & -15.8 & -17.3 & -16.2 & 21.8 & -4.4 & -18.5 \\
\hline 37 & -965.284002 & -965.639969 & -965.675745 & -964.236700 & -15.0 & -15.8 & -14.7 & 19.6 & -3.5 & -17.1 \\
\hline 37 & -965.282061 & -965.6 & -965 & -964 & -13.6 & -15.0 & -13.3 & 18.1 & -3.3 & -16.4 \\
\hline 37 & -965.280605 & -965.636876 & -965.675388 & -964.233935 & -13.3 & -13.0 & -11.4 & 19.1 & -2.9 & -17.8 \\
\hline 37 & -965.280632 & -965.635526 & -965.674075 & -964.232416 & -12.3 & -13.1 & -10.5 & 17.1 & -4.2 & -15.5 \\
\hline 37 & -965.278935 & -965.635167 & -965. & -964.231945 & -12.0 & -11.7 & -9.7 & 17.3 & -2.8 & -16.5 \\
\hline 37 & -965.276322 & -965.632978 & -965.672985 & -964.230464 & -11.1 & -10.7 & -8.1 & 16.1 & -2.4 & -16.3 \\
\hline 37 & -965.276332 & -965.631884 & -965.672532 & -964.228956 & -10.2 & -9.8 & -8.4 & 14.1 & -2.2 & -13.3 \\
\hline 38 & -804.765705 & -805.087388 & -805.109257 & -803.812657 & -10.5 & -10.4 & -8.3 & 17.4 & -2.1 & -17.4 \\
\hline 38 & -804.765363 & -805.086612 & -805.108697 & -803.812390 & -10.4 & -10.4 & -7.6 & 16.9 & -2.4 & -17.4 \\
\hline 38 & -804.765806 & -805.086999 & -805.108858 & -803.812359 & -10.4 & -10.9 & -8.8 & 16.6 & -2.3 & -16.4 \\
\hline 38 & -804.765122 & -805.086587 & -805.109137 & -803.812267 & -10.3 & -10.2 & -7.8 & 18.0 & -2.2 & -18.2 \\
\hline 38 & -804.765239 & -805.086249 & -805.108345 & -803.811945 & -10.1 & -10.4 & -8.5 & 17.4 & -2.6 & -16.6 \\
\hline 38 & -804.765264 & -805.086182 & -805.108079 & -803.811913 & -10.1 & -10.1 & -8.0 & 17.3 & -2.0 & -17.4 \\
\hline 38 & -804.764806 & -805.086621 & -805.109082 & -803.811723 & -10.0 & -9.6 & -7.0 & 16.3 & -1.9 & -17.0 \\
\hline
\end{tabular}




\begin{tabular}{|c|c|c|c|c|c|c|c|c|c|c|}
\hline 38 & -804.765286 & -805.086269 & -805.108499 & -803 & -9.9 & -10.1 & -7.7 & 16.0 & -2.2 & -16.2 \\
\hline 38 & -804.764818 & -805.085884 & -805.108398 & -803.811614 & -9.9 & -9.7 & -6.6 & 16.3 & -2.2 & -17.2 \\
\hline 38 & -804.765084 & -805.086174 & -805.108184 & -803.811607 & -9.9 & -10.1 & -8.0 & 16.3 & -2.1 & -16.3 \\
\hline - & -804 & -805 & 500 & 1460 & -9.8 & -9.6 & -6.6 & 16.0 & -2.2 & -16.8 \\
\hline 38 & -804.764746 & 305.085911 & 05.108332 & 11307 & -9.7 & -9.6 & -7.2 & 16.3 & -2.1 & -16.6 \\
\hline 38 & -804.764646 & -805.086189 & -805.108755 & -803.811265 & -9.7 & -9.8 & -7.7 & 16.0 & -2.0 & -16.0 \\
\hline 38 & -804.764005 & -805.085514 & 08518 & 148 & -9.6 & -9.1 & -6.5 & 17.1 & -2.0 & -17.7 \\
\hline 38 & -804.763931 & -805.085635 & -805.108521 & -803.811073 & -9.5 & -9.5 & -7.3 & 17.9 & -2.2 & -18.0 \\
\hline 38 & -804.763959 & -805.085459 & -805.108596 & -803.810999 & -9.5 & -9.2 & -6.3 & 17.0 & -2.0 & -17.9 \\
\hline 38 & -804 & 428 & 476 & -80 & -9.3 & -8.7 & -5.8 & 15.7 & -1.8 & -16.8 \\
\hline 38 & -804.763939 & -805.085439 & -805.108209 & -803.810534 & -9.2 & -9.1 & -6.8 & 15.1 & -1.8 & -15.6 \\
\hline 38 & -804.762658 & -805.084185 & -805.107818 & -803.809999 & -8.9 & -8.2 & -4.9 & 15.6 & -1.8 & -17.1 \\
\hline 38 & -804 & -805 & 8101 & 788 & -8.7 & -8.2 & -4.3 & 13.6 & -1.4 & -16.1 \\
\hline 38 & -804.763076 & -805.084936 & -805.108117 & -803.809750 & -8.7 & -8.6 & -6.0 & 14.1 & -1.7 & -15.1 \\
\hline 38 & -804.762645 & -805 & 721 & -803 & -8.7 & -8.4 & -5.9 & 16.2 & -1.8 & -16.9 \\
\hline 38 & -804.7 & 1495 & 929 & -803 & -8.7 & -8.2 & -5.1 & 14.2 & -1.6 & -15.8 \\
\hline 38 & -804.762264 & -805.084229 & -805.107847 & 09562 & -8.6 & -8.0 & -4.0 & 13.6 & -1.4 & -16.2 \\
\hline 38 & -804.762464 & -805.084098 & 7586 & 9179 & -8.4 & -7.7 & -4.6 & 13.8 & -1.5 & -15.5 \\
\hline 38 & -804.762536 & 3408 & 6694 & 3929 & -8.2 & -7.9 & -5.8 & 14.3 & -1.8 & -14.6 \\
\hline 38 & -804.762023 & -805.083406 & -805.107273 & 8915 & -8.2 & -7.7 & -4.9 & 14.7 & -1.9 & -15.6 \\
\hline 38 & -804.762141 & -805.083450 & -805.107225 & -803.808841 & -8.1 & -7.5 & -4.5 & 15.0 & -1.7 & -16.3 \\
\hline 39 & -820.802753 & -821.125172 & -821.149584 & -819.844547 & -11.8 & -12.2 & -10.2 & 18.3 & -2.5 & -17.8 \\
\hline 39 & -820.802464 & -821.124316 & -821.148259 & -819.844233 & -11.6 & -12.3 & -10.3 & 17.7 & -2.7 & -17.0 \\
\hline 39 & -820.800856 & -821.123246 & -821.148953 & -819.843082 & -10.9 & -10.9 & -8.7 & 19.4 & -2.7 & -18.9 \\
\hline 39 & -820.801264 & -821.123699 & -821.149083 & -819.842882 & -10.8 & -11.2 & -8.9 & 16.3 & -2.2 & -16.3 \\
\hline 39 & -820.800138 & -821.122612 & -821.148165 & -819.842146 & -10.3 & -10.2 & -7.2 & 16.7 & -2.4 & -17.3 \\
\hline 39 & -820.800139 & -821.122835 & -821.148833 & -819.842094 & -10.3 & -10.4 & -8.7 & 19.1 & -2.5 & -18.4 \\
\hline 39 & -820.799697 & -821.122269 & -821.147971 & -819.841880 & -10.2 & -10.0 & -7.1 & 16.7 & -2.2 & -17.3 \\
\hline 39 & -820.799890 & -821.122571 & -821.148614 & -819.841559 & -10.0 & -10.0 & -8.0 & 15.9 & -2.1 & -15.9 \\
\hline 39 & -820.797665 & -821.120808 & -821.147653 & -819.840153 & -9.1 & -8.8 & -5.0 & 14.0 & -1.7 & -16.1 \\
\hline
\end{tabular}




\begin{tabular}{|c|c|c|c|c|c|c|c|c|c|c|}
\hline 40 & -820.805019 & -821.127751 & -821.151507 & -819.847507 & -10.7 & -10.5 & -8.6 & 17.7 & -2.0 & -17.5 \\
\hline 40 & -820.805023 & -821.128015 & -821.151917 & -819.847498 & -10.7 & -11.1 & -9.3 & 16.6 & -2.2 & -16.3 \\
\hline 41 & 820.804867 & -821.127786 & -821.152142 & -819.847389 & -10.6 & -10.5 & -7.8 & 17.5 & -2.3 & -17.8 \\
\hline 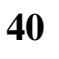 & -820. & -821.128226 & 178 & & -10.6 & -11.0 & -8.9 & 16.3 & -2.2 & 16.2 \\
\hline $\mathbf{0}$ & 820.8 & 21.128040 & -821 & 7225 & -10.5 & -10.5 & 8.4 & 17.7 & -2.2 & -17.5 \\
\hline 40 & -820.804661 & -821.127493 & -821.151373 & -819 . & -10.5 & -10.5 & -8.5 & 16.9 & -2.0 & -16.9 \\
\hline 40 & -820.804971 & -821.127961 & -821.151639 & -819.847165 & -10.5 & -10.9 & -9.0 & 15.8 & -2.2 & -15.6 \\
\hline 40 & 831 & 8008 & -821. & -81 & -10.5 & -10.2 & -8.0 & 17.2 & -2.0 & -17.4 \\
\hline 40 & -820 & -821.127461 & -821 & -81 & -10.4 & -10.6 & -8.6 & 18.2 & -2.4 & -17.8 \\
\hline 40 & -820 & 743 & -821 & -8 & -10.4 & -10.7 & -9.1 & 18.5 & -2.7 & -17.4 \\
\hline 40 & 76 & 146 & -821 & -81 & -10.3 & -10.1 & -8.2 & 18.9 & -2.3 & -18.5 \\
\hline 40 & -820.803836 & -821.127432 & -821. & -819 & -10.2 & -9.9 & -6.5 & 14.5 & -1.6 & -16.4 \\
\hline 40 & -820 . & -821. & -821 & -81 & -10.1 & -10.0 & -7.6 & 17.3 & -2.2 & -17.6 \\
\hline 40 & -820. & -821.126719 & -821 & -819 & -10.0 & -9.5 & -7.2 & 17.4 & -2.0 & -17.7 \\
\hline 40 & -820 . & -821 & -821. & -81 & -9.9 & -9.9 & -7.2 & 17.2 & -2.4 & -17.5 \\
\hline 40 & -820 & 6726 & -821 & -81 & -9.8 & -9.4 & -6.2 & 15.1 & -1.9 & -16.5 \\
\hline 40 & -820 & -821.126068 & -821 & -81 & -9.8 & -9.6 & -6.6 & 16.2 & -2.3 & -16.9 \\
\hline 40 & -820 . & 5912 & -821 & -81 & -9.7 & -9.5 & -6.9 & 17.8 & -2.3 & -18.1 \\
\hline 40 & -820 & 6900 & -821 & -8 & -9.7 & -9.8 & -7.7 & 16.8 & -2.2 & -16.7 \\
\hline 40 & -820 & -821 & -821 & -81 & -9.6 & -9.5 & -7.7 & 18.1 & -2.2 & -17.7 \\
\hline 40 & -820 & -821.126339 & -821 & -8 & -9.3 & -9.3 & -7.1 & 14.8 & -1.8 & -15.2 \\
\hline 40 & -820 & -821 & -821 & -81 & -9.3 & -8.6 & -5.9 & 15.6 & -1.9 & -16.5 \\
\hline 40 & -820.8 & -821.125506 & -821.150402 & -819 & -9.3 & -9.3 & -7.2 & 16.5 & -2.5 & -16.2 \\
\hline 40 & -820.802335 & -821.126010 & -821.151337 & -819.845138 & -9.2 & -9.1 & -6.7 & 15.8 & -1.8 & -16.4 \\
\hline 40 & -820.801995 & -821.125173 & -821.150315 & -819.845045 & -9.2 & -8.5 & -5.3 & 14.7 & -1.6 & -16.2 \\
\hline . & 32 & 93 & 77 & & -10.9 & -10.8 & -9.4 & 17.3 & -2.9 & -15 \\
\hline 41 & -825.820742 & -826.146025 & -826.166610 & -824 & -10.6 & -10.4 & -9.1 & 17.2 & -2.9 & -15.6 \\
\hline 41 & -825.818943 & -826.144782 & -826 & -824 & -10.0 & -10.3 & -8.9 & 17.6 & -2.7 & -16.3 \\
\hline 41 & -825.818780 & -826.144547 & -826.165492 & -824.855294 & -9.7 & -10.1 & -8.4 & 15.8 & -2.3 & -15 \\
\hline
\end{tabular}




\begin{tabular}{|c|c|c|c|c|c|c|c|c|c|c|}
\hline 41 & -825.819703 & -826.144931 & -826.166053 & -824.855288 & -9.7 & -9.3 & -8.1 & 15.2 & -2.6 & -13.7 \\
\hline 11 & -825.819265 & -826.145387 & -826.166775 & -824.855260 & -9.6 & -9.6 & -7.8 & 15.8 & -2.1 & -15.4 \\
\hline & 325.817664 & 26.144300 & 826.165861 & -824.8 & -9.5 & -9.1 & -6.9 & 16.6 & -1.9 & -16.9 \\
\hline 41 & -825.818562 & -826.144155 & -826.165086 & -824.854927 & -9.4 & -9.8 & -9.0 & 14.9 & -2.3 & -13.5 \\
\hline 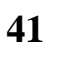 & -825.817163 & 826.143365 & -826.165019 & -824.854849 & -9.4 & -9.2 & -6.6 & 15.1 & -1.9 & -15.7 \\
\hline - & 825.8 & -826.1 & -826.1 & -824 & -9.3 & -9.2 & -7.6 & 15.7 & -2.2 & -15.2 \\
\hline 41 & -825.8 & -826 & -826 & -824 & -9.3 & -9.3 & -7.2 & 15.1 & -2.0 & -15.2 \\
\hline 41 & -825.8 & 124 & -826. & -824 & -9.3 & -9.8 & -8.7 & 15.1 & -2.3 & -14.0 \\
\hline 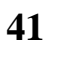 & -825 & -826. & -826. & 630 & -9.2 & -9.0 & -6.3 & 15.2 & -2.1 & -15.8 \\
\hline 41 & -825.817127 & -826.143581 & -826.165111 & -824.854540 & -9.2 & -9.0 & -7.5 & 14.3 & -1.8 & -14.1 \\
\hline 41 & -825 & -826.1 & -826 & -824 & -9.2 & -9.3 & -7.4 & 15.3 & -2.1 & -15.1 \\
\hline 41 & -825. & -826.143320 & -826. & -824 & -9.0 & -8.5 & -6.5 & 16.9 & -2.0 & -16.9 \\
\hline 41 & -825 . & -826.1 & -826.1 & -824 & -8.9 & -8.7 & -6.8 & 14.8 & -1.9 & -14.9 \\
\hline 41 & -825 & -826. & -826. & 92 & -8.9 & -8.9 & -6.8 & 14.6 & -2.1 & -14.6 \\
\hline 41 & -825.816398 & -826.142474 & -826.16 & -824 & -8.9 & -8.8 & -6.5 & 13.7 & -1.6 & -14.5 \\
\hline 41 & -825 . & -826 & -826 & -824 & -8.8 & -8.8 & -6.2 & 12.6 & -1.7 & -13.5 \\
\hline 41 & -825 & -826. & -826 & -82 & -8.8 & -7.8 & -5.2 & 16.1 & -1.9 & -16.8 \\
\hline 41 & -825.8 & -826.1 & -826.1 & -824 & -8.6 & -7.8 & -4.6 & 14.0 & -1.7 & -15.6 \\
\hline 1 & -825 & -826 & -826. & -824 & -8.5 & -8.1 & -5.6 & 14.7 & -1.8 & -15.4 \\
\hline 41 & -825. & -826 & -826 & -824 & -8.5 & -8.7 & -7.0 & 15.7 & -2.1 & -15.3 \\
\hline 41 & -825.8 & -826.1 & -826. & -824 & -7.6 & -7.7 & -6.3 & 13.6 & -2.0 & -13.1 \\
\hline 1 & -825.815190 & -826.14 & -826.1 & 175 & -7.1 & -6.5 & -4.0 & 11.0 & -1.7 & -11.8 \\
\hline 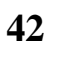 & -018 & 244 & -000 & $-0 / 0$ & 13.3 & 14.7 & 13.2 & 21.1 & -3.4 & -19.2 \\
\hline 42 & -879.9 & -880.3 & -880 & -878 & -13.2 & -14.4 & -12.6 & 20.9 & -3.6 & -19.0 \\
\hline 42 & -879.996093 & -880.336145 & -880.3 & -878 & -13.1 & -14.1 & -13.0 & 20.5 & -3.4 & -18.3 \\
\hline 42 & -879 & -880 & -880 & -87 & -12.6 & -14.1 & -13.1 & 19.2 & -3.3 & -16.9 \\
\hline 42 & -879.995775 & -880.3 & -880 & -878 & -12.4 & -13.2 & -11.5 & 18.9 & -3.3 & -17.3 \\
\hline 42 & -879.994951 & -880.3 & -880.362927 & -878.975173 & -12.4 & -13.1 & -11.5 & 19.5 & -2.9 & -18.2 \\
\hline 42 & -879.993797 & 085 & -880.363530 & 74534 & -12.0 & -12.2 & -9.7 & 18.8 & -2.7 & -18.7 \\
\hline 42 & -879.994343 & -880.334570 & -880.361417 & -878.973834 & -11.5 & -12.7 & -11.8 & 17.3 & -2.6 & -15.6 \\
\hline
\end{tabular}




\begin{tabular}{|c|c|c|c|c|c|c|c|c|c|c|}
\hline 42 & -879.992995 & -880.333740 & -880.362220 & -878.973365 & -11.2 & -11.5 & -9.7 & 17.7 & -2.9 & -16.7 \\
\hline 12 & -879.991707 & -880.332882 & -880.362117 & -878.972483 & -10.7 & -10.2 & -7.6 & 17.3 & -2.3 & -17.7 \\
\hline 42 & -879.992175 & -880.332615 & -880.361930 & -878.972434 & -10.7 & -10.9 & -8.1 & 17.3 & -3.1 & -17.0 \\
\hline (n) & -879.990948 & -880.3 & -880 & -878 & -10.1 & -10.4 & -7.6 & 16.6 & -2.7 & -16.7 \\
\hline 42 & -879.990190 & -880.331255 & -880.361335 & -878.971520 & -10.1 & -9.5 & -6.7 & 17.5 & -2.3 & -18.0 \\
\hline 42 & -879.989500 & -880.330561 & -880.360376 & -878.970477 & -9.4 & -9.1 & -6.5 & 16.8 & -2.4 & -17.1 \\
\hline 42 & -879.988865 & -880.329772 & -880.360397 & -878.969403 & -8.8 & -8.2 & -5.5 & 15.8 & -1.9 & -16.6 \\
\hline 45 & -920.265759 & -920. & -920.6 & 89 & -14.2 & -14.8 & -12.1 & 1.5 & -3.3 & -20.8 \\
\hline 43 & -920.261682 & -920. & -920.6 & 133 & -11.9 & -12.2 & -8.7 & 18.9 & -2.5 & -20.0 \\
\hline 43 & -920.261404 & -920.636992 & -920.662270 & -919.170624 & -11.6 & -11.9 & -9.2 & 18.8 & -2.7 & -18.7 \\
\hline 43 & -920.262026 & -920.6 & -920.6 & 0493 & -11.5 & -11.7 & -9.3 & 17.4 & -3.1 & -16.8 \\
\hline 44 & -940.120072 & -940 & -940. & -939 & -11.8 & -12.0 & -8.9 & 18.5 & -2.3 & -19.3 \\
\hline 44 & -940.1 & -940. & -940. & -939. & -11.5 & -11.4 & -7.3 & 17.3 & -2.1 & -19.3 \\
\hline 44 & -940.117510 & -940. & 3034 & -939. & -10.7 & -10.4 & -6.9 & 16.3 & -1.7 & -18.1 \\
\hline 44 & -940.117602 & -940.493979 & -940.518141 & -939.020568 & -10.6 & -10.2 & -6.8 & 16.4 & -1.8 & -18.0 \\
\hline 44 & -940.1 & -940. & -940.518403 & -939.0 & -10.4 & -9.8 & -5.8 & 16.5 & -1.7 & -18.8 \\
\hline 44 & -940.1 & -940 & -940.5 & -939. & -10.4 & -10.1 & -6.5 & 15.8 & -1.7 & -17.7 \\
\hline 44 & -940.116633 & -940. & -940.5 & -939. & -10.2 & -9.3 & -5.2 & 15.9 & -1.6 & -18.5 \\
\hline 44 & -940.1 & -940 & -940. & 884 & -10.2 & -10.1 & -6.6 & 15.9 & -1.8 & -17.5 \\
\hline 44 & -940 & -940 & -940. & -939 & -9.7 & -9.7 & -7.9 & 16.3 & -2.1 & -15.9 \\
\hline 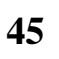 & -1263 & 987 & 350 & 15 & -12.2 & -12.7 & -9.8 & 19.5 & -2.5 & -19.9 \\
\hline 45 & -1263.138046 & -1263. & -1263.502872 & -1261. & -11.7 & -11.6 & -7.7 & 17.4 & -2.2 & -19.1 \\
\hline 45 & -1263.138146 & -1263. & -1263.502668 & -1261. & -11.6 & -11.8 & -7.6 & 17.3 & -2.3 & -19.2 \\
\hline 45 & -1263.137916 & -1263. & -1263. & -1261 & -11.6 & -11.4 & -7.3 & 17.8 & -2.2 & -19.7 \\
\hline 45 & -1263.137893 & -1263.478225 & -1263.502185 & -1261.643196 & -11.4 & -11.5 & -7.3 & 17.8 & -2.3 & -19.9 \\
\hline 45 & -1263.137816 & -1263.478181 & -1263.502347 & -1261.642996 & -11.2 & -11.5 & -7.6 & 16.9 & -2.3 & -18.5 \\
\hline 45 & -1263.137388 & -1263.477319 & -1263.501681 & -1261.642615 & -11.0 & -10.8 & -7.0 & 17.0 & -2.3 & -18.5 \\
\hline 45 & -1263.136324 & -1263.477527 & -1263.502694 & -1261.642601 & -11.0 & -10.2 & -4.9 & 15.5 & -1.5 & -19.3 \\
\hline
\end{tabular}




\begin{tabular}{llllllllllll}
$\mathbf{4 5}$ & -1263.136292 & -1263.477602 & -1263.502952 & -1261.642505 & -10.9 & -10.3 & -5.5 & 16.6 & -1.7 & -19.7 \\
$\mathbf{4 5}$ & -1263.136526 & -1263.477514 & -1263.502392 & -1261.642147 & -10.7 & -10.3 & -5.7 & 15.3 & -1.6 & -18.3 \\
$\mathbf{4 5}$ & -1263.136526 & -1263.477630 & -1263.502948 & -1261.642067 & -10.6 & -10.1 & -5.3 & 15.9 & -1.7 & -19.1 \\
$\mathbf{4 5}$ & -1263.137334 & -1263.476794 & -1263.501252 & -1261.641768 & -10.5 & -10.4 & -7.2 & 15.8 & -2.1 & -17.0 \\
& & & & & & & & & & \\
$\mathbf{4 6}$ & -979.416107 & -979.810867 & -979.833343 & -978.270809 & -13.8 & -14.0 & -12.0 & 22.4 & -3.0 & -21.5 \\
$\mathbf{4 6}$ & -979.414836 & -979.809196 & -979.832267 & -978.269572 & -13.0 & -12.4 & -9.7 & 19.8 & -2.7 & -19.7 \\
$\mathbf{4 6}$ & -979.413617 & -979.808494 & -979.832306 & -978.268985 & -12.7 & -11.8 & -8.4 & 18.7 & -2.4 & -19.8 \\
$\mathbf{4 6}$ & -979.413549 & -979.808259 & -979.831966 & -978.268640 & -12.4 & -12.4 & -9.5 & 19.1 & -2.4 & -19.6 \\
$\mathbf{4 6}$ & -979.413185 & -979.807664 & -979.831149 & -978.268063 & -12.1 & -12.2 & -9.3 & 19.6 & -2.7 & -19.9 \\
$\mathbf{4 6}$ & -979.413279 & -979.807377 & -979.831051 & -978.267838 & -11.9 & -12.0 & -9.3 & 16.8 & -2.5 & -17.0 \\
$\mathbf{4 6}$ & -979.413024 & -979.807476 & -979.831154 & -978.267669 & -11.8 & -11.4 & -9.4 & 20.2 & -2.5 & -19.8 \\
$\mathbf{4 6}$ & -979.412450 & -979.807550 & -979.831773 & -978.267300 & -11.6 & -11.3 & -8.2 & 18.0 & -2.1 & -19.0 \\
$\mathbf{4 6}$ & -979.411273 & -979.806705 & -979.831694 & -978.266830 & -11.3 & -10.7 & -6.6 & 16.8 & -2.0 & -18.9 \\
$\mathbf{4 6}$ & -979.410133 & -979.805430 & -979.830653 & -978.265352 & -10.4 & -9.6 & -6.3 & 17.3 & -1.9 & -18.8 \\
\hline
\end{tabular}


Table S9. Heterocycle Descriptors

\begin{tabular}{|c|c|c|c|c|c|c|c|}
\hline \multirow{2}{*}{ Het. } & \multirow{2}{*}{$\mu(D)$} & \multicolumn{3}{|c|}{ Polarizability } & \multicolumn{3}{|c|}{ Quadrupole Moment } \\
\hline & & $\mathbf{X}^{2}$ & $\mathbf{Y}^{2}$ & $\mathbf{Z}^{2}$ & $\mathbf{X}^{2}$ & $\mathbf{Y}^{2}$ & $\mathbf{Z}^{2}$ \\
\hline 1 & 1.92 & 58.07 & 59.62 & 33.59 & 4.53 & 1.30 & -5.83 \\
\hline 2 & 0.64 & 54.49 & 51.45 & 30.55 & 3.86 & 0.13 & -3.99 \\
\hline 3 & 0.51 & 73.54 & 67.04 & 37.86 & 0.76 & 4.04 & -4.81 \\
\hline 4 & 3.06 & 50.25 & 45.77 & 27.25 & -1.74 & 4.11 & -2.37 \\
\hline 5 & 1.54 & 49.21 & 45.14 & 27.28 & 5.52 & -3.54 & -1.98 \\
\hline 6 & 2.50 & 69.27 & 59.90 & 34.41 & 3.05 & -0.09 & -2.96 \\
\hline 7 & 1.62 & 66.77 & 62.08 & 34.86 & -1.61 & 4.20 & -2.59 \\
\hline 8 & 2.33 & 54.32 & 51.82 & 30.01 & 5.72 & -1.63 & -4.09 \\
\hline 9 & 3.80 & 53.67 & 52.17 & 30.06 & 5.26 & -1.35 & -3.91 \\
\hline 10 & 2.90 & 48.97 & 44.97 & 26.17 & 7.29 & -5.27 & -2.02 \\
\hline 11 & 4.50 & 48.49 & 47.92 & 26.76 & 4.93 & -2.59 & -2.34 \\
\hline 12 & 0.14 & 49.25 & 46.12 & 26.56 & 7.51 & -5.27 & -2.24 \\
\hline 13 & 2.28 & 44.50 & 40.69 & 23.38 & 7.19 & -6.74 & -0.45 \\
\hline 14 & 3.43 & 60.60 & 57.47 & 31.45 & -5.69 & 6.25 & -0.56 \\
\hline 15 & 0.00 & 78.76 & 78.75 & 39.66 & 2.70 & 2.70 & -5.40 \\
\hline 16 & 2.27 & 71.41 & 73.58 & 36.59 & 5.14 & -1.70 & -3.44 \\
\hline 17 & 0.00 & 72.30 & 61.90 & 33.64 & -6.45 & 7.83 & -1.37 \\
\hline 18 & 2.37 & 67.57 & 64.11 & 33.48 & -2.54 & 3.99 & -1.45 \\
\hline 19 & 4.27 & 68.88 & 64.45 & 33.83 & 4.86 & -3.09 & -1.78 \\
\hline 20 & 0.00 & 58.91 & 58.92 & 30.53 & -0.31 & -0.30 & 0.61 \\
\hline 21 & 2.67 & 64.50 & 57.24 & 31.16 & 6.34 & -6.73 & 0.39 \\
\hline 22 & 5.07 & 60.62 & 61.50 & 31.24 & -0.90 & 0.80 & 0.10 \\
\hline 23 & 0.00 & 58.88 & 52.33 & 29.11 & 6.84 & -9.02 & 2.18 \\
\hline 24 & 2.66 & 57.41 & 52.28 & 28.55 & 3.14 & -5.29 & 2.15 \\
\hline 25 & 4.66 & 57.77 & 54.03 & 28.70 & 2.51 & -4.41 & 1.90 \\
\hline 26 & 6.21 & 82.78 & 67.17 & 35.15 & -4.90 & 5.86 & -0.96 \\
\hline 27 & 2.17 & 134.69 & 102.80 & 53.64 & 1.07 & 5.53 & -6.60 \\
\hline 28 & 2.71 & 135.14 & 107.96 & 54.22 & 1.59 & 7.87 & -9.46 \\
\hline 29 & 1.19 & 142.60 & 102.47 & 54.01 & 5.27 & 2.05 & -7.32 \\
\hline 30 & 3.54 & 125.98 & 96.29 & 50.17 & -7.31 & 9.58 & -2.28 \\
\hline 31 & 5.86 & 111.71 & 83.24 & 43.87 & -13.41 & 8.80 & 4.61 \\
\hline 32 & 3.70 & 116.93 & 85.30 & 43.99 & 3.38 & -1.23 & -2.15 \\
\hline 33 & 0.48 & 111.58 & 87.63 & 50.99 & -0.72 & 4.47 & -3.75 \\
\hline 34 & 1.63 & 109.12 & 98.79 & 45.30 & -20.70 & 20.69 & 0.01 \\
\hline 35 & 5.31 & 116.60 & 102.87 & 45.68 & 8.93 & -5.85 & -3.08 \\
\hline 36 & 4.37 & 116.95 & 101.59 & 46.76 & -5.22 & 1.50 & 3.72 \\
\hline 37 & 7.39 & 125.03 & 104.92 & 47.33 & -1.72 & 1.02 & 0.70 \\
\hline 38 & 2.10 & 159.91 & 112.81 & 56.98 & 7.56 & -3.48 & -4.08 \\
\hline 39 & 4.31 & 155.03 & 104.57 & 53.93 & 5.30 & -5.80 & 0.50 \\
\hline 40 & 3.00 & 147.21 & 108.43 & 53.96 & 6.61 & -3.62 & -2.99 \\
\hline 41 & 1.26 & 151.11 & 107.70 & 61.36 & 3.53 & 1.94 & -5.46 \\
\hline
\end{tabular}




\begin{tabular}{cccccccc}
$\mathbf{4 2}$ & 6.15 & 149.00 & 130.21 & 58.73 & 12.86 & -16.17 & 3.30 \\
$\mathbf{4 3}$ & 1.65 & 225.56 & 147.05 & 73.14 & 5.65 & 3.81 & -9.46 \\
$\mathbf{4 4}$ & 0.71 & 215.07 & 139.66 & 70.70 & 4.28 & 5.72 & -10.00 \\
$\mathbf{4 5}$ & 0.81 & 231.15 & 162.37 & 77.54 & 4.43 & 6.64 & -11.07 \\
$\mathbf{4 6}$ & 1.07 & 213.70 & 154.41 & 74.11 & 5.25 & 4.20 & -9.44 \\
\hline $\mathbf{4 7}$ & 1.1 & 46.56 & 39.59 & 23.88 & 7.87 & -6.98 & -0.89 \\
$\mathbf{4 8}$ & 3.1 & 46.51 & 39.42 & 24.04 & 5.84 & -5.18 & -0.66 \\
$\mathbf{4 9}$ & 1.6 & 137.21 & 96.75 & 50.57 & 7.73 & -0.50 & -7.23 \\
$\mathbf{5 0}$ & 3.2 & 137.90 & 98.88 & 50.73 & 4.46 & 1.25 & -5.71 \\
$\mathbf{5 1}$ & 1.7 & 128.08 & 101.90 & 50.47 & -4.44 & 9.87 & -5.43 \\
$\mathbf{5 2}$ & 1.3 & 147.79 & 110.22 & 55.25 & 7.45 & -3.45 & -4.00 \\
$\mathbf{5 3}$ & 0.0 & 171.95 & 123.59 & 59.97 & 3.74 & 3.58 & -7.33 \\
$\mathbf{5 4}$ & 2.4 & 154.20 & 111.71 & 53.97 & 1.23 & 1.44 & -2.67 \\
$\mathbf{5 5}$ & 2.2 & 141.60 & 107.95 & 51.05 & -3.38 & 4.05 & -0.67 \\
$\mathbf{5 6}$ & 4.0 & 183.26 & 120.07 & 58.48 & -9.24 & 11.10 & -1.85 \\
$\mathbf{5 7}$ & 3.7 & 149.25 & 124.63 & 56.46 & 6.93 & -3.62 & -3.31 \\
$\mathbf{5 8}$ & 3.9 & 152.50 & 125.50 & 55.73 & 4.24 & -1.13 & -3.11 \\
\hline Phe & 0.38 & 98.41 & 85.72 & 48.39 & 2.80 & 2.44 & -5.24 \\
Tyr & 1.32 & 109.13 & 88.26 & 50.23 & 1.92 & 3.60 & -5.53 \\
$\mathbf{T r p}$ & 2.04 & 142.78 & 118.74 & 61.80 & 1.39 & 7.47 & -8.87 \\
\hline
\end{tabular}

\begin{tabular}{cccccc}
\hline Het. & $\boldsymbol{E}_{\text {номо }}$ & $\boldsymbol{E}_{\boldsymbol{L U} \boldsymbol{M O}}$ & $\begin{array}{c}\text { Volume } \\
\left(\mathbf{\AA}^{\mathbf{3}}\right)\end{array}$ & $\begin{array}{c}\text { Projected Area } \\
\left(\mathbf{\AA}^{\mathbf{2}}\right)\end{array}$ & $\mathbf{N}_{\mathbf{H A}}$ \\
\hline $\mathbf{1}$ & -182.57 & 63.27 & 68.7 & 29.3 & 5 \\
$\mathbf{2}$ & -195.91 & 45.43 & 65.6 & 27.8 & 5 \\
$\mathbf{3}$ & -200.63 & 29.71 & 74.8 & 30.6 & 5 \\
$\mathbf{4}$ & -223.60 & 26.60 & 61.1 & 26.2 & 5 \\
$\mathbf{5}$ & -212.48 & 35.99 & 60.8 & 25.7 & 5 \\
$\mathbf{6}$ & -215.52 & 15.98 & 69.9 & 28.6 & 5 \\
$\mathbf{7}$ & -211.46 & 19.77 & 70.1 & 28.8 & 5 \\
$\mathbf{8}$ & -208.85 & 49.77 & 63.5 & 27.3 & 5 \\
$\mathbf{9}$ & -196.60 & 55.09 & 63.8 & 27.6 & 5 \\
$\mathbf{1 0}$ & -225.65 & 40.60 & 59.1 & 25.5 & 5 \\
$\mathbf{1 1}$ & -221.11 & 35.60 & 58.9 & 25.3 & 5 \\
$\mathbf{1 2}$ & -227.58 & 33.95 & 58.7 & 25.3 & 5 \\
$\mathbf{1 3}$ & -244.95 & 18.91 & 54.1 & 23.5 & 5 \\
$\mathbf{1 4}$ & -225.97 & 9.71 & 65.1 & 26.6 & 5 \\
$\mathbf{1 5}$ & -208.98 & 34.48 & 83.1 & 34.7 & 6 \\
$\mathbf{1 6}$ & -215.06 & 20.22 & 79 & 33 & 6 \\
$\mathbf{1 7}$ & -212.49 & 3.77 & 73.7 & 30.8 & 6 \\
$\mathbf{1 8}$ & -215.76 & 9.70 & 74.3 & 31.2 & 6 \\
$\mathbf{1 9}$ & -202.42 & 4.12 & 73.9 & 31.1 & 6 \\
$\mathbf{2 0}$ & -230.62 & 2.75 & 68.9 & 28.9 & 6 \\
$\mathbf{2 1}$ & -208.37 & -11.64 & 69.1 & 29.1 & 6 \\
& & & & & 6 \\
\end{tabular}




\begin{tabular}{|c|c|c|c|c|c|}
\hline 22 & -216.32 & -6.68 & 69.3 & 29.1 & 6 \\
\hline 23 & -210.85 & -32.59 & 64.7 & 27.1 & 6 \\
\hline 24 & -220.67 & -24.76 & 64.6 & 27.2 & 6 \\
\hline 25 & -221.45 & -23.47 & 64.9 & 27.2 & 6 \\
\hline 26 & -204.44 & -2.52 & 82.4 & 34.7 & 7 \\
\hline 27 & -176.68 & 29.07 & 112.4 & 46.2 & 9 \\
\hline 28 & -161.72 & 21.89 & 112.8 & 46.4 & 9 \\
\hline 29 & -165.07 & 20.10 & 113.2 & 46.4 & 9 \\
\hline 30 & -192.01 & 23.80 & 107.7 & 44.1 & 9 \\
\hline 31 & -213.77 & 2.84 & 98.4 & 40.5 & 9 \\
\hline 32 & -210.76 & 5.25 & 98.3 & 40.2 & 9 \\
\hline 33 & -179.34 & 34.34 & 107.3 & 42.2 & 9 \\
\hline 34 & -198.71 & 14.36 & 106.3 & 43.8 & 10 \\
\hline 35 & -193.27 & 15.66 & 106.4 & 43.8 & 10 \\
\hline 36 & -199.49 & 8.52 & 114.2 & 47.4 & 11 \\
\hline 37 & -197.30 & 22.90 & 114.6 & 47.4 & 11 \\
\hline 38 & -196.35 & 0.22 & 123 & 49.9 & 10 \\
\hline 39 & -210.88 & -8.43 & 118.4 & 48 & 10 \\
\hline 40 & -206.22 & -8.18 & 118.4 & 47.8 & 10 \\
\hline 41 & -175.25 & 14.70 & 125.7 & 49.6 & 10 \\
\hline 42 & -185.38 & 6.07 & 131.2 & 53.6 & 11 \\
\hline 43 & -175.83 & 14.86 & 156.6 & 62.9 & 13 \\
\hline 44 & -188.41 & 9.24 & 153.5 & 61.3 & 13 \\
\hline 45 & -182.14 & 9.17 & 163.1 & 64.4 & 13 \\
\hline 46 & -180.25 & 24.39 & 169.7 & 66.1 & 14 \\
\hline 47 & -170.24 & -48.08 & 56.4 & 24 & 5 \\
\hline 48 & -157.46 & -39.22 & 56.3 & 24 & 5 \\
\hline 49 & -123.00 & -38.34 & 107.6 & 44.3 & 9 \\
\hline 50 & -120.01 & -42.98 & 108.4 & 44.4 & 9 \\
\hline 51 & -125.88 & -39.76 & 107.9 & 44.4 & 9 \\
\hline 52 & -135.86 & -45.14 & 114.3 & 45.6 & 9 \\
\hline 53 & -123.45 & -44.85 & 127.8 & 51.8 & 10 \\
\hline 54 & -140.22 & -63.69 & 118.4 & 48.2 & 10 \\
\hline 55 & -139.26 & -74.40 & 113.7 & 46 & 10 \\
\hline 56 & -127.65 & -56.61 & 131.1 & 53.4 & 11 \\
\hline 57 & -128.75 & -57.58 & 128.1 & 51.7 & 11 \\
\hline 58 & -123.34 & -58.35 & 126.5 & 51.5 & 11 \\
\hline Phe & -200.60 & 37.14 & 99.8 & 40.3 & 7 \\
\hline Tyr & -184.57 & 35.22 & 107.9 & 43.3 & 8 \\
\hline Trp & -172.40 & 32.45 & 128.3 & 51.0 & 10 \\
\hline
\end{tabular}

\begin{tabular}{llllllll} 
Het. & $E S P_{\max }^{g a s}$ & $E S P_{\min }^{g a s}$ & $E S P_{a v g}^{g a s}$ & $E S P P_{\sigma}^{g a s}$ & $F_{\max }^{g a s}$ & $F_{\text {avg }}^{g a s}$ & $F_{\sigma}^{g a s}$ \\
\hline
\end{tabular} 


\begin{tabular}{|c|c|c|c|c|c|c|c|}
\hline \multirow[b]{2}{*}{1} & \multicolumn{4}{|c|}{ kcal mol ${ }^{-1} \mathrm{e}^{-1}$} & \multicolumn{3}{|c|}{ kcal mol ${ }^{-1} \mathrm{D}^{-1}$} \\
\hline & 2.98 & -11.73 & -5.93 & 3.55 & 1.07 & 0.71 & 0.18 \\
\hline 2 & -0.73 & -6.99 & -3.95 & 1.66 & 0.58 & 0.42 & 0.12 \\
\hline 3 & -0.85 & -7.84 & -4.49 & 1.90 & 0.62 & 0.47 & 0.12 \\
\hline 4 & 3.35 & -8.17 & -1.76 & 3.23 & 0.69 & 0.48 & 0.12 \\
\hline 5 & 2.31 & -6.67 & -1.80 & 2.21 & 0.64 & 0.37 & 0.13 \\
\hline 6 & 2.72 & -7.36 & -2.43 & 2.67 & 0.58 & 0.42 & 0.10 \\
\hline 7 & 1.87 & -6.95 & -2.53 & 2.14 & 0.60 & 0.39 & 0.10 \\
\hline 8 & 3.23 & -9.00 & -3.96 & 3.31 & 0.96 & 0.57 & 0.19 \\
\hline 9 & 6.24 & -11.42 & -3.69 & 4.69 & 1.02 & 0.70 & 0.21 \\
\hline 10 & 6.75 & -7.07 & -1.71 & 3.87 & 0.92 & 0.53 & 0.26 \\
\hline 11 & 6.90 & -10.89 & -1.80 & 5.10 & 1.10 & 0.70 & 0.21 \\
\hline 12 & 2.67 & -4.38 & -2.10 & 1.73 & 0.62 & 0.35 & 0.14 \\
\hline 13 & 6.59 & -6.44 & 0.02 & 3.10 & 0.84 & 0.45 & 0.21 \\
\hline 14 & 4.08 & -8.63 & -0.45 & 3.86 & 0.84 & 0.52 & 0.22 \\
\hline 15 & -1.41 & -8.77 & -4.73 & 2.08 & 0.61 & 0.50 & 0.11 \\
\hline 16 & 2.18 & -7.86 & -2.78 & 2.60 & 0.55 & 0.42 & 0.09 \\
\hline 17 & 1.62 & -3.76 & -0.91 & 1.43 & 0.43 & 0.29 & 0.08 \\
\hline 18 & 4.15 & -4.69 & -0.83 & 2.50 & 0.56 & 0.33 & 0.13 \\
\hline 19 & 5.46 & -9.95 & -0.74 & 4.34 & 0.81 & 0.55 & 0.18 \\
\hline 20 & 2.51 & -1.30 & 1.05 & 0.91 & 0.41 & 0.24 & 0.11 \\
\hline 21 & 6.19 & -6.12 & 1.08 & 3.09 & 0.81 & 0.44 & 0.20 \\
\hline 22 & 8.49 & -9.15 & 1.23 & 5.15 & 0.99 & 0.63 & 0.25 \\
\hline 23 & 5.58 & -1.38 & 2.87 & 1.89 & 0.74 & 0.45 & 0.19 \\
\hline 24 & 6.43 & -4.75 & 2.96 & 3.13 & 0.94 & 0.50 & 0.26 \\
\hline 25 & 10.59 & -6.18 & 3.08 & 5.09 & 1.03 & 0.70 & 0.24 \\
\hline 26 & 9.33 & -13.08 & 0.10 & 6.22 & 1.30 & 0.69 & 0.30 \\
\hline 27 & 3.54 & -11.94 & -6.17 & 3.61 & 0.99 & 0.64 & 0.19 \\
\hline 28 & 4.44 & -13.22 & -6.74 & 4.17 & 1.08 & 0.69 & 0.19 \\
\hline 29 & 1.08 & -10.35 & -5.15 & 2.75 & 0.78 & 0.52 & 0.14 \\
\hline 30 & 6.41 & -10.56 & -4.55 & 4.31 & 1.02 & 0.61 & 0.23 \\
\hline 31 & 11.28 & -10.54 & -0.70 & 5.85 & 1.18 & 0.66 & 0.28 \\
\hline 32 & 8.18 & -6.74 & -1.08 & 3.85 & 0.89 & 0.42 & 0.22 \\
\hline 33 & 4.65 & -7.74 & -3.03 & 2.99 & 0.73 & 0.46 & 0.14 \\
\hline 34 & 6.02 & -9.29 & -0.35 & 3.82 & 0.97 & 0.59 & 0.20 \\
\hline 35 & 7.20 & -13.24 & -1.04 & 5.40 & 1.17 & 0.66 & 0.27 \\
\hline 36 & 8.90 & -7.46 & 1.09 & 3.77 & 0.91 & 0.45 & 0.20 \\
\hline 37 & 14.81 & -12.30 & 0.93 & 6.91 & 1.25 & 0.78 & 0.27 \\
\hline 38 & 1.64 & -8.58 & -3.91 & 2.66 & 0.61 & 0.42 & 0.12 \\
\hline 39 & 4.23 & -11.84 & -2.34 & 4.04 & 0.76 & 0.52 & 0.13 \\
\hline 40 & 2.98 & -6.09 & -1.84 & 2.42 & 0.58 & 0.32 & 0.11 \\
\hline 41 & 3.26 & -9.55 & -4.19 & 3.19 & 0.77 & 0.49 & 0.13 \\
\hline 42 & 8.64 & -14.13 & -3.16 & 5.57 & 0.97 & 0.62 & 0.18 \\
\hline 43 & 3.01 & -10.14 & -5.77 & 2.98 & 0.88 & 0.56 & 0.18 \\
\hline
\end{tabular}




\begin{tabular}{cccccccc}
$\mathbf{4 4}$ & -0.64 & -7.50 & -4.60 & 1.89 & 0.60 & 0.39 & 0.16 \\
$\mathbf{4 5}$ & -0.51 & -8.11 & -4.96 & 2.23 & 0.61 & 0.42 & 0.17 \\
$\mathbf{4 6}$ & 2.54 & -8.05 & -4.37 & 2.46 & 0.80 & 0.46 & 0.17 \\
\hline $\mathbf{4 7}$ & 4.63 & -4.62 & 0.23 & 2.21 & 0.62 & 0.37 & 0.15 \\
$\mathbf{4 8}$ & 4.89 & -7.97 & 0.32 & 4.03 & 0.99 & 0.54 & 0.29 \\
$\mathbf{4 9}$ & 2.05 & -8.91 & -4.83 & 2.78 & 0.78 & 0.49 & 0.17 \\
$\mathbf{5 0}$ & 3.81 & -11.22 & -3.52 & 3.76 & 0.78 & 0.51 & 0.12 \\
$\mathbf{5 1}$ & 2.32 & -7.91 & -4.19 & 2.53 & 0.82 & 0.48 & 0.18 \\
$\mathbf{5 2}$ & 0.77 & -7.07 & -3.67 & 2.12 & 0.62 & 0.38 & 0.13 \\
$\mathbf{5 3}$ & -1.25 & -9.22 & -5.31 & 2.34 & 0.64 & 0.49 & 0.15 \\
$\mathbf{5 4}$ & 3.83 & -6.65 & -2.04 & 2.81 & 0.64 & 0.35 & 0.13 \\
$\mathbf{5 5}$ & 5.22 & -4.48 & 0.00 & 2.36 & 0.57 & 0.32 & 0.12 \\
$\mathbf{5 6}$ & 2.94 & -11.17 & -1.89 & 3.23 & 0.90 & 0.42 & 0.16 \\
$\mathbf{5 7}$ & 3.88 & -10.62 & -2.12 & 3.45 & 0.73 & 0.41 & 0.13 \\
$\mathbf{5 8}$ & 6.40 & -11.95 & -2.00 & 4.17 & 0.80 & 0.47 & 0.18 \\
\hline Phe & 2.02 & -8.88 & -3.74 & 2.90 & 0.87 & 0.49 & 0.16 \\
Tyr & 1.67 & -8.77 & -3.90 & 2.83 & 0.83 & 0.50 & 0.19 \\
Trp & 2.96 & -12.01 & -5.60 & 3.57 & 0.97 & 0.61 & 0.19 \\
\hline
\end{tabular}

Table S10. Cross Validation of Equation (1) from main text

$$
\Delta E_{\text {pred }}=N_{H A}^{A A}\left(-0.036 E S P_{\text {mean }}^{\text {Het }}-0.013 E S P_{\text {range }}^{\text {Het }}-0.095 N_{H A}^{H e t}\right)-1.36
$$

\begin{tabular}{cccccccc}
\hline Amino Acid & Het. & Comp. & Pred. & Leave 1 Out & 10-fold & 5-fold & 3-fold \\
\hline Phe & $\mathbf{2}$ & -4.2 & -4.3 & -4.2 & -4.2 & -4.1 & -4.1 \\
Phe & $\mathbf{3}$ & -4.5 & -4.2 & -4.1 & -4.1 & -4.1 & -4.1 \\
Phe & $\mathbf{4}$ & -5.1 & -5.3 & -5.2 & -5.2 & -5.2 & -5.2 \\
Phe & $\mathbf{5}$ & -5.1 & -5.0 & -5.0 & -5.0 & -4.9 & -5.0 \\
Phe & $\mathbf{6}$ & -5.5 & -5.0 & -4.9 & -4.9 & -4.9 & -4.9 \\
Phe & $\mathbf{7}$ & -5.5 & -4.8 & -4.8 & -4.8 & -4.7 & -4.8 \\
Phe & $\mathbf{8}$ & -3.6 & -4.8 & -4.7 & -4.7 & -4.7 & -4.7 \\
Phe & $\mathbf{1 0}$ & -4.3 & -5.5 & -5.5 & -5.4 & -5.4 & -5.5 \\
Phe & $\mathbf{1 2}$ & -4.8 & -4.8 & -4.7 & -4.7 & -4.7 & -4.7 \\
Phe & $\mathbf{1 4}$ & -5.2 & -5.9 & -5.8 & -5.8 & -5.8 & -5.8 \\
Phe & $\mathbf{1 5}$ & -6.1 & -5.7 & -5.6 & -5.7 & -5.6 & -5.7 \\
Phe & $\mathbf{1 6}$ & -4.5 & -4.8 & -4.8 & -4.8 & -4.7 & -4.7 \\
Phe & $\mathbf{1 7}$ & -5.6 & -5.6 & -5.5 & -5.5 & -5.5 & -5.5 \\
Phe & $\mathbf{1 8}$ & -6.0 & -5.6 & -5.5 & -5.5 & -5.5 & -5.5 \\
Phe & $\mathbf{1 9}$ & -6.1 & -5.9 & -5.9 & -5.8 & -5.8 & -5.9 \\
Phe & $\mathbf{2 0}$ & -6.5 & -6.6 & -6.5 & -6.5 & -6.5 & -6.5 \\
Phe & $\mathbf{2 1}$ & -5.9 & -6.0 & -5.9 & -5.9 & -5.8 & -5.9 \\
Phe & $\mathbf{2 2}$ & -7.0 & -6.7 & -6.7 & -6.6 & -6.6 & -6.7 \\
Phe & $\mathbf{2 3}$ & -7.3 & -7.3 & -7.2 & -7.2 & -7.2 & -7.3
\end{tabular}




\begin{tabular}{|c|c|c|c|c|c|c|c|}
\hline Phe & 24 & -6.8 & -6.7 & -6.7 & -6.6 & -6.6 & -6.7 \\
\hline Phe & 25 & -7.1 & -7.1 & -7.1 & -7.0 & -7.0 & -7.1 \\
\hline Phe & 26 & -8.0 & -7.7 & -7.6 & -7.6 & -7.6 & -7.7 \\
\hline Phe & 27 & -7.3 & -8.1 & -8.0 & -8.0 & -8.0 & -8.1 \\
\hline Phe & 28 & -7.3 & -7.2 & -7.1 & -7.1 & -7.1 & -7.2 \\
\hline Phe & 29 & -7.1 & -7.3 & -7.2 & -7.2 & -7.2 & -7.2 \\
\hline Phe & 30 & -7.6 & -7.1 & -7.0 & -7.0 & -7.0 & -7.0 \\
\hline Phe & 31 & -6.4 & -7.7 & -7.7 & -7.7 & -7.8 & -7.7 \\
\hline Phe & 32 & -9.4 & -9.2 & -9.1 & -9.2 & -9.2 & -9.2 \\
\hline Phe & 33 & -7.9 & -8.4 & -8.4 & -8.4 & -8.5 & -8.4 \\
\hline Phe & 34 & -7.6 & -7.7 & -7.6 & -7.7 & -7.7 & -7.7 \\
\hline Phe & 35 & -9.4 & -9.3 & -9.3 & -9.3 & -9.4 & -9.3 \\
\hline Phe & 36 & -9.2 & -9.6 & -9.5 & -9.6 & -9.7 & -9.7 \\
\hline Phe & 37 & -9.4 & -10.4 & -10.4 & -10.5 & -10.5 & -10.5 \\
\hline Phe & 38 & -8.8 & -11.4 & -11.4 & -11.5 & -11.5 & -11.5 \\
\hline Phe & 39 & -8.1 & -8.0 & -7.9 & -7.9 & -7.9 & -7.9 \\
\hline Phe & 40 & -8.9 & -8.9 & -8.8 & -8.9 & -8.9 & -8.9 \\
\hline Phe & 41 & -8.6 & -8.4 & -8.3 & -8.3 & -8.4 & -8.3 \\
\hline Phe & 42 & -7.7 & -8.1 & -8.1 & -8.1 & -8.1 & -8.1 \\
\hline Phe & 43 & -9.1 & -10.0 & -9.9 & -10.0 & -10.0 & -10.0 \\
\hline Phe & 44 & -10.0 & -9.7 & -9.7 & -9.7 & -9.7 & -9.7 \\
\hline Phe & 45 & -8.8 & -9.5 & -9.5 & -9.5 & -9.5 & -9.4 \\
\hline Phe & 46 & -9.2 & -9.4 & -9.4 & -9.4 & -9.4 & -9.4 \\
\hline Phe & 47 & -9.8 & -10.5 & -10.5 & -10.5 & -10.5 & -10.5 \\
\hline Tyr & 2 & -4.3 & -4.7 & -4.6 & -4.7 & -4.6 & -4.6 \\
\hline Tyr & 3 & -5.2 & -4.6 & -4.5 & -4.6 & -4.6 & -4.6 \\
\hline Tyr & 4 & -5.1 & -5.9 & -5.8 & -5.8 & -5.9 & -5.9 \\
\hline Tyr & 5 & -5.1 & -5.6 & -5.5 & -5.6 & -5.6 & -5.6 \\
\hline Tyr & 6 & -5.8 & -5.5 & -5.4 & -5.5 & -5.5 & -5.6 \\
\hline Tyr & 7 & -5.5 & -5.3 & -5.3 & -5.3 & -5.3 & -5.4 \\
\hline Tyr & 8 & -4.5 & -5.3 & -5.2 & -5.3 & -5.3 & -5.4 \\
\hline Tyr & 10 & -4.3 & -6.1 & -6.1 & -6.1 & -6.1 & -6.2 \\
\hline Tyr & 12 & -5.1 & -5.3 & -5.2 & -5.3 & -5.3 & -5.3 \\
\hline Tyr & 14 & -4.8 & -6.5 & -6.5 & -6.5 & -6.5 & -6.6 \\
\hline Tyr & 15 & -6.3 & -6.4 & -6.3 & -6.3 & -6.3 & -6.4 \\
\hline Tyr & 16 & -4.9 & -5.3 & -5.3 & -5.3 & -5.2 & -5.3 \\
\hline Tyr & 17 & -5.8 & -6.2 & -6.1 & -6.1 & -6.1 & -6.2 \\
\hline Tyr & 18 & -6.4 & -6.2 & -6.2 & -6.2 & -6.2 & -6.2 \\
\hline Tyr & 19 & -6.4 & -6.6 & -6.5 & -6.6 & -6.6 & -6.6 \\
\hline Tyr & 20 & -6.7 & -7.3 & -7.2 & -7.3 & -7.3 & -7.4 \\
\hline Tyr & 21 & -6.6 & -6.6 & -6.6 & -6.6 & -6.6 & -6.6 \\
\hline Tyr & 22 & -7.6 & -7.5 & -7.4 & -7.5 & -7.5 & -7.6 \\
\hline Tyr & 23 & -8.6 & -8.1 & -8.0 & -8.1 & -8.1 & -8.2 \\
\hline Tyr & 24 & -7.2 & -7.5 & -7.4 & -7.5 & -7.5 & -7.4 \\
\hline
\end{tabular}




\begin{tabular}{|c|c|c|c|c|c|c|c|}
\hline Tyr & 25 & -7.8 & -7.9 & -7.9 & -7.9 & -8.0 & -8.0 \\
\hline Tyr & 26 & -8.7 & -8.6 & -8.5 & -8.5 & -8.6 & -8.6 \\
\hline Tyr & 27 & -7.3 & -9.0 & -9.0 & -9.0 & -9.1 & -9.2 \\
\hline Tyr & 28 & -8.6 & -8.0 & -7.9 & -7.9 & -7.9 & -8.1 \\
\hline Tyr & 29 & -7.8 & -8.1 & -8.0 & -8.0 & -8.0 & -8.2 \\
\hline Tyr & 30 & -8.1 & -7.9 & -7.8 & -7.8 & -7.8 & -7.9 \\
\hline Tyr & 31 & -9.3 & -8.7 & -8.6 & -8.6 & -8.6 & -8.7 \\
\hline Tyr & 32 & -11.2 & -10.3 & -10.2 & -10.3 & -10.3 & -10.4 \\
\hline Tyr & 33 & -9.1 & -9.4 & -9.4 & -9.4 & -9.4 & -9.5 \\
\hline Tyr & 34 & -8.3 & -8.6 & -8.6 & -8.5 & -8.6 & -8.6 \\
\hline Tyr & 35 & -11.7 & -10.5 & -10.4 & -10.4 & -10.5 & -10.5 \\
\hline Tyr & 36 & -10.5 & -10.8 & -10.7 & -10.8 & -10.8 & -10.9 \\
\hline Tyr & 37 & -11.5 & -11.7 & -11.7 & -11.7 & -11.8 & -11.8 \\
\hline Tyr & 38 & -11.2 & -12.8 & -12.8 & -12.9 & -12.9 & -12.9 \\
\hline Tyr & 39 & -8.5 & -8.9 & -8.8 & -8.8 & -8.8 & -8.9 \\
\hline Tyr & 40 & -9.2 & -10.0 & -9.9 & -9.9 & -9.9 & -10.0 \\
\hline Tyr & 41 & -9.3 & -9.4 & -9.3 & -9.4 & -9.3 & -9.3 \\
\hline Tyr & 42 & -9.1 & -9.1 & -9.0 & -9.1 & -9.0 & -9.1 \\
\hline Tyr & 43 & -10.1 & -11.2 & -11.1 & -11.2 & -11.2 & -11.3 \\
\hline Tyr & 44 & -11.2 & -10.9 & -10.9 & -11.0 & -11.0 & -10.9 \\
\hline Tyr & 45 & -9.9 & -10.6 & -10.6 & -10.7 & -10.6 & -10.5 \\
\hline Tyr & 46 & -10.2 & -10.6 & -10.6 & -10.6 & -10.6 & -10.5 \\
\hline Tyr & 47 & -11.4 & -11.8 & -11.8 & -11.9 & -11.8 & -11.8 \\
\hline Trp & 1 & -4.5 & -5.9 & -5.9 & -5.9 & -5.9 & -6.0 \\
\hline Trp & 2 & -5.3 & -5.5 & -5.4 & -5.5 & -5.4 & -5.4 \\
\hline Trp & 3 & -5.8 & -5.4 & -5.3 & -5.4 & -5.3 & -5.3 \\
\hline Trp & 4 & -7.2 & -7.0 & -6.9 & -6.9 & -6.9 & -6.6 \\
\hline Trp & 5 & -6.7 & -6.6 & -6.6 & -6.6 & -6.5 & -6.4 \\
\hline Trp & 6 & -7.2 & -6.5 & -6.5 & -6.4 & -6.5 & -6.3 \\
\hline Trp & 7 & -7.3 & -6.3 & -6.3 & -6.2 & -6.3 & -6.1 \\
\hline Trp & 8 & -5.1 & -6.3 & -6.2 & -6.1 & -6.2 & -5.8 \\
\hline $\operatorname{Trp}$ & 9 & -8.4 & -7.1 & -6.9 & -6.9 & -7.0 & -6.3 \\
\hline Trp & 11 & -6.0 & -7.8 & -7.8 & -7.6 & -7.7 & -7.0 \\
\hline Trp & 12 & -6.2 & -6.3 & -6.2 & -6.1 & -6.2 & -6.2 \\
\hline Trp & 15 & -9.1 & -7.6 & -7.5 & -7.5 & -7.5 & -7.2 \\
\hline Trp & 16 & -6.0 & -6.3 & -6.2 & -6.2 & -6.3 & -6.2 \\
\hline Trp & 17 & -7.7 & -7.4 & -7.3 & -7.2 & -7.3 & -7.1 \\
\hline $\operatorname{Trp}$ & 18 & -8.1 & -7.4 & -7.4 & -7.3 & -7.3 & -7.4 \\
\hline Trp & 19 & -8.4 & -7.9 & -7.8 & -7.8 & -7.8 & -7.7 \\
\hline Trp & 20 & -9.5 & -8.8 & -8.7 & -8.7 & -8.7 & -8.2 \\
\hline Trp & 21 & -8.4 & -7.9 & -7.9 & -7.8 & -7.8 & -8.1 \\
\hline $\operatorname{Trp}$ & 22 & -9.9 & -9.0 & -9.0 & -8.9 & -8.8 & -8.7 \\
\hline Trp & 23 & -10.3 & -9.8 & -9.7 & -9.5 & -9.3 & -9.1 \\
\hline $\operatorname{Trp}$ & 24 & -9.8 & -9.0 & -8.9 & -9.0 & -9.0 & -9.0 \\
\hline
\end{tabular}




\begin{tabular}{llllllll} 
Trp & $\mathbf{2 5}$ & -9.9 & -9.6 & -9.5 & -9.5 & -9.4 & -9.3 \\
Trp & $\mathbf{2 6}$ & -10.8 & -10.3 & -10.3 & -10.2 & -9.9 & -9.8 \\
Trp & $\mathbf{2 7}$ & -10.4 & -11.0 & -10.9 & -10.6 & -10.2 & -10.0 \\
Trp & $\mathbf{2 8}$ & -11.2 & -9.7 & -9.5 & -9.3 & -9.2 & -9.1 \\
Trp & $\mathbf{2 9}$ & -10.4 & -9.8 & -9.6 & -9.4 & -9.2 & -9.0 \\
Trp & $\mathbf{3 0}$ & -11.0 & -9.5 & -9.4 & -9.3 & -9.2 & -9.2 \\
Trp & $\mathbf{3 1}$ & -11.8 & -10.5 & -10.3 & -10.1 & -9.9 & -9.8 \\
Trp & $\mathbf{3 2}$ & -13.6 & -12.5 & -12.4 & -12.1 & -11.7 & -11.6 \\
Trp & $\mathbf{3 3}$ & -12.4 & -11.5 & -11.4 & -11.2 & -11.0 & -10.9 \\
Trp & $\mathbf{3 4}$ & -10.2 & -10.4 & -10.4 & -10.2 & -10.1 & -10.0 \\
Trp & $\mathbf{3 5}$ & -14.0 & -12.7 & -12.6 & -12.7 & -12.2 & -12.2 \\
Trp & $\mathbf{3 6}$ & -14.4 & -13.1 & -13.0 & -13.0 & -12.4 & -12.3 \\
Trp & $\mathbf{3 7}$ & -14.2 & -14.3 & -14.3 & -14.3 & -13.8 & -13.8 \\
Trp & $\mathbf{3 8}$ & -15.8 & -15.7 & -15.6 & -15.5 & -14.6 & -14.5 \\
Trp & $\mathbf{3 9}$ & -10.5 & -10.8 & -10.7 & -10.8 & -10.5 & -10.5 \\
Trp & $\mathbf{4 0}$ & -11.8 & -12.1 & -12.1 & -12.1 & -11.6 & -11.5 \\
Trp & $\mathbf{4 1}$ & -10.7 & -11.4 & -11.4 & -11.5 & -11.2 & -11.2 \\
Trp & $\mathbf{4 2}$ & -10.9 & -11.0 & -11.0 & -11.0 & -10.6 & -10.6 \\
Trp & $\mathbf{4 3}$ & -13.3 & -13.6 & -13.6 & -13.5 & -12.8 & -12.6 \\
Trp & $\mathbf{4 4}$ & -14.2 & -13.3 & -13.2 & -13.5 & -12.9 & -12.9 \\
Trp & $\mathbf{4 5}$ & -11.8 & -12.9 & -13.0 & -13.2 & -12.8 & -12.9 \\
Trp & $\mathbf{4 6}$ & -12.2 & -12.9 & -12.9 & -13.1 & -12.7 & -12.8 \\
Trp & $\mathbf{4 7}$ & -13.8 & -14.5 & -14.5 & -14.7 & -14.1 & -14.2 \\
\hline $\mathbf{R} \mathbf{2}$ & & -- & 0.92 & 0.92 & 0.91 & 0.90 & 0.89 \\
$\mathbf{M A E}$ & & -- & 0.56 & 0.58 & 0.59 & 0.62 & 0.65 \\
$\mathbf{R M S E}$ & & -- & 0.74 & 0.76 & 0.78 & 0.83 & 0.87 \\
\hline
\end{tabular}

Table S11. Cross Validation of Equation (4)

$$
\Delta E_{\text {pred }}=N_{H A}^{A A}\left(-0.032 E S P_{\max }^{H e t}-0.087 N_{H A}^{H e t}\right)-1.46
$$

\begin{tabular}{cccccccc}
\hline Amino Acid & Het. & Comp. & Pred. & Leave 1 Out & 10-fold & 5-fold & 3-fold \\
\hline Phe & $\mathbf{2}$ & -4.2 & -4.3 & -4.3 & -4.3 & -4.2 & -4.2 \\
Phe & $\mathbf{3}$ & -4.5 & -4.3 & -4.2 & -4.3 & -4.2 & -4.2 \\
Phe & $\mathbf{4}$ & -5.1 & -5.3 & -5.2 & -5.2 & -5.1 & -5.2 \\
Phe & $\mathbf{5}$ & -5.1 & -5.0 & -4.9 & -5.0 & -4.9 & -4.9 \\
Phe & $\mathbf{6}$ & -5.5 & -5.1 & -5.0 & -5.1 & -5.0 & -5.0 \\
Phe & $\mathbf{7}$ & -5.5 & -4.9 & -4.8 & -4.9 & -4.8 & -4.8 \\
Phe & $\mathbf{8}$ & -3.6 & -5.2 & -5.2 & -5.2 & -5.1 & -5.1 \\
Phe & $\mathbf{1 0}$ & -4.3 & -6.0 & -6.0 & -6.0 & -5.9 & -6.0 \\
Phe & $\mathbf{1 2}$ & -4.8 & -5.1 & -5.0 & -5.1 & -5.0 & -5.0 \\
Phe & $\mathbf{1 4}$ & -5.2 & -6.0 & -5.9 & -5.9 & -5.9 & -5.9 \\
Phe & $\mathbf{1 5}$ & -6.1 & -5.4 & -5.3 & -5.4 & -5.3 & -5.3 \\
Phe & $\mathbf{1 6}$ & -4.5 & -4.8 & -4.7 & -4.7 & -4.7 & -4.6
\end{tabular}




\begin{tabular}{|c|c|c|c|c|c|c|c|}
\hline Phe & 17 & -5.6 & -5.6 & -5.5 & -5.6 & -5.5 & -5.5 \\
\hline Phe & 18 & -6.0 & -5.5 & -5.4 & -5.3 & -5.4 & -5.4 \\
\hline Phe & 19 & -6.1 & -6.0 & -6.0 & -5.9 & -5.9 & -6.0 \\
\hline Phe & 20 & -6.5 & -6.3 & -6.2 & -6.2 & -6.2 & -6.3 \\
\hline Phe & 21 & -5.9 & -5.7 & -5.6 & -5.5 & -5.6 & -5.6 \\
\hline Phe & 22 & -7.0 & -6.5 & -6.4 & -6.4 & -6.4 & -6.5 \\
\hline Phe & 23 & -7.3 & -7.0 & -6.9 & -6.9 & -6.9 & -7.0 \\
\hline Phe & 24 & -6.8 & -6.4 & -6.3 & -6.2 & -6.2 & -6.3 \\
\hline Phe & 25 & -7.1 & -6.6 & -6.5 & -6.4 & -6.4 & -6.5 \\
\hline Phe & 26 & -8.0 & -7.5 & -7.4 & -7.3 & -7.4 & -7.5 \\
\hline Phe & 27 & -7.3 & -7.8 & -7.7 & -7.7 & -7.7 & -7.8 \\
\hline Phe & 28 & -7.3 & -7.7 & -7.7 & -7.6 & -7.6 & -7.7 \\
\hline Phe & 29 & -7.1 & -7.9 & -7.9 & -7.8 & -7.8 & -7.9 \\
\hline Phe & 30 & -7.6 & -7.2 & -7.1 & -7.1 & -7.1 & -7.1 \\
\hline Phe & 31 & -6.4 & -8.4 & -8.3 & -8.4 & -8.4 & -8.4 \\
\hline Phe & 32 & -9.4 & -9.5 & -9.4 & -9.5 & -9.6 & -9.5 \\
\hline Phe & 33 & -7.9 & -8.8 & -8.7 & -8.8 & -8.9 & -8.8 \\
\hline Phe & 34 & -7.6 & -8.0 & -7.9 & -8.0 & -8.0 & -8.0 \\
\hline Phe & 35 & -9.4 & -8.9 & -8.8 & -8.9 & -8.9 & -8.9 \\
\hline Phe & 36 & -9.2 & -9.2 & -9.1 & -9.2 & -9.2 & -9.2 \\
\hline Phe & 37 & -9.4 & -10.2 & -10.1 & -10.2 & -10.2 & -10.2 \\
\hline Phe & 38 & -8.8 & -11.5 & -11.5 & -11.6 & -11.7 & -11.6 \\
\hline Phe & 39 & -8.1 & -7.9 & -7.8 & -7.8 & -7.9 & -7.9 \\
\hline Phe & 40 & -8.9 & -8.5 & -8.4 & -8.5 & -8.5 & -8.5 \\
\hline Phe & 41 & -8.6 & -8.2 & -8.1 & -8.2 & -8.2 & -8.2 \\
\hline Phe & 42 & -7.7 & -8.3 & -8.2 & -8.2 & -8.3 & -8.2 \\
\hline Phe & 43 & -9.1 & -10.1 & -10.0 & -10.1 & -10.2 & -10.2 \\
\hline Phe & 44 & -10.0 & -10.1 & -10.0 & -10.0 & -10.0 & -10.0 \\
\hline Phe & 45 & -8.8 & -9.2 & -9.2 & -9.2 & -9.2 & -9.2 \\
\hline Phe & 46 & -9.2 & -9.3 & -9.2 & -9.2 & -9.2 & -9.2 \\
\hline Phe & 47 & -9.8 & -10.6 & -10.5 & -10.5 & -10.5 & -10.6 \\
\hline Tyr & 2 & -4.3 & -4.8 & -4.7 & -4.7 & -4.7 & -4.6 \\
\hline Tyr & 3 & -5.2 & -4.7 & -4.6 & -4.7 & -4.7 & -4.7 \\
\hline Tyr & 4 & -5.1 & -5.8 & -5.7 & -5.8 & -5.8 & -5.8 \\
\hline Tyr & 5 & -5.1 & -5.5 & -5.4 & -5.5 & -5.5 & -5.6 \\
\hline Tyr & 6 & -5.8 & -5.6 & -5.5 & -5.6 & -5.6 & -5.7 \\
\hline Tyr & 7 & -5.5 & -5.4 & -5.3 & -5.4 & -5.4 & -5.4 \\
\hline Tyr & 8 & -4.5 & -5.8 & -5.7 & -5.8 & -5.8 & -5.8 \\
\hline Tyr & 10 & -4.3 & -6.7 & -6.6 & -6.6 & -6.7 & -6.7 \\
\hline Tyr & 12 & -5.1 & -5.6 & -5.5 & -5.6 & -5.6 & -5.7 \\
\hline Tyr & 14 & -4.8 & -6.6 & -6.6 & -6.5 & -6.6 & -6.7 \\
\hline Tyr & 15 & -6.3 & -6.0 & -5.9 & -5.9 & -5.9 & -6.0 \\
\hline Tyr & 16 & -4.9 & -5.3 & -5.2 & -5.2 & -5.2 & -5.3 \\
\hline Tyr & 17 & -5.8 & -6.2 & -6.1 & -6.1 & -6.1 & -6.2 \\
\hline
\end{tabular}




\begin{tabular}{|c|c|c|c|c|c|c|c|}
\hline Tyr & 18 & -6.4 & -6.1 & -6.0 & -6.0 & -6.0 & -6.1 \\
\hline Tyr & 19 & -6.4 & -6.7 & -6.6 & -6.6 & -6.6 & -6.7 \\
\hline Tyr & 20 & -6.7 & -7.0 & -7.0 & -6.9 & -7.0 & -7.1 \\
\hline Tyr & 21 & -6.6 & -6.3 & -6.2 & -6.2 & -6.2 & -6.3 \\
\hline Tyr & 22 & -7.6 & -7.2 & -7.1 & -7.1 & -7.2 & -7.3 \\
\hline Tyr & 23 & -8.6 & -7.8 & -7.7 & -7.7 & -7.8 & -7.9 \\
\hline Tyr & 24 & -7.2 & -7.1 & -7.0 & -7.0 & -7.0 & -7.1 \\
\hline Tyr & 25 & -7.8 & -7.3 & -7.2 & -7.2 & -7.2 & -7.4 \\
\hline Tyr & 26 & -8.7 & -8.3 & -8.2 & -8.2 & -8.3 & -8.5 \\
\hline Tyr & 27 & -7.3 & -8.7 & -8.7 & -8.7 & -8.7 & -8.8 \\
\hline Tyr & 28 & -8.6 & -8.6 & -8.6 & -8.6 & -8.6 & -8.7 \\
\hline Tyr & 29 & -7.8 & -8.9 & -8.8 & -8.8 & -8.8 & -8.9 \\
\hline Tyr & 30 & -8.1 & -8.0 & -7.9 & -7.9 & -7.9 & -8.0 \\
\hline Tyr & 31 & -9.3 & -9.4 & -9.3 & -9.3 & -9.3 & -9.4 \\
\hline Tyr & 32 & -11.2 & -10.6 & -10.5 & -10.6 & -10.6 & -10.7 \\
\hline Tyr & 33 & -9.1 & -9.8 & -9.8 & -9.8 & -9.8 & -9.9 \\
\hline Tyr & 34 & -8.3 & -8.9 & -8.9 & -8.9 & -8.9 & -9.0 \\
\hline Tyr & 35 & -11.7 & -10.0 & -9.9 & -9.9 & -9.9 & -10.0 \\
\hline Tyr & 36 & -10.5 & -10.3 & -10.2 & -10.3 & -10.2 & -10.3 \\
\hline Tyr & 37 & -11.5 & -11.4 & -11.3 & -11.4 & -11.4 & -11.5 \\
\hline Tyr & 38 & -11.2 & -12.9 & -13.0 & -13.0 & -12.9 & -13.1 \\
\hline Tyr & 39 & -8.5 & -8.8 & -8.8 & -8.8 & -8.8 & -8.8 \\
\hline Tyr & 40 & -9.2 & -9.5 & -9.4 & -9.5 & -9.5 & -9.5 \\
\hline Tyr & 41 & -9.3 & -9.2 & -9.1 & -9.2 & -9.1 & -9.2 \\
\hline Tyr & 42 & -9.1 & -9.3 & -9.2 & -9.2 & -9.2 & -9.3 \\
\hline Tyr & 43 & -10.1 & -11.3 & -11.3 & -11.3 & -11.3 & -11.4 \\
\hline Tyr & 44 & -11.2 & -11.3 & -11.2 & -11.3 & -11.3 & -11.3 \\
\hline Tyr & 45 & -9.9 & -10.3 & -10.3 & -10.4 & -10.3 & -10.3 \\
\hline Tyr & 46 & -10.2 & -10.4 & -10.4 & -10.4 & -10.4 & -10.3 \\
\hline Tyr & 47 & -11.4 & -11.9 & -11.8 & -11.9 & -11.9 & -11.8 \\
\hline Trp & 1 & -4.5 & -6.8 & -6.7 & -6.7 & -6.7 & -6.8 \\
\hline $\operatorname{Trp}$ & 2 & -5.3 & -5.6 & -5.5 & -5.5 & -5.5 & -5.6 \\
\hline $\operatorname{Trp}$ & 3 & -5.8 & -5.5 & -5.5 & -5.5 & -5.4 & -5.6 \\
\hline Trp & 4 & -7.2 & -6.9 & -6.8 & -6.8 & -6.8 & -6.6 \\
\hline Trp & 5 & -6.7 & -6.5 & -6.5 & -6.5 & -6.4 & -6.4 \\
\hline Trp & 6 & -7.2 & -6.7 & -6.6 & -6.6 & -6.6 & -6.5 \\
\hline Trp & 7 & -7.3 & -6.4 & -6.3 & -6.3 & -6.3 & -6.3 \\
\hline $\operatorname{Trp}$ & 8 & -5.1 & -6.8 & -6.8 & -6.7 & -6.7 & -6.6 \\
\hline Trp & 9 & -8.4 & -7.8 & -7.7 & -7.7 & -7.7 & -7.4 \\
\hline Trp & 11 & -6.0 & -8.0 & -8.0 & -7.9 & -7.9 & -7.5 \\
\hline Trp & 12 & -6.2 & -6.7 & -6.6 & -6.5 & -6.6 & -6.5 \\
\hline $\operatorname{Trp}$ & 15 & -9.1 & -7.1 & -7.0 & -7.0 & -7.0 & -6.8 \\
\hline Trp & 16 & -6.0 & -6.2 & -6.2 & -6.1 & -6.1 & -6.3 \\
\hline $\operatorname{Trp}$ & 17 & -7.7 & -7.4 & -7.3 & -7.3 & -7.3 & -7.2 \\
\hline
\end{tabular}




\begin{tabular}{|c|c|c|c|c|c|c|c|}
\hline Trp & 18 & -8.1 & -7.2 & -7.1 & -7.1 & -7.1 & -7.1 \\
\hline Trp & 19 & -8.4 & -8.0 & -7.9 & -7.9 & -7.9 & -7.7 \\
\hline Trp & 20 & -9.5 & -8.4 & -8.3 & -8.3 & -8.3 & -8.0 \\
\hline Trp & 21 & -8.4 & -7.5 & -7.4 & -7.4 & -7.4 & -7.3 \\
\hline Trp & 22 & -9.9 & -8.7 & -8.6 & -8.4 & -8.3 & -8.2 \\
\hline Trp & 23 & -10.3 & -9.4 & -9.3 & -9.1 & -8.9 & -8.8 \\
\hline Trp & 24 & -9.8 & -8.5 & -8.4 & -8.3 & -8.1 & -8.1 \\
\hline Trp & 25 & -9.9 & -8.7 & -8.6 & -8.5 & -8.3 & -8.3 \\
\hline Trp & 26 & -10.8 & -10.1 & -9.9 & -9.7 & -9.4 & -9.3 \\
\hline Trp & 27 & -10.4 & -10.5 & -10.5 & -10.2 & -9.9 & -9.9 \\
\hline Trp & 28 & -11.2 & -10.4 & -10.4 & -10.3 & -10.1 & -10.1 \\
\hline Trp & 29 & -10.4 & -10.7 & -10.7 & -10.5 & -10.4 & -10.3 \\
\hline Trp & 30 & -11.0 & -9.6 & -9.6 & -9.6 & -9.5 & -9.5 \\
\hline Trp & 31 & -11.8 & -11.3 & -11.3 & -11.1 & -10.9 & -10.8 \\
\hline Trp & 32 & -13.6 & -12.9 & -12.8 & -12.5 & -12.1 & -12.0 \\
\hline Trp & 33 & -12.4 & -11.9 & -11.8 & -11.6 & -11.3 & -11.3 \\
\hline Trp & 34 & -10.2 & -10.8 & -10.7 & -10.6 & -10.4 & -10.4 \\
\hline Trp & 35 & -14.0 & -12.1 & -12.0 & -12.0 & -11.6 & -11.6 \\
\hline Trp & 36 & -14.4 & -12.5 & -12.4 & -12.3 & -11.9 & -11.9 \\
\hline Trp & 37 & -14.2 & -13.9 & -13.8 & -13.7 & -13.2 & -13.1 \\
\hline Trp & 38 & -15.8 & -15.8 & -15.7 & -15.5 & -14.7 & -14.6 \\
\hline Trp & 39 & -10.5 & -10.7 & -10.6 & -10.6 & -10.5 & -10.4 \\
\hline Trp & 40 & -11.8 & -11.5 & -11.5 & -11.4 & -11.2 & -11.1 \\
\hline Trp & 41 & -10.7 & -11.1 & -11.1 & -11.0 & -10.8 & -10.8 \\
\hline Trp & 42 & -10.9 & -11.2 & -11.2 & -11.1 & -10.9 & -10.9 \\
\hline Trp & 43 & -13.3 & -13.8 & -13.8 & -13.6 & -13.1 & -13.1 \\
\hline Trp & 44 & -14.2 & -13.7 & -13.7 & -13.7 & -13.4 & -13.3 \\
\hline Trp & 45 & -11.8 & -12.6 & -12.6 & -12.6 & -12.5 & -12.4 \\
\hline Trp & 46 & -12.2 & -12.6 & -12.6 & -12.6 & -12.5 & -12.4 \\
\hline Trp & 47 & -13.8 & -14.5 & -14.5 & -14.4 & -14.1 & -14.1 \\
\hline $\mathbf{R}^{2}$ & & -- & 0.90 & 0.89 & 0.89 & 0.88 & 0.87 \\
\hline MAE & & -- & 0.63 & 0.65 & 0.67 & 0.69 & 0.71 \\
\hline RMSE & & -- & 0.84 & 0.86 & 0.88 & 0.93 & 0.95 \\
\hline
\end{tabular}

Table S12. Absolute energies and binding energies for all unique dimers of 47-58 with the model toluene (Phe).

\begin{tabular}{cccc}
\hline & $\begin{array}{c}\text { B97D } \\
\text { Het. }\end{array}$ & \multicolumn{2}{c}{$\begin{array}{c}\text { DLPNO-CCSD(T) } \\
\text { /def-TZVPPQ }\end{array}$} \\
\cline { 2 - 4 } & $\mathbf{E}$ & $\mathbf{E}$ & $\mathbf{E}_{\text {int }}$ \\
\hline $\mathbf{4 8}$ & -533.494903 & -532.897358 & -5.7 \\
$\mathbf{4 8}$ & -533.493072 & -532.895454 & -4.5 \\
$\mathbf{4 8}$ & -533.493076 & -532.895417 & -4.4
\end{tabular}




\begin{tabular}{|c|c|c|c|}
\hline 49 & -651.190523 & -650.420490 & -7.3 \\
\hline 49 & -651.190091 & -650.419524 & -6.7 \\
\hline 49 & -651.189136 & -650.419219 & -6.5 \\
\hline 49 & -651.189347 & -650.419216 & -6.5 \\
\hline 49 & -651.189198 & -650.419059 & -6.4 \\
\hline 49 & -651.189053 & -650.419014 & -6.3 \\
\hline 49 & -651.189100 & -650.418765 & -6.2 \\
\hline 49 & -651.189059 & -650.418371 & -5.9 \\
\hline 50 & -651.180350 & -650.408376 & -8.3 \\
\hline 50 & -651.179715 & -650.407381 & -7.7 \\
\hline 50 & -651.179070 & -650.406750 & -7.3 \\
\hline 50 & -651.178723 & -650.406615 & -7.2 \\
\hline 50 & -651.178996 & -650.406545 & -7.1 \\
\hline 50 & -651.177826 & -650.405712 & -6.6 \\
\hline 51 & -651.175044 & -650.403003 & -7.4 \\
\hline 51 & -651.174052 & -650.402058 & -6.8 \\
\hline 51 & -651.174055 & -650.402041 & -6.8 \\
\hline 51 & -651.173930 & -650.401920 & -6.7 \\
\hline 51 & -651.173466 & -650.401440 & -6.4 \\
\hline 52 & -994.071831 & -992.895152 & -7.8 \\
\hline 52 & -994.070732 & -992.894167 & -7.2 \\
\hline 52 & -994.070681 & -992.893907 & -7.0 \\
\hline 52 & -994.070737 & -992.893891 & -7.0 \\
\hline 52 & -994.070600 & -992.893731 & -6.9 \\
\hline 52 & -994.070552 & -992.893707 & -6.9 \\
\hline 52 & -994.070278 & -992.893624 & -6.9 \\
\hline 52 & -994.070240 & -992.893431 & -6.7 \\
\hline 52 & -994.070118 & -992.893393 & -6.7 \\
\hline 52 & -994.069972 & -992.893322 & -6.7 \\
\hline 53 & -657.201798 & -656.394481 & -7.3 \\
\hline 53 & -657.201956 & -656.394054 & -7.0 \\
\hline 53 & -657.201157 & -656.393786 & -6.8 \\
\hline 53 & -657.201117 & -656.393622 & -6.7 \\
\hline 53 & -657.200995 & -656.393562 & -6.7 \\
\hline 54 & -689.275233 & -688.458392 & -8.5 \\
\hline 54 & -689.274830 & -688.457851 & -8.2 \\
\hline 54 & -689.274798 & -688.457813 & -8.2 \\
\hline 54 & -689.274236 & -688.457456 & -8.0 \\
\hline
\end{tabular}




\begin{tabular}{|c|c|c|c|}
\hline 54 & -689.273754 & -688.456910 & -7.6 \\
\hline 54 & -689.273419 & -688.456843 & -7.6 \\
\hline 54 & -689.273512 & -688.456153 & -7.1 \\
\hline 54 & -689.273089 & -688.455820 & -6.9 \\
\hline 55 & -705.311354 & -704.490554 & -9.1 \\
\hline 55 & -705.310847 & -704.489754 & -8.5 \\
\hline 55 & -705.310806 & -704.489720 & -8.5 \\
\hline 55 & -705.309763 & -704.489396 & -8.3 \\
\hline 55 & -705.310104 & -704.489300 & -8.3 \\
\hline 55 & -705.309525 & -704.488881 & -8.0 \\
\hline 56 & -748.481563 & -747.603308 & -8.7 \\
\hline 56 & -748.481719 & -747.603209 & -8.7 \\
\hline 56 & -748.481171 & -747.603079 & -8.6 \\
\hline 56 & -748.481059 & -747.602955 & -8.5 \\
\hline 56 & -748.481024 & -747.602864 & -8.5 \\
\hline 56 & -748.480780 & -747.602594 & -8.3 \\
\hline 56 & -748.480848 & -747.602442 & -8.2 \\
\hline 56 & -748.480608 & -747.602361 & -8.2 \\
\hline 56 & -748.480363 & -747.602305 & -8.1 \\
\hline 56 & -748.480546 & -747.602220 & -8.1 \\
\hline 56 & -748.480337 & -747.602044 & -8.0 \\
\hline 56 & -748.479438 & -747.601110 & -7.4 \\
\hline 57 & -768.318126 & -767.433585 & -8.6 \\
\hline 57 & -768.317704 & -767.433363 & -8.5 \\
\hline 57 & -768.316801 & -767.432679 & -8.0 \\
\hline 57 & -768.317124 & -767.432416 & -7.9 \\
\hline 57 & -768.316667 & -767.432372 & -7.9 \\
\hline 57 & -768.316520 & -767.432346 & -7.8 \\
\hline 57 & -768.316720 & -767.432176 & -7.7 \\
\hline 57 & -768.316851 & -767.432043 & -7.7 \\
\hline 57 & -768.316284 & -767.431127 & -7.1 \\
\hline 5 & -764.510985 & -763.628634 & -8.9 \\
\hline 58 & -764.511481 & -763.628627 & -8.9 \\
\hline 58 & -764.510615 & -763.628324 & -8.8 \\
\hline 58 & -764.509842 & -763.627456 & -8.2 \\
\hline 58 & -764.509833 & -763.627402 & -8.2 \\
\hline 58 & -764.509771 & -763.627347 & -8.1 \\
\hline 58 & -764.511424 & -763.627338 & -8.1 \\
\hline 58 & -764.510306 & -763.627047 & -8.0 \\
\hline
\end{tabular}


Table S13. Absolute energies and binding energies for all unique dimers of 47-58 with the model p-methylphenol (Tyr).

\begin{tabular}{|c|c|c|c|}
\hline \multirow[t]{2}{*}{ Het. } & \multirow{2}{*}{$\begin{array}{c}\begin{array}{c}\text { B97D } \\
\text { /def2-TZVPP }\end{array} \\
E\end{array}$} & \multicolumn{2}{|c|}{$\begin{array}{c}\text { DLPNO-CCSD(T) } \\
\text { /cc-pVQZ }\end{array}$} \\
\hline & & $\mathbf{E}$ & $\mathbf{E}_{\text {int }}$ \\
\hline 47 & -608.701923 & -608.040362 & -5.6 \\
\hline 47 & -608.701184 & -608.039560 & -5.1 \\
\hline 47 & -608.700920 & -608.039298 & -4.9 \\
\hline 47 & -608.700428 & -608.038745 & -4.6 \\
\hline 48 & -608.709743 & -608.050496 & -5.7 \\
\hline 48 & -608.709714 & -608.050429 & -5.6 \\
\hline 49 & -726.405720 & -725.574180 & -7.6 \\
\hline 49 & -726.405582 & -725.573919 & -7.5 \\
\hline 49 & -726.404681 & -725.573028 & -6.9 \\
\hline 49 & -726.404272 & -725.572105 & -6.3 \\
\hline 50 & -726.396839 & -725.562490 & -8.9 \\
\hline 50 & -726.395252 & -725.561612 & -8.3 \\
\hline 50 & -726.395057 & -725.561259 & -8.1 \\
\hline 50 & -726.394987 & -725.561242 & -8.1 \\
\hline 50 & -726.395096 & -725.561204 & -8.1 \\
\hline 50 & -726.394716 & -725.560962 & -7.9 \\
\hline 50 & -726.393959 & -725.559965 & -7.3 \\
\hline 50 & -726.393568 & -725.559683 & -7.1 \\
\hline 51 & -726.391488 & -725.557537 & -8.3 \\
\hline 51 & -726.390637 & -725.556867 & -7.9 \\
\hline 51 & -726.390496 & -725.556747 & -7.8 \\
\hline 51 & -726.390262 & -725.556739 & -7.8 \\
\hline 51 & -726.390373 & -725.556735 & -7.8 \\
\hline 51 & -726.390349 & -725.556632 & -7.7 \\
\hline 51 & -726.389335 & -725.555728 & -7.2 \\
\hline 52 & -1069.286982 & -1068.048669 & -8.1 \\
\hline 52 & -1069.286179 & -1068.047987 & -7.7 \\
\hline 52 & -1069.286636 & -1068.047982 & -7.6 \\
\hline 52 & -1069.285949 & -1068.047720 & -7.5 \\
\hline 52 & -1069.286744 & -1068.047615 & -7.4 \\
\hline 52 & -1069.286304 & -1068.047597 & -7.4 \\
\hline 52 & -1069.286118 & -1068.047577 & -7.4 \\
\hline
\end{tabular}




\begin{tabular}{|c|c|c|c|}
\hline 52 & -1069.285697 & -1068.047396 & -7.3 \\
\hline 53 & -732.418162 & -731.548690 & -8.0 \\
\hline 53 & -732.418607 & -731.548581 & -7.9 \\
\hline 53 & -732.417323 & -731.548471 & -7.8 \\
\hline 3 & -732.417988 & -731.548462 & -7.8 \\
\hline 3 & -732.417250 & -731.548362 & -7.8 \\
\hline 53 & -732.416827 & -731.547487 & -7.2 \\
\hline 53 & -732.416425 & -731.547176 & -7.0 \\
\hline 4 & -764.492659 & -763.6 & -9.8 \\
\hline A & -764.491178 & -763.612706 & -9.3 \\
\hline 54 & -764.490890 & -763.612583 & -9.2 \\
\hline & -764.490613 & -763.611943 & -8.8 \\
\hline 54 & -764.489405 & -763.610398 & -7.8 \\
\hline 55 & -780. & -779. & -10.3 \\
\hline 55 & -780.5 & -779.6 & -9.7 \\
\hline 55 & -780.527569 & -779.644527 & -9.6 \\
\hline 5 & -780.526788 & -779.644418 & -9.5 \\
\hline 56 & -823.700188 & -822.7 & -11.3 \\
\hline 6 & -823.7 & -822 & -11.2 \\
\hline 56 & -823.699804 & -822.760033 & -11.0 \\
\hline 56 & -823.698396 & -822.758776 & -10.2 \\
\hline 56 & -823.696876 & -822.757188 & -9.2 \\
\hline 56 & -823.6 & -82 & -9.0 \\
\hline 56 & -823.696567 & -822.756612 & -8.9 \\
\hline 56 & -823.696518 & -822.756593 & -8.8 \\
\hline 56 & -823.696173 & -822.756303 & -8.7 \\
\hline 56 & -823.696249 & -822.756201 & -8.6 \\
\hline 56 & -823.695913 & -822.755901 & -8.4 \\
\hline 57 & -843.533772 & -842.587761 & -9.3 \\
\hline 57 & -843.533512 & -842.587605 & -9.2 \\
\hline 57 & -843.533365 & -842.587253 & -9.0 \\
\hline 57 & -843.533070 & -842.587221 & -8.9 \\
\hline 57 & -843.533392 & -842.586752 & -8.6 \\
\hline 57 & -843.533418 & -842.586691 & -8.6 \\
\hline 57 & -843.533092 & -842.586187 & -8.3 \\
\hline 57 & -843.531606 & -842.585604 & -7.9 \\
\hline 58 & -839.726742 & -838.782719 & -9.6 \\
\hline 58 & -839.725896 & -838.781067 & -8.5 \\
\hline
\end{tabular}




$\mathbf{5 8} \quad-839.724541 \quad-838.780233 \quad-8.0$

Table S14. Absolute energies and binding energies for all unique dimers of 47-58 with the model 3-methylindole (Trp).

\begin{tabular}{|c|c|c|c|}
\hline \multirow[t]{2}{*}{ Het. } & \multirow{2}{*}{$\begin{array}{c}\begin{array}{c}\text { B97D } \\
\text { /def2-TZVPP }\end{array} \\
\mathbf{E} \\
\end{array}$} & \multicolumn{2}{|c|}{$\begin{array}{c}\text { DLPNO-CCSD(T) } \\
\text { /cc-pVQZ }\end{array}$} \\
\hline & & $\mathbf{E}$ & $\mathbf{E}_{\text {int }}$ \\
\hline 47 & -665.012170 & -664.270613 & -7.2 \\
\hline 47 & -665.011309 & -664.270064 & -6.9 \\
\hline 47 & -665.011395 & -664.269892 & -6.7 \\
\hline 47 & -665.011030 & -664.269728 & -6.6 \\
\hline 47 & -665.010672 & -664.268576 & -5.9 \\
\hline 48 & -665.021060 & -664.281932 & -8.0 \\
\hline 48 & -665.019946 & -664.280660 & -7.2 \\
\hline 48 & -665.019494 & -664.280224 & -6.9 \\
\hline 48 & -665.018847 & -664.280086 & -6.9 \\
\hline 49 & -782.716784 & -781.804109 & -9.0 \\
\hline 49 & -782.715723 & -781.803985 & -8.9 \\
\hline 49 & -782.715778 & -781.803891 & -8.9 \\
\hline 49 & -782.715475 & -781.803881 & -8.9 \\
\hline 49 & -782.716054 & -781.803835 & -8.9 \\
\hline 49 & -782.715721 & -781.803724 & -8.8 \\
\hline 49 & -782.715067 & -781.803402 & -8.6 \\
\hline 49 & -782.715488 & -781.803401 & -8.6 \\
\hline 49 & -782.714477 & -781.803309 & -8.5 \\
\hline 49 & -782.714447 & -781.802685 & -8.1 \\
\hline 49 & -782.714893 & -781.802531 & -8.0 \\
\hline 49 & -782.714386 & -781.802255 & -7.9 \\
\hline 49 & -782.713589 & -781.801652 & -7.5 \\
\hline 49 & -782.713001 & -781.801440 & -7.4 \\
\hline 50 & -782.707856 & -781.794096 & -11.3 \\
\hline 50 & -782.707804 & -781.793827 & -11.2 \\
\hline 50 & -782.707509 & -781.793617 & -11.0 \\
\hline 50 & -782.706787 & -781.792638 & -10.4 \\
\hline 50 & -782.706407 & -781.792186 & -10.1 \\
\hline 50 & -782.705434 & -781.792107 & -10.1 \\
\hline 50 & -782.706315 & -781.791707 & -9.8 \\
\hline 50 & -782.705789 & -781.791358 & -9.6 \\
\hline 50 & -782.704746 & -781.790793 & -9.3 \\
\hline
\end{tabular}




\begin{tabular}{llll}
$\mathbf{5 0}$ & -782.704274 & -781.790738 & -9.2 \\
$\mathbf{5 0}$ & -782.704326 & -781.790629 & -9.2 \\
$\mathbf{5 0}$ & -782.704190 & -781.790081 & -8.8 \\
$\mathbf{5 0}$ & -782.704515 & -781.789940 & -8.7 \\
$\mathbf{5 0}$ & -782.703424 & -781.789894 & -8.7 \\
$\mathbf{5 0}$ & -782.702740 & -781.788302 & -7.7 \\
& & & \\
$\mathbf{5 1}$ & -782.703660 & -781.788957 & -10.6 \\
$\mathbf{5 1}$ & -782.703438 & -781.788929 & -10.6 \\
$\mathbf{5 1}$ & -782.700938 & -781.787199 & -9.5 \\
$\mathbf{5 1}$ & -782.700681 & -781.786745 & -9.2 \\
$\mathbf{5 1}$ & -782.700703 & -781.786481 & -9.1 \\
$\mathbf{5 1}$ & -782.699627 & -781.786032 & -8.8 \\
$\mathbf{5 1}$ & -782.699377 & -781.785740 & -8.6 \\
$\mathbf{5 1}$ & -782.699247 & -781.785243 & -8.3 \\
$\mathbf{5 1}$ & -782.698536 & -781.784612 & -7.9 \\
$\mathbf{5 1}$ & -782.698253 & -781.784573 & -7.9 \\
& & & \\
$\mathbf{5 2}$ & -1125.597437 & -1124.279332 & -9.9 \\
$\mathbf{5 2}$ & -1125.597394 & -1124.279232 & -9.9 \\
$\mathbf{5 2}$ & -1125.597330 & -1124.279169 & -9.8 \\
$\mathbf{5 2}$ & -1125.597841 & -1124.279157 & -9.8 \\
$\mathbf{5 2}$ & -1125.596867 & -1124.278921 & -9.7 \\
$\mathbf{5 2}$ & -1125.597886 & -1124.278795 & -9.6 \\
$\mathbf{5 2}$ & -1125.597653 & -1124.278657 & -9.5 \\
$\mathbf{5 2}$ & -1125.596964 & -1124.278534 & -9.4 \\
$\mathbf{5 2}$ & -1125.596539 & -1124.278429 & -9.4 \\
$\mathbf{5 2}$ & -1125.596146 & -1124.278419 & -9.4 \\
$\mathbf{5 2}$ & -1125.596622 & -1124.278332 & -9.3 \\
$\mathbf{5 2}$ & -1125.596511 & -1124.278089 & -9.2 \\
$\mathbf{5 2}$ & -1125.596794 & -1124.278027 & -9.1 \\
$\mathbf{5 2}$ & -1125.596539 & -1124.277990 & -9.1 \\
$\mathbf{5 2}$ & -1125.596438 & -1124.277804 & -9.0 \\
$\mathbf{5 2}$ & -1125.596640 & -1124.277763 & -9.0 \\
$\mathbf{5 2}$ & -1125.595515 & -1124.277595 & -8.8 \\
$\mathbf{5 2}$ & -1125.596237 & -1124.277506 & -8.8 \\
$\mathbf{5 2}$ & -1125.595100 & -1124.277300 & -8.7 \\
$\mathbf{5 2}$ & -1125.595334 & -1124.276994 & -8.5 \\
$\mathbf{5 2}$ & -1125.595135 & -1124.276947 & -8.4 \\
& -1125.595453 & -1124.276830 & -8.4 \\
\hline $\mathbf{5 2}$ & & -1124.276445 & -8.1 \\
\hline
\end{tabular}




\begin{tabular}{|c|c|c|c|}
\hline 3 & 78 & $-7 \varepsilon$ & -9 \\
\hline & 788.727527 & -787.778080 & -9.0 \\
\hline & -788.726358 & -787.777378 & -8.6 \\
\hline & -788.726344 & -787.777319 & -8.5 \\
\hline & -820.802154 & -819.843634 & 11.3 \\
\hline & -820.802294 & 3271 & -11.1 \\
\hline & -820.801894 & -819.843236 & 111 \\
\hline & -820 & -81 & \\
\hline & -820 & 2924 & -10.9 \\
\hline & -820 . & 2628 & -10.7 \\
\hline & -820 & 2443 & -10.6 \\
\hline & 437 & 2416 & -10.6 \\
\hline & -820 & -81 & -10.5 \\
\hline & -820.801099 & 2185 & -10.4 \\
\hline & -820.8 & 973 & -10.3 \\
\hline & -820.8 & 41821 & -10.2 \\
\hline & -820 & 674 & -10.1 \\
\hline & 0727 & -81 & -10.1 \\
\hline & -82 & & -10.0 \\
\hline & -820 & -81 & -9.9 \\
\hline & -820 & -81 & -9.8 \\
\hline & -820 & -81 & -9.8 \\
\hline & -820 & -81 & -9.7 \\
\hline & -820 & -81 & -9.6 \\
\hline 4 & -820 & -81 & -9.4 \\
\hline 54 & -820 & -81 & -9.2 \\
\hline & -820 & -81 & -9.2 \\
\hline & -820 & -81 & -9.1 \\
\hline 4 & -820.798725 & -81 & -9.0 \\
\hline & -820 & -81 & -8.7 \\
\hline & -820.797082 & -819.838368 & -8.0 \\
\hline & -836.8 & -835 & -11.5 \\
\hline & -836 & 5062 & 1.4 \\
\hline & -836 & 928 & -11.3 \\
\hline & -836 & -83 & -11.3 \\
\hline & -836.837833 & 74816 & -11.2 \\
\hline & -836.837546 & & \\
\hline & -836.837169 & 4616 & -11.1 \\
\hline & -836.837679 & -835.874536 & -11.0 \\
\hline 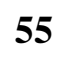 & -836.837416 & -835.874473 & -11.0 \\
\hline 55 & -836.8 & -835.874324 & 10.9 \\
\hline 55 & -836.837481 & -835.874307 & -10.9 \\
\hline
\end{tabular}




\begin{tabular}{|c|c|c|c|}
\hline & 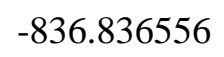 & 72 & -1 \\
\hline & 336.836943 & -835.874135 & -10.8 \\
\hline & 836.836483 & -835.874032 & -10.7 \\
\hline & 836.836268 & 835.873997 & -10.7 \\
\hline & 6259 & 68 & -10.6 \\
\hline & 6348 & 3814 & -10.6 \\
\hline & 6420 & 3756 & -10.5 \\
\hline & -836.835728 & & -10.4 \\
\hline & 051 & 58 & -10.4 \\
\hline & 202 & 129 & -10.3 \\
\hline & -836 & -835 & -10.3 \\
\hline & -836 & & -10.0 \\
\hline & 691 & 01 & -9.9 \\
\hline & -836 & & -9.7 \\
\hline & 10 & 10 & -8.9 \\
\hline & 880 & 78 & -11.9 \\
\hline & -880 & & -11.6 \\
\hline & 769 & 81 & -11.5 \\
\hline & 1 & & -11.4 \\
\hline & 731 & 216 & -10.7 \\
\hline & -88 & 148 & -10.6 \\
\hline & 318 & 04 & -10.5 \\
\hline & & & -10.4 \\
\hline & -880 & -87 & -10.4 \\
\hline & -88 & -87 & -10.4 \\
\hline & 670 & & -10.2 \\
\hline & & & -10.2 \\
\hline & -880 & & -10.2 \\
\hline & -880 & -87 & -9.7 \\
\hline 56 & -880.004922 & -878 & -9.1 \\
\hline & . & 76 & -11.9 \\
\hline & -899.8 & -898 & -11.9 \\
\hline 7 & -899.846329 & -898 & -11.6 \\
\hline & & -898 & -11.1 \\
\hline & -899. & -898 & -11.0 \\
\hline 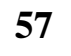 & -899.845507 & -898.8 & -11.0 \\
\hline 57 & -899.8 & -898 & -11.0 \\
\hline . & -899.845035 & -898.818032 & -10.9 \\
\hline & -899.844404 & -898 & -10.9 \\
\hline 57 & -899.843289 & -898.817297 & -10.4 \\
\hline 57 & -899.843355 & -898.817296 & -10.4 \\
\hline 5 & -899.843202 & -898.816919 & -10.2 \\
\hline
\end{tabular}




\begin{tabular}{|c|c|c|c|}
\hline 57 & -899.842346 & -898.816758 & -10.1 \\
\hline 57 & -899.842649 & -898.816600 & -10.0 \\
\hline 57 & -899.841710 & -898.816053 & -9.7 \\
\hline 57 & -899.842355 & -898.815997 & -9.6 \\
\hline 57 & -899.841458 & -898.815800 & -9.5 \\
\hline 57 & -899.841444 & -898.815540 & -9.3 \\
\hline 57 & -899.841340 & -898.815389 & -9.2 \\
\hline 57 & -899.841110 & -898.814299 & -8.5 \\
\hline 57 & -899.839612 & -898.813858 & -8.3 \\
\hline 58 & -896.040917 & -895.016176 & -13.2 \\
\hline 58 & -896.040811 & -895.015732 & -12.9 \\
\hline 58 & -896.040670 & -895.015501 & -12.7 \\
\hline 58 & -896.039847 & -895.014694 & -12.2 \\
\hline 58 & -896.039605 & -895.014130 & -11.9 \\
\hline 58 & -896.039012 & -895.013419 & -11.4 \\
\hline 58 & -896.037961 & -895.013346 & -11.4 \\
\hline 58 & -896.037359 & -895.013027 & -11.2 \\
\hline 58 & -896.036888 & -895.012674 & -11.0 \\
\hline 58 & -896.037124 & -895.012659 & -11.0 \\
\hline 58 & -896.037914 & -895.012576 & -10.9 \\
\hline 58 & -896.036093 & -895.012052 & -10.6 \\
\hline 58 & -896.035369 & -895.010962 & -9.9 \\
\hline 58 & -896.035598 & -895.010921 & -9.9 \\
\hline 58 & -896.034067 & -895.009635 & -9.1 \\
\hline
\end{tabular}

Cartesian coordinates are available as separate XYZ files. 